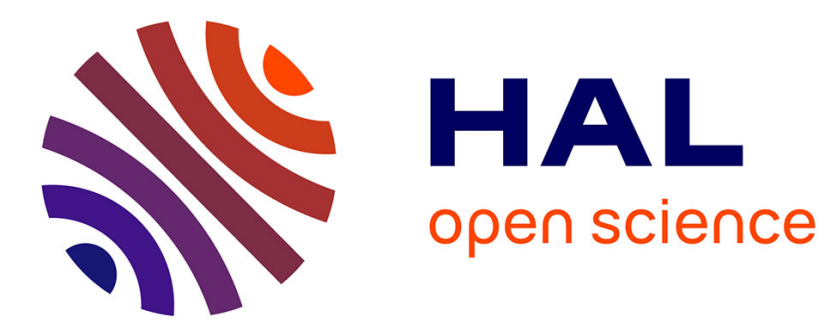

\title{
Linear Periods of Automorphic Sheaves for GL2n
}

Sergey Lysenko

\section{To cite this version:}

Sergey Lysenko. Linear Periods of Automorphic Sheaves for GL2n. International Mathematics Research Notices, 2021, 10.1093/imrn/rnab077 . hal-03281584

\section{HAL Id: hal-03281584 \\ https://hal.univ-lorraine.fr/hal-03281584}

Submitted on 8 Jul 2021

HAL is a multi-disciplinary open access archive for the deposit and dissemination of scientific research documents, whether they are published or not. The documents may come from teaching and research institutions in France or abroad, or from public or private research centers.
L'archive ouverte pluridisciplinaire HAL, est destinée au dépôt et à la diffusion de documents scientifiques de niveau recherche, publiés ou non, émanant des établissements d'enseignement et de recherche français ou étrangers, des laboratoires publics ou privés. 


\section{Linear Periods of Automorphic Sheaves for $\mathrm{GL}_{2 n}$}

\section{S. Lysenko*}

Institut Élie Cartan Nancy (Mathématiques), Université de Lorraine, B.P. 239, F-54506 Vandoeuvre-lès-Nancy Cedex, France

*Correspondence to be sent to: e-mail: Sergey.Lysenko@univ-lorraine.fr

We calculate, in the framework of the geometric Langlands program, the periods of cuspidal automorphic sheaves for $\mathrm{GL}_{2 n}$ along the Levi subgroup $\mathrm{GL}_{n} \times \mathrm{GL}_{n}$. We also solve the corresponding local problem.

\section{Introduction}

1.0 .1

Let $X$ be a smooth projective connected curve over an algebraically closed field $k$. Write Bun $_{n}$ for the moduli stack of rank $n$ vector bundles on $X$. In this paper, we calculate the geometric periods of cuspidal automorphic sheaves on $\mathrm{Bun}_{2 n}$ for the Levi subgroup $\mathrm{GL}_{n} \times \mathrm{GL}_{n}$. Our results geometrize a similar calculation at the level of functions from [4], but our proof does not follow any existing argument of the theory of automorphic forms.

More generally, if $G$ is a connected reductive group over $k, H \subset G$ is a spherical subgroup, one may ask, in the framework of the geometric Langlands program, about the periods of automorphic sheaves on $\operatorname{Bun}_{G}$ with respect to the subgroup $H$. The corresponding problem at the level of functions has been intensively studied in the theory of automorphic forms ([4, 7, 8, 18] and more references in [18]).

In the case $G=\mathrm{GL}_{n} \times \mathrm{GL}_{n}, H$ is the diagonally embedded $\mathrm{GL}_{n}$ this problem was solved in [11, 12], and in loc.cit. It was naturally divided into local and global parts. 
In our case $G=\mathrm{GL}_{2 n}$ and $H$ is the Levi subgroup $\mathrm{GL}_{n} \times \mathrm{GL}_{n}$, it is spherical. We divide this problem into local and global parts also.

If $E$ is an irreducible rank $2 n$ local system on $X$, one has the automorphic $E$ Hecke eigensheaf $\mathrm{Aut}_{E}$ on $\mathrm{Bun}_{2 n}$ constructed in $[2,5]$. The linear periods of Aut $E$ along $\mathrm{GL}_{n} \times \mathrm{GL}_{n}$ allow to distinguish the local systems $E$ on $X$, which admit a symplectic form (so, conjecturally, then $\mathrm{Aut}_{E}$ comes by the geometric Langlands functoriality from a smaller group).

This paper is also motivated by [14], where for $G=G S p_{4}$ and a $\breve{G}$-local system $E_{\breve{G}}$ on $X$ whose standard representation is irreducible, we constructed a $E_{\breve{G}}$-Hecke eigensheaf $\mathcal{K}_{E_{\breve{G}}}$ on $\mathrm{Bun}_{G}$. In [14], we have not checked that $\mathcal{K}_{E_{\breve{G}}}$ is always nonzero. This nonvanishing is established in this paper.

\subsection{2}

In this section, we informally describe our main results and the ideas involved in the proofs.

Write Pic $X$ for the Picard stack of $X$. Consider the diagram

$$
\operatorname{Bun}_{2 n} \stackrel{\nu_{n}}{\leftarrow} \operatorname{Bun}_{n} \times \operatorname{Bun}_{n} \stackrel{\operatorname{det} \times \operatorname{det}}{\rightarrow} \operatorname{Pic} X \times \operatorname{Pic} X,
$$

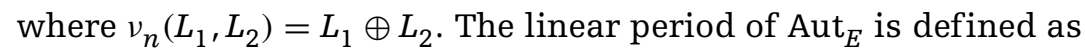

$$
\operatorname{Per}_{E}=(\operatorname{det} \times \operatorname{det}) ! v_{n}^{*} \operatorname{Aut}_{E}\left[\operatorname{dim} \cdot \operatorname{rel}\left(v_{n}\right)\right]
$$

For $d \geq 0$, let $X^{(d)}$ denote the $d$ th symmetric power of $X$. Write $\Omega$ for the canonical line bundle on $X$. Let $\epsilon_{X}: X^{(d)} \times X^{\left(d^{\prime}-d\right)} \rightarrow \operatorname{Pic} X \times \operatorname{Pic} X$ be the map sending $\left(D, D_{1}\right)$ to

$$
\left(\Omega^{(2 n-1)+(2 n-3)+\ldots+1}(D), \Omega^{(2 n-2)+(2 n-4)+\ldots+2}\left(D+D_{1}\right)\right)
$$

For $d, d^{\prime} \in \mathbb{Z}$, let $(\operatorname{Pic} X \times \operatorname{Pic} X)^{d, d^{\prime}}$ be the connected component of $\operatorname{Pic} X \times \operatorname{Pic} X$ given for $\left(\mathcal{A}, \mathcal{A}^{\prime}\right) \in \operatorname{Pic} X \times \operatorname{Pic} X$ by

$$
\operatorname{deg} \mathcal{A}=d+\operatorname{deg}\left(\Omega^{(2 n-1)+(2 n-3)+\ldots+1}\right), \quad \operatorname{deg} \mathcal{A}^{\prime}=d^{\prime}+\operatorname{deg}\left(\Omega^{(2 n-2)+(2 n-4)+\ldots+2}\right)
$$

Our main global result is Theorem 2.2.2, Theorem which describes all the left truncations $\tau_{\geq N} \operatorname{Per}_{E}$ for $N \in \mathbb{Z}$ over $(\operatorname{Pic} X \times \operatorname{Pic} X)^{d, d^{\prime}}$. The answer is of local nature with respect to $X$, it makes sense for any, not necessarily irreducible, local system $E$ on $X$. 
The answer is given essentially by a suitable left truncation of

$$
\left(\epsilon_{X}\right)_{!}\left(\left(\wedge^{2} E\right)^{(d)} \otimes E^{\left(d^{\prime}-d\right)}\right)
$$

(cf. Theorem 2.2.2 for a precise claim). This is an easy consequence of Theorem 2.1.2, which is our main local result.

Let $\mathcal{M}_{n}$ be the stack classifying $L \in \operatorname{Bun}_{n}$ with a nonzero section $\Omega^{n-1} \hookrightarrow L$, $q_{n}: \mathcal{M}_{n} \rightarrow \operatorname{Bun}_{n}$ be the map sending this point to $L$. Our formulation of Theorem 2.1.2 uses special features of the $\mathrm{GL}_{n}$ case, the existence of the mirabolic subgroup used in Laumon's construction of automorphic sheaves on $\operatorname{Bun}_{n}$ via descent under $\mathcal{M}_{n} \rightarrow \operatorname{Bun}_{n}$. Namely, we replace (1) by the diagram

$$
\mathcal{M}_{2 n} \stackrel{\nu_{\mathcal{Z}}}{\leftarrow} \mathcal{Z}_{n} \stackrel{\pi}{\rightarrow} \operatorname{Pic} X \times \operatorname{Pic} X,
$$

where $\mathcal{Z}_{n}$ is the stack classifying $L, L^{\prime} \in \operatorname{Bun}_{n}$ with a nonzero section $\Omega^{2 n-1} \hookrightarrow L$. The map $v_{\mathcal{Z}}$ sends this point to $M=L \oplus L^{\prime}$ with the induced section $\Omega^{2 n-1} \hookrightarrow M$, and $\pi$ sends the above point to $\left(\operatorname{det} L, \operatorname{det} L^{\prime}\right)$.

Write $\mathcal{M}_{n, d}$ for the connected component of $\mathcal{M}_{n}$ given by

$$
\operatorname{deg} L-\operatorname{deg}\left(\Omega^{(n-1)+(n-2)+\ldots+1}\right)=d
$$

To a local system $E$ on $X$, Laumon has associated a complex $\mathcal{K}_{n, E}^{d}$ on $\mathcal{M}_{n, d}$ for $d \geq 0$ (cf. Section 2.3.1). If $E$ is irreducible of rank $n$, then one has $q_{n}^{*} \operatorname{Aut}_{E}\left[\operatorname{dim} \cdot \operatorname{rel}\left(q_{n}\right)\right] \widetilde{\mathcal{K}_{n, E}^{d}}$ for $d \geq 0$ over $\mathcal{M}_{n, d}$ by $[2,5]$.

We define the local linear period of $\mathcal{K}_{2 n, E}$ as

$$
\pi_{!}\left(v_{\mathcal{Z}}^{*} \mathcal{K}_{2 n, E}\right)\left[\operatorname{dim} . \operatorname{rel}\left(v_{\mathcal{Z}}\right)\right]
$$

Theorem 2.1.2 claims that for $d+d^{\prime} \geq 0$ and any local system $E$ on $X$ of rank $2 n$ the restriction of (3) to $(\operatorname{Pic} X \times \operatorname{Pic} X)^{d, d^{\prime}}$ vanishes unless $0 \leq d \leq d^{\prime}$, and in the latter case (assuming for simplicity $\mathrm{rk} E=2 n$ ) identifies with (2) up to a shift.

In Section 2.3, we explain our plan of the proof of Theorem 2.1.2 reducing it to Propositions 2.3.4 and 2.3.5.

For $d \geq 0$ in Section 2.3.1, we define the morphism $q_{\mathcal{Y}}: \mathcal{Y}_{n, d} \rightarrow \mathcal{M}_{n, d}$ and a perverse sheaf $\mathcal{P}_{n, E}^{d}$ on $\mathcal{Y}_{n, d}$ appearing in Laumon's construction. By definition, if $E$ is a local system on $X$, then $\mathcal{K}_{n, E}^{d} \widetilde{\rightarrow}\left(q_{\mathcal{Y}}\right)_{!} \mathcal{P}_{n, E}^{d}$. So, Theorem 2.1 .2 calculates certain direct 
image under the composition

$$
\mathcal{Y}_{2 n} \times \mathcal{M}_{2 n} \mathcal{Z}_{n} \stackrel{\mathrm{pr}_{2}}{\rightarrow} \mathcal{Z}_{n} \stackrel{\pi}{\rightarrow} \operatorname{Pic} X \times \operatorname{Pic} X
$$

In Section 2.3.3, we get a commutative diagram

$$
\begin{array}{ccccc}
\widetilde{\mathcal{Y}}_{n, d} \times \widetilde{\mathcal{Y}}_{n, d^{\prime}}^{\prime} & \stackrel{\bar{\pi}}{\rightarrow} & X^{(d)} \times X^{\left(d^{\prime}\right)} & \stackrel{i_{X}}{\leftarrow} X^{(d)} \times X^{\left(d^{\prime}-d\right)} \\
\downarrow q \mathcal{Y Z} & & \downarrow \epsilon & \swarrow \epsilon_{X} \\
\mathcal{Y}_{2 n} \times \mathcal{M}_{2 n} \mathcal{Z}_{2 n} & \stackrel{\pi \circ \mathrm{pr}_{2}}{\rightarrow} & \operatorname{Pic} X \times \operatorname{Pic} X, & &
\end{array}
$$

where $q_{\mathcal{Y Z}}$ is a closed immersion. Here $i_{X}$ sends $\left(D, D_{1}\right)$ to $\left(D, D+D_{1}\right)$. Our Proposition 2.3.4 close in spirit to ([11], Theorem A) allows to replace the integration over $\mathcal{Y}_{2 n} \times \mathcal{M}_{2 n} \mathcal{Z}_{n}$ by the integration over its closed substack $\widetilde{\mathcal{Y}}_{n, d} \times \widetilde{\mathcal{Y}}_{n, d^{\prime}}^{\prime}$. Theorem 2.1.2 is so reduced to a calculation of the direct image

$$
\bar{\pi}_{!} q_{\mathcal{Y} \mathcal{Z}}^{*}\left(\mathcal{P}_{2 n, E} \otimes \overline{\mathbb{Q}}_{\ell}\right)
$$

in Proposition 2.3.5. Using the natural stratification of $\mathcal{Y}_{2 n}$ from [11] and the description of the $*$-restrictions of $\mathcal{P}_{2 n, E}$ to the strata obtained in loc.cit., we first calculate (4) via this stratification. This shows that (4) is placed in one cohomological degree and provides a filtration on the corresponding constructible sheaf. Besides, we see that this sheaf is the extension by zero under $i_{X}$. It remains to identify this constructible sheaf with $\left(i_{X}\right)_{!}\left(\wedge^{2} E\right)^{(d)} \otimes E^{\left(d^{\prime}-d\right)}$ (in the case $\operatorname{rk}(E)=2 n$ ).

The latter problem is absent at the level of functions. At the geometric level, on the contrary, this remaining part is the most difficult and involves new ideas. We divide it into two steps:

STEP (1) is Proposition 4.2.4. Unwinding Laumon's construction of $\mathcal{P}_{2 n, E}$, we see that the problem is to calculate certain highest direct image with compact support (for the usual t-structure). We introduce a diagram

$$
\widetilde{\mathcal{Q}}_{n, d} \times \widetilde{\mathcal{Q}}_{n, d^{\prime}}^{\prime} \stackrel{\tilde{\varphi} \times \tilde{\varphi}^{\prime}}{\rightarrow} \widetilde{\mathcal{Y}}_{n, d} \times \widetilde{\mathcal{Y}}_{n, d^{\prime}}^{\prime} \stackrel{\bar{\pi}}{\rightarrow} X^{(d)} \times X^{\left(d^{\prime}\right)}
$$

and identify the cohomology sheaf of (4) in the corresponding degree with some highest direct image under $\bar{\pi} \circ\left(\tilde{\varphi} \times \tilde{\varphi}^{\prime}\right)$. This is obtained by a reduction to some local statement with respect to $X$. We generalize this local result for an arbitrary reductive group and a Levi subgroup that we call antistandard. This generalization is performed in Appendix A independent of the rest of the paper. We consider this generalization as an important result of independent interest (Propositions A.0.5 and A.2.2). 
STEP (2) is Proposition 4.3.2, which is in turn reduced to Theorem 4.3.9. Their content is to decompose $\bar{\pi} \circ\left(\tilde{\varphi} \times \tilde{\varphi}^{\prime}\right)$ as

$$
\widetilde{\mathcal{Q}}_{n, d} \times \widetilde{\mathcal{Q}}_{n, d^{\prime}}^{\prime} \stackrel{\tilde{\beta} \times \tilde{\beta}^{\prime}}{\rightarrow} \leq n \operatorname{Sh}_{0}^{d} \times \leq n \operatorname{Sh}_{0}^{d^{\prime}} \stackrel{\leq n}{\stackrel{\operatorname{div} \times \operatorname{div})}{\rightarrow}} X^{(d)} \times X^{\left(d^{\prime}\right)}
$$

and reduce the calculation of the highest direct image under $\bar{\pi} \circ\left(\tilde{\varphi} \times \tilde{\varphi}^{\prime}\right)$ to a calculation of the highest direct image under ${ }^{\leq n}(\operatorname{div} \times \operatorname{div})$ over the closed subscheme.

$$
i_{X}: X^{(d)} \times X^{\left(d^{\prime}-d\right)} \hookrightarrow X^{(d)} \times X^{\left(d^{\prime}\right)}
$$

The last calculation given in Theorem 4.3.9 is of local nature with respect to $X$. We consider Theorem 4.3.9 as one of our main results, which is of independent interest.

For $0 \leq d \leq d^{\prime}$, in Section 4.3.5, we introduce the scheme $V_{-}^{d, d^{\prime}}$ classifying $D \in X^{(d)}, D^{\prime} \in X^{\left(d^{\prime}\right)}$ with $D \leq D^{\prime}$ and $\left(x_{i}\right) \in X^{d+d^{\prime}}$ with $\sum_{i} x_{i}=D+D^{\prime}$. The IC-sheaf of $V_{-}^{d, d^{\prime}}$ is described in Section 4.3.6, which is a constructible sheaf placed in usual cohomological degree $-d^{\prime}$. Let $\mathrm{Sh}_{-}^{d, d^{\prime}}$ be the stack classifying torsion sheaves $F, F^{\prime}$ on $X$ with $\operatorname{div}(F) \leq \operatorname{div}\left(F^{\prime}\right), d=\operatorname{deg} F, d^{\prime}=\operatorname{deg} F^{\prime}$, and a complete flag of torsion sheaves $F_{1} \subset \ldots \subset F_{d+d^{\prime}}=F \oplus F^{\prime}$. We have the map $\operatorname{div}_{-}^{v}: \mathrm{Sh}_{-}^{d, d^{\prime}} \rightarrow V_{-}^{d, d^{\prime}}$ defined in Section 4.3.8. Theorem 4.3.9 claims, in particular, that each fibre of $\operatorname{div}_{-}^{v}$ is nonempty of dimension $-d^{\prime}$, and

$$
\mathrm{R}^{-2 d^{\prime}}\left(\operatorname{div}_{-}^{v}\right), \overline{\mathbb{Q}}_{\ell} \stackrel{\sim}{\rightarrow} \mathrm{IC}\left[-d^{\prime}\right]
$$

naturally. To prove Theorem 4.3.9, we use two different stratifications of $\mathrm{Sh}_{-}^{d, d^{\prime}}$. The definition of the second stratification is inspired by the results of Richardson and Springer from [17]. Write $\mathcal{E}$ for the set of irreducible components of $V_{-}^{d, d^{\prime}}$. We get an inclusion of $\mathcal{E}$ in the set indexing the strata of $\mathrm{Sh}_{-}^{d, d^{\prime}}$. It turns out that only these strata contribute to the highest direct image under $\operatorname{div}_{-}^{v}$, and this is the contribution of the corresponding irreducible components of $V_{-}^{d, d^{\prime}}$ into IC[- $\left.d^{\prime}\right]$. We finish the proof of Theorem 4.3.9 using general Remarks 4.4.13 and 2.4.6. This completes the proof of Theorem 2.1.2.

\subsubsection{Organization}

Our main results are formulated in Section 2. In Section 2.4, we state some additional results (one may think of Proposition 2.4.4 as a version of Pieri's rule for $\mathrm{GL}_{m}$ over some configuration spaces of coweights). In Section 2.5, we derive some consequences from Theorem 2.1.2 for the classical theory of automorphic forms. We obtain an integral 
representation of the $L$-function attached to $\wedge^{2} E$ similar to the one given by Friedberg and Jacquet [4]. The proofs are given in the remaining sections.

\subsection{Notation}

Work over an algebraically closed ground field $k$ of characteristic $p>0$ (except in Section 2.5.3, where $k=\mathbb{F}_{q}$ of characteristic $p>0$ ). All the schemes and stacks we consider are defined over $k$. Fix a prime $\ell$ and an algebraic closure $\overline{\mathbb{Q}}_{\ell}$ of $\mathbb{Q}_{\ell}$. Let $X$ be a smooth projective connected curve of genus $g$. Write $\Omega$ for the canonical line bundles on $X$.

We work with algebraic stacks in smooth topology and étale $\overline{\mathbb{Q}}_{\ell}$-sheaves on them. We adopt the conventions of ([13], Section 2.1). So, for an algebraic stack $S$ locally of finite type, we have the derived category $\mathrm{D}(S)$ of $\ell$-adic sheaves on $S$. For a morphism $f: \mathcal{X} \rightarrow \mathcal{Y}$ of algebraic stacks, the functors $f^{*}, f_{*}, f_{!}$between the corresponding derived categories are understood in the derived sense. We use the notion of a generalized affine fibration from ([11], Section 0.1.1). For a morphism of stacks $f: \mathcal{X} \rightarrow \mathcal{Y}$ write $\operatorname{dim}$. $\operatorname{rel}(f)$ for the function of a connected component $C$ of $\mathcal{X}$ given by $\operatorname{dim} C-\operatorname{dim} C^{\prime}$, where $C^{\prime}$ is the connected component of $\mathcal{Y}$ containing $f(C)$.

Fix a nontrivial character $\psi: \mathbb{F}_{p} \rightarrow \overline{\mathbb{Q}}_{\ell}^{*}$ and denote by $\mathcal{L}_{\psi}$, the Artin-Schreier sheaf on $\mathbb{A}^{1}$ associated to $\psi$. We will ignore the Tate twists everywhere (they are easy to recover if necessary).

For $n \geq 1$, write $\operatorname{Bun}_{n}$ for the stack of rank $n$ vector bundles on $X$. For $n=1$, we also write Pic $X=\operatorname{Bun}_{1}$ for the Picard stack of $X$. By a modification $M^{\prime} \subset M^{\prime \prime}$ of a locally free $\mathcal{O}_{X}$-module $M^{\prime \prime}$ on $X$, we mean a quasi-coherent subsheaf such that $M^{\prime}=M^{\prime \prime}$ over the generic point of $X$. For $d \geq 0$ denote by $\mathrm{Sh}_{0}^{d}$, the stack of torsion sheaves on $X$ of generic rank zero and length $d$. Let ${ }^{\leq n} \mathrm{Sh}_{0}^{d} \subset \mathrm{Sh}_{0}^{d}$ be the open substack given by the property: for a scheme $S$ an object $F$ of $\operatorname{Hom}\left(S, \operatorname{Sh}_{0}^{d}\right)$ lies in $\operatorname{Hom}\left(S,{ }^{n} \operatorname{Sh}_{0}^{d}\right)$ if the geometric fibre of $F$ at any point of $S \times X$ is of dimension $\leq n$.

For $d \geq 0$ write $X^{(d)}$ for the $d$-th symmetric power of $X$. We think of it as the scheme of effective divisors of degree $d$ on $X$. Let $\operatorname{div}: \mathrm{Sh}_{0}^{d} \rightarrow X^{(d)}$ be the morphism norm as in ([11], Section 0.1.2).

\section{1 .1}

Fix the maximal torus of diagonal matrices in $\mathrm{GL}_{n}$ and the Borel subgroup of upper triangular matrices. The set of weights of $\mathrm{GL}_{n}$ is identified with $\mathbb{Z}^{n}$. 
We use the following (nonstandard) notations. Let $\Lambda_{m}=\mathbb{Z}^{m}$. For $a, b \in \mathbb{Z}^{m}$, we write $\langle a, b\rangle=\sum_{i} a_{i} b_{i}$. Let $\Lambda_{m}^{+}=\left\{\left(\lambda_{1}, \ldots \lambda_{m}\right) \in \mathbb{Z}^{m} \mid \lambda_{1} \geq \ldots \geq \lambda_{m}\right\}, \Lambda_{m}^{-}=-\Lambda_{m}^{+}$. Let

$$
\Lambda_{m}^{\succ}=\left\{\lambda \in \mathbb{Z}^{m} \mid \lambda_{i} \geq 0 \text { for all } i\right\}
$$

Let $\Lambda_{m}^{\succ+}=\Lambda_{m}^{+} \cap \Lambda_{m}^{\succ}$. Let $\Lambda_{m}^{\succ-}=\Lambda_{m}^{-} \cap \Lambda_{m}^{\succ}$. For $d \in \mathbb{Z}$ let

$$
\Lambda_{m, d}^{\succ+}=\left\{\lambda \in \Lambda_{m}^{\succ+} \mid \sum_{i} \lambda_{i}=d\right\}
$$

The second subindex $d$ on the right will mean that we impose the above condition $\sum_{i} \lambda_{i}=d$. This way we get, in particular, $\Lambda_{m, d}, \Lambda_{m, d}^{\succ-}$ and $\Lambda_{m, d}^{\succ}$.

Let

$$
\Lambda_{m}^{p o s}=\left\{\lambda \in \Lambda_{m, 0} \mid \lambda_{1}+\ldots+\lambda_{i} \geq 0 \text { for } 1 \leq i \leq m\right\}
$$

Set $\Lambda_{m}^{\succ \text { pos }}=\Lambda_{m}^{\succ}+\Lambda_{m}^{\text {pos }}$, we also get $\Lambda_{m, d}^{\succ \text { pos }}=\Lambda_{m, d}^{\succ}+\Lambda_{m}^{\text {pos }}$.

1.1 .2

For $\lambda \in \Lambda_{m}^{\succ \text { pos }}$ set

$$
X_{\text {pos }}^{\lambda}=\prod_{i=1}^{m} X^{\left(\lambda_{1}+\ldots+\lambda_{i}\right)}
$$

This is the scheme of $\Lambda_{m}^{\succ \text { pos }}$-valued divisors of degree $\lambda$. A point of $X_{\text {pos }}^{\lambda}$ is a collection $\left(D_{1}, \ldots, D_{m}\right)$ with $D_{1}+\ldots+D_{i} \in X^{\left(\lambda_{1}+\ldots+\lambda_{i}\right)}$ for $1 \leq i \leq m$.

Let $X^{\lambda} \subset X_{\text {pos }}^{\lambda}$ be the closed subscheme given by the property that $D_{i} \geq 0$ for all $i$. This is the scheme of $\Lambda_{m}^{\succ}$-valued divisors of degree $\lambda$.

Let $X_{-}^{\lambda} \subset X^{\lambda}$ be the closed subscheme given by $D_{1} \leq \ldots \leq D_{m}$. Let also $X_{+}^{\lambda} \subset X^{\lambda}$ be the closed subscheme given by $D_{1} \geq \ldots \geq D_{m}$.

For a local system $E$ on $X$ and $\lambda \in \Lambda_{n}^{\succ-}$ the sheaf $E_{-}^{\lambda}$ on $X_{-}^{\lambda}$ is introduced in ([11], Definition 1). We denote also by $\mathcal{L}_{E}^{d}$ (resp., $\mathcal{S} p r_{E}^{d}$ ) Laumon's (resp., Springer's) perverse sheaf on $\operatorname{Sh}_{0}^{d}$ defined in ([11], Section 1). The group $S_{d}$ acts on $\mathcal{S p r}_{E}^{d}$, and $\mathcal{L}_{E}^{d}$ is the perverse sheaf of $S_{d}$-invariants of $\mathcal{S p r}_{E}^{d}$. 


\section{Main Results}

\section{1}

Fix $n>0$. For $d \in \mathbb{Z}$ as in ([11], Section 2.1), write $\mathcal{M}_{n}$ for the stack classifying $L \in \operatorname{Bun}_{n}$ together with a subsheaf $\Omega^{n-1} \hookrightarrow L$. Write $\mathcal{M}_{n, d}$ for the connected component of $\mathcal{M}_{n}$ given by $\operatorname{deg} L-\operatorname{deg}\left(\Omega^{(n-1)+(n-2)+\ldots+1}\right)=d$. Let $q_{n}: \mathcal{M}_{n, d} \rightarrow \operatorname{Bun}_{n}$ be the projection sending the above point to $L$.

For a local system $E$ on $X$ and $d \geq 0$ one has a complex $\mathcal{K}_{n, E}^{d}$ over $\mathcal{M}_{n, d}$ defined in ([11], Remark 1). If $E$ is irreducible of rank $n$ then for $d \geq 0$ there is an isomorphism $q_{n}^{*}$ Aut $_{E}\left[\operatorname{dim} . \operatorname{rel}\left(q_{n}\right)\right] \underset{\sim}{\rightarrow} \mathcal{K}_{n, E}^{d}$ over $\mathcal{M}_{n, d}$ established in $[2,5]$. Here Aut As is the $E$-Hecke eigensheaf on $\operatorname{Bun}_{n}$ defined in [2], which is perverse. Though $\mathcal{K}_{n, E}$ may depend on $\psi$, we do not express this in our notation.

\subsection{1}

Let $\mathcal{Z}_{n}$ be the stack classifying $L, L^{\prime} \in \operatorname{Bun}_{n}$ and a nonzero section $s: \Omega^{2 n-1} \hookrightarrow L$. Let $v_{\mathcal{Z}}: \mathcal{Z}_{n} \rightarrow \mathcal{M}_{2 n}$ be the map sending this point to $M=L \oplus L^{\prime}$ with the induced section $s: \Omega^{2 n-1} \hookrightarrow M$. We get the diagram

$$
\mathcal{M}_{2 n} \stackrel{\nu_{\mathcal{Z}}}{\leftarrow} \mathcal{Z}_{n} \stackrel{\pi}{\rightarrow} \operatorname{Pic} X \times \operatorname{Pic} X
$$

where $\pi$ is the map sending $\left(L, L^{\prime}, s\right)$ to $\left(\operatorname{det} L, \operatorname{det} L^{\prime}\right)$. Let $E$ be a local system on $X$. The local linear period of $\mathcal{K}_{2 n, E}$ is

$$
\pi_{!}\left(v_{\mathcal{Z}}^{*} \mathcal{K}_{2 n, E}\right)\left[\operatorname{dim} \cdot \operatorname{rel}\left(v_{\mathcal{Z}}\right)\right]
$$

For $d, d^{\prime} \geq 0$ write $\epsilon: X^{(d)} \times X^{\left(d^{\prime}\right)} \rightarrow \operatorname{Pic} X \times \operatorname{Pic} X$ for the map sending $\left(D, D^{\prime}\right)$ to

$$
\left(\Omega^{(2 n-1)+(2 n-3)+\ldots+1}(D), \Omega^{(2 n-2)+(2 n-4)+\ldots+2}\left(D^{\prime}\right)\right)
$$

For $d, d^{\prime} \in \mathbb{Z}$ write $(\operatorname{Pic} X \times \operatorname{Pic} X)^{d, d^{\prime}}$ for the connected component of Pic $X \times \operatorname{Pic} X$ given for $\left(\mathcal{A}, \mathcal{A}^{\prime}\right) \in \operatorname{Pic} X \times \operatorname{Pic} X$ by

$$
\operatorname{deg} \mathcal{A}=d+\operatorname{deg}\left(\Omega^{(2 n-1)+(2 n-3)+\ldots+1}\right), \quad \operatorname{deg} \mathcal{A}^{\prime}=d^{\prime}+\operatorname{deg}\left(\Omega^{(2 n-2)+(2 n-4)+\ldots+2}\right)
$$

If $0 \leq d \leq d^{\prime}$ we have the closed immersion

$$
i_{X}: X^{(d)} \times X^{\left(d^{\prime}-d\right)} \rightarrow X^{(d)} \times X^{\left(d^{\prime}\right)}
$$


sending $\left(D, D_{1}\right)$ to $\left(D, D+D_{1}\right)$. Our main result is the following.

Theorem 2.1.2. Let $E$ be any local system on $X$ and $d+d^{\prime} \geq 0$. Over $(\operatorname{Pic} X \times \operatorname{Pic} X)^{d, d^{\prime}}$ the complex (5) vanishes unless $0 \leq d \leq d^{\prime}$. If $0 \leq d \leq d^{\prime}$ and the rank of $E$ is $2 n$ then (5) identifies canonically with

$$
\epsilon_{!}\left(i_{X}\right)_{!}\left(\left(\wedge^{2} E\right)^{(d)} \otimes E^{\left(d^{\prime}-d\right)}\right)\left[d^{\prime}\right]
$$

We actually prove a more precise claim for any local system $E$ (cf. Proposition 2.3.5 and Remark 2.3.6).

\subsection{Global linear periods}

2.2.1

Let $E$ be an irreducible rank $2 n$ local system on $X$, recall the perverse sheaf $\mathrm{Aut}_{E}$ on Bun $_{2 n}$ from Section 2.1. Consider the diagram

$$
\operatorname{Bun}_{2 n} \stackrel{v_{n}}{\leftarrow} \operatorname{Bun}_{n} \times \operatorname{Bun}_{n} \stackrel{\operatorname{det} \times \operatorname{det}}{\rightarrow} \operatorname{Pic} X \times \operatorname{Pic} X,
$$

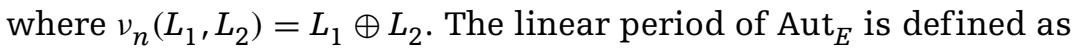

$$
\operatorname{Per}_{E}=(\operatorname{det} \times \operatorname{det}) ! v_{n}^{*} \operatorname{Aut}_{E}\left[\operatorname{dim} \cdot \operatorname{rel}\left(v_{n}\right)\right]
$$

For $d, d^{\prime} \in \mathbb{Z}$ write $\operatorname{Per}_{E}^{d, d^{\prime}}$ for its restriction to the component $(\operatorname{Pic} X \times \operatorname{Pic} X)^{d, d^{\prime}}$.

For a local system $\mathcal{A}$ of rank one on $X$, write $A \mathcal{A}$ for the corresponding automorphic local system on $\operatorname{Pic} X$. This is a character local system such that for any $d \geq 0$ the restriction of $A \mathcal{A}$ under $X^{(d)} \rightarrow \operatorname{Pic} X, D \mapsto \mathcal{O}(D)$ identifies canonically with $\mathcal{A}^{(d)}$.

Let Bun $_{1}$ act on $\operatorname{Pic} X \times \operatorname{Pic} X$ by $\bar{a}: \operatorname{Bun}_{1} \times \operatorname{Pic} X \times \operatorname{Pic} X \rightarrow \operatorname{Pic} X \times \operatorname{Pic} X$ sending $\left(\mathcal{A}, L_{1}, L_{2}\right)$ to $\left(L_{1} \otimes \mathcal{A}^{n}, L_{2} \otimes \mathcal{A}^{n}\right)$. Then, $\operatorname{Per}_{E}$ satisfies the following equivariance property for this action

$$
\bar{a}^{*} \operatorname{Per}_{E} \underset{\rightarrow}{\rightarrow} A(\operatorname{det} E) \otimes \operatorname{Per}_{E}
$$

Note that $\operatorname{Per}_{E}$ is also $S_{2}$-equivariant, where $S_{2}$ permutes the two copies of Pic $X$.

The following is derived from Theorem 2.1.2. 
Theorem 2.2.2. $\quad$ (i) If $d, d^{\prime} \in \mathbb{Z}$ then $\operatorname{Per}_{E}^{d, d^{\prime}}$ vanishes unless $4 n(1-g) \leq d-d^{\prime} \leq 0$. (ii) There is $N \in \mathbb{Z}$ such that for any $d, d^{\prime} \in \mathbb{Z}, \operatorname{Per}_{E}^{d, d^{\prime}}$ is placed in usual cohomological degrees $\leq N$. If $0 \leq d \leq d^{\prime}$ and $d \in \mathbb{Z}$ is large enough then one has

$$
\tau_{\geq N^{\prime}} P e r_{E}^{d, d^{\prime}} \underset{\rightarrow}{\sim} \tau_{\geq N^{\prime}}\left(\epsilon_{!}\left(i_{X}\right)_{!}\left(\left(\wedge^{2} E\right)^{(d)} \otimes E^{\left(d^{\prime}-d\right)}\right)\left[d^{\prime}+d+(g-1)\left(n-2 n^{2}\right)\right]\right)
$$

over $(\operatorname{Pic} X \times \operatorname{Pic} X)^{d, d^{\prime}}$ with

$$
N^{\prime}=N-2 d+(2 g-2)\left(2 n^{2}-n\right)+2
$$

Taking into account the equivariance property (8), this gives a description of all the left truncations of $\operatorname{Per}_{E}^{d, d^{\prime}}$ for all $d, d^{\prime} \in \mathbb{Z}$.

Remark 2.2.3. We did not look for the constant $N$ in general. One checks that for any $d \in \mathbb{Z}$ the complex $\operatorname{Per}_{E}^{d, d}$ is placed in usual degrees $\leq 2+(g-1)\left(2 n^{2}-n-2\right)$.

\subsection{Plan of the proof of Theorem 2.1.2}

\subsection{1}

For $d \geq 0$, consider the stack $\mathcal{Y}_{m, d}$ classifying $L \in$ Bun $_{m}$ together with sections

$$
t_{i}: \Omega^{(m-1)+(m-2)+\ldots+(m-i)} \hookrightarrow \wedge^{i} L
$$

for $i=1, \ldots, m$ satisfying the Plücker relations, $D \in X^{(d)}$ such that

$$
t_{m}: \Omega^{(m-1)+(m-2)+\ldots+1}(D) \stackrel{\sim}{\rightarrow} \operatorname{det} L
$$

is an isomorphism ([11], Section 4.1). For a local system $E$ on $X$, we have a perverse sheaf $\mathcal{P}_{m, E}^{d}$ on $\mathcal{Y}_{m, d}$ introduced in ([11], 4.2, Def. 2), see also Section 3.1.1. Let $q_{\mathcal{Y}}: \mathcal{Y}_{m, d} \rightarrow \mathcal{M}_{m, d}$ be the map sending the above point to $\left(L, t_{1}\right)$. By definition, $\mathcal{K}_{m, E}^{d} \widetilde{\rightarrow}\left(q_{\mathcal{Y}}\right)_{!}\left(\mathcal{P}_{m, E}^{d}\right)$ for $d \geq 0$.

\subsection{2}

Let $\widetilde{\mathcal{Y}}_{n}$ be the stack classifying $L \in \operatorname{Bun}_{n}$ with regular sections

$$
s_{i}: \Omega^{(2 n-1)+(2 n-3)+\ldots+(2 n-2 i+1)} \hookrightarrow \wedge^{i} L
$$


for $1 \leq i \leq n$ over $X$ satisfying the Plücker relations. For $d \geq 0$ the connected component $\widetilde{\mathcal{Y}}_{n, d}$ of $\widetilde{\mathcal{Y}}_{n}$ is defined by the property that there is $D \in X^{(d)}$ such that

$$
s_{n}: \Omega^{(2 n-1)+\ldots+1}(D) \stackrel{\operatorname{det} L}{\Im}
$$

is an isomorphism. Let similarly $\widetilde{\mathcal{Y}}_{n}^{\prime}$ be the stack classifying $L^{\prime} \in \operatorname{Bun}_{n}$ with regular sections

$$
s_{i}^{\prime}: \Omega^{(2 n-2)+(2 n-4)+\ldots+(2 n-2 i)} \hookrightarrow \wedge^{i} L^{\prime}
$$

for $1 \leq i \leq n$ satisfying the Plücker relations. For $d^{\prime} \geq 0$, the connected component $\tilde{\mathcal{Y}}_{n, d^{\prime}}^{\prime}$ of $\widetilde{\mathcal{Y}}_{n}^{\prime}$ is defined by the property that there is $D^{\prime} \in X^{\left(d^{\prime}\right)}$ such that

$$
s_{n}^{\prime}: \Omega^{(2 n-2)+\ldots+2}\left(D^{\prime}\right) \stackrel{\sim}{\rightarrow} \operatorname{det} L^{\prime}
$$

is an isomorphism. Let

$$
\bar{\pi}: \widetilde{\mathcal{Y}}_{n, d} \times \widetilde{\mathcal{Y}}_{n, d^{\prime}}^{\prime} \rightarrow X^{(d)} \times X^{\left(d^{\prime}\right)}
$$

be the map sending the above point to $\left(D, D^{\prime}\right)$.

Let $\iota: \widetilde{\mathcal{Y}}_{n, d} \times \widetilde{\mathcal{Y}}_{n, d^{\prime}}^{\prime} \rightarrow \mathcal{Y}_{2 n}$ be the map sending the above point to $\left(M,\left(t_{k}\right)\right)$, where $M=L \oplus L^{\prime}$ and $t_{k}$ are defined as follows. For $1 \leq 2 k \leq 2 n$, we get maps

$$
s_{k} \otimes s_{k}^{\prime}: \Omega^{(2 n-1)+\ldots+(2 n-2 k)} \hookrightarrow\left(\wedge^{k} L\right) \otimes\left(\wedge^{k} L^{\prime}\right) \subset \wedge^{2 k} M
$$

For $1<2 k+1 \leq 2 n$, we get maps

$$
s_{k+1} \otimes s_{k}^{\prime}: \Omega^{(2 n-1)+\ldots+(2 n-2 k-1)} \hookrightarrow\left(\wedge^{k+1} L\right) \otimes\left(\wedge^{k} L^{\prime}\right) \subset \wedge^{2 k+1} M
$$

Set

$$
\begin{aligned}
& t_{2 k}=(-1)^{1+\ldots+(k-1)} s_{k} \otimes s_{k^{\prime}}^{\prime} \quad \text { for } 1 \leq 2 k \leq 2 n \\
& t_{2 k+1}=(-1)^{1+\ldots+k} s_{k+1} \otimes s_{k^{\prime}}^{\prime} \quad \text { for } 1<2 k+1 \leq 2 n \\
& t_{1}=s_{1}
\end{aligned}
$$

These $\left(t_{k}\right)$ automatically satisfy the Plücker relations, so that $\iota$ takes values in $\mathcal{Y}_{2 n}$. 


\section{3 .3}

We get the commutative diagram

$$
\begin{array}{ccccc}
\widetilde{\mathcal{Y}}_{n, d} \times \tilde{\mathcal{Y}}_{n, d^{\prime}}^{\prime} & \stackrel{\bar{\pi}}{\rightarrow} & X^{(d)} \times X^{\left(d^{\prime}\right)} & & \\
& \downarrow q_{\mathcal{Z}} & & & \searrow \epsilon \\
\mathcal{Y}_{2 n} \times \mathcal{M}_{2 n} \mathcal{Z}_{n} & \stackrel{\operatorname{pr}_{2}}{\rightarrow} & \mathcal{Z}_{n} & \stackrel{\pi}{\rightarrow} & \operatorname{Pic} X \times \operatorname{Pic} X \\
\downarrow p_{1} & & \downarrow v_{\mathcal{Z}} & & \\
\mathcal{Y}_{2 n} & \rightarrow & \mathcal{M}_{2 n} & &
\end{array}
$$

Here $q_{\mathcal{Y Z}}$ is the map whose projection to $\mathcal{Y}_{2 n}$ is $\iota$, and its projection to $\mathcal{Z}_{n}$ sends the corresponding point to $\left(L, L^{\prime}, t_{1}\right)$.

For any local system $E$ on $X$, we will define a canonical constructible subsheaf

$$
{ }^{\leq n}\left(\left(\wedge^{2} E\right)^{(d)} \otimes E^{\left(d^{\prime}-d\right)}\right) \subset\left(\wedge^{2} E\right)^{(d)} \otimes E^{\left(d^{\prime}-d\right)}
$$

on $X^{(d)} \times X^{\left(d^{\prime}-d\right)}$ in Definition 4.3.11. We refer the reader to loc.cit. for the precise construction. As $n$ varies, they form an increasing filtration on $\left(\wedge^{2} E\right)^{(d)} \otimes E^{\left(d^{\prime}-d\right)}$.

Theorem 2.1.2 is immediately reduced to Propositions 2.3.4 and 2.3.5.

Proposition 2.3.4. Let $d+d^{\prime} \geq 0$. For any local system $E$ on $X$, the natural map

$$
\pi_{!} \operatorname{pr}_{2 !}\left(\mathcal{P}_{2 n, E}^{d+d^{\prime}} \otimes \overline{\mathbb{Q}}_{\ell}\right) \rightarrow \pi_{!} \operatorname{pr}_{2 !}\left(q_{\mathcal{Y Z}}\right)_{*} q_{\mathcal{Y} \mathcal{Z}}^{*}\left(\mathcal{P}_{2 n, E}^{d+d^{\prime}} \otimes \overline{\mathbb{Q}}_{\ell}\right)
$$

is an isomorphism. In particular, it vanishes unless $d, d^{\prime} \geq 0$. Besides, $q_{\mathcal{Y Z}}$ is a closed immersion.

Proposition 2.3.5. Let $d, d^{\prime} \geq 0$. For any local system $E$ on $X$, there is a canonical isomorphism

$$
\bar{\pi}_{!} q_{\mathcal{Y} \mathcal{Z}}^{*}\left(\mathcal{P}_{2 n, E}^{d+d^{\prime}} \otimes \overline{\mathbb{Q}}_{\ell}\right)\left[\operatorname{dim} \cdot \operatorname{rel}\left(v_{\mathcal{Z}}\right)\right] \underset{\rightarrow}{\rightarrow}\left(i_{X}\right)_{!}\left({ }^{\leq n}\left(\left(\wedge^{2} E\right)^{(d)} \otimes E^{\left(d^{\prime}-d\right)}\right)\right)\left[d^{\prime}\right]
$$

If $E$ is of rank $2 n$ then (12) is an equality. In particular, (13) vanishes unless $d \leq d^{\prime}$.

The proof of Proposition 2.3.4 (resp., 2.3.5) is the purpose of Section 3 (resp., 4).

Remark 2.3.6. The situation here is similar to The Main local theorem of [11], where in calculating some period for a pair of local systems $E, E^{\prime}$ on $X$ we obtained as an answer the subsheaf $\leq^{n}\left(E \otimes E^{\prime}\right)^{(d)} \subset\left(E \otimes E^{\prime}\right)^{(d)}$. 


\subsection{Other results}

2.4 .1

For a finite-dimensional $\overline{\mathbb{Q}}_{\ell}$-vector space $V$ and $\lambda \in \Lambda_{2 n}^{\succ+}$ consider the polynomial functor $V^{\lambda}$ of $V$ defined in ([11], Section 0.1.4). For $\lambda \in \Lambda_{2 n}$, let $\lambda^{\text {odd }}=\left(\lambda_{1}, \lambda_{3}, \ldots, \lambda_{2 n-1}\right)$, $\lambda^{\text {even }}=$ $\left(\lambda_{2}, \lambda_{4}, \ldots, \lambda_{2 n}\right)$.

Lemma 2.4.2. Assume $\operatorname{dim} V=2 n$. Then for $0 \leq d \leq d^{\prime}$ one has

$$
\operatorname{Sym}^{d}\left(\wedge^{2} V\right) \otimes \operatorname{Sym}^{d^{\prime}-d} V \underset{\rightarrow}{\rightarrow} \oplus_{\lambda} V^{\lambda}
$$

as representations of GL(V), the sum being taken over $\lambda \in \Lambda_{2 n, d+d^{\prime}}^{\succ+}$ such that $\lambda^{\text {odd }} \in \Lambda_{n, d^{\prime}}^{\succ+}$ and $\lambda^{\text {even }} \in \Lambda_{n, d}^{\succ+}$. In particular, it is multiplicity free.

Proof. Set temporarily

$$
\mathcal{J}_{d^{\prime}, d}=\left\{\lambda \in \Lambda_{2 n, d+d^{\prime}}^{\succ+} \mid \lambda^{\text {odd }} \in \Lambda_{n, d^{\prime}}^{\succ+} \lambda^{\text {even }} \in \Lambda_{n, d}^{\succ+}\right\}
$$

Consider the map $f: \mathcal{J}_{d^{\prime}, d} \rightarrow \mathcal{J}_{d, d}$ sending $\lambda$ to the unique $\mu \in \mathcal{J}_{d, d}$ such that $\mu^{\text {odd }}=$ $\mu^{\text {even }}=\lambda^{\text {even }}$.

1) The case $d=d^{\prime}$ is ([8], Proposition 1). Moreover, in this case for $\lambda \in \mathcal{J}_{d, d}$, we get $\lambda^{\text {odd }}=\lambda^{\text {even }}$. Indeed, for such $\lambda$ one has $\lambda_{2 i-1} \geq \lambda_{2 i}$ for $1 \leq i \leq n$, and

$$
\sum_{i=1}^{n} \lambda_{2 i-1}=\sum_{i=1}^{n} \lambda_{2 n}=d
$$

so $\lambda_{2 i-1}=\lambda_{2 i}$ for all $i$.

2) The general case reduces to the case $d=d^{\prime}$ using Pieri's rule for representations of $\mathrm{GL}_{2 n}$ found in ([16], Proposition 2.1). Namely, if $\lambda \in \Lambda_{2 n, 2 d}^{\succ+}$ with $\lambda^{\text {odd }}=\lambda^{\text {even }}$ then Pieri's rule gives $V^{\lambda} \otimes \operatorname{Sym}^{d^{\prime}-d} V \widetilde{\rightarrow} \oplus_{\mu} V^{\mu}$, the sum being over $\mu \in \mathcal{J}_{d^{\prime}, d}$ with $f(\mu)=\lambda$. So,

$$
\left.\operatorname{Sym}^{d}\left(\wedge^{2} V\right) \otimes \operatorname{Sym}^{d^{\prime}-d} V \widetilde{\rightarrow} \underset{\lambda \in \mathcal{J}_{d, d}}{\oplus} V^{\lambda} \otimes \operatorname{Sym}^{d^{\prime}-d} V \widetilde{\rightarrow} \underset{\lambda \in \mathcal{J}_{d, d}}{\oplus} \underset{\substack{\mu \in \mathcal{J}_{d^{\prime}, d} \\ f(\mu)=\lambda}}{\oplus} V_{\mu}\right) \underset{\rightarrow}{\rightarrow} \underset{\mu \in \mathcal{J}_{d^{\prime}, d}}{\oplus} V_{\mu}
$$




\section{4 .3}

Let $0 \leq d \leq d^{\prime}$. For $\lambda \in \Lambda_{2 n, d+d^{\prime}}^{\succ-}$ with $\lambda^{\text {odd }} \in \Lambda_{n, d^{\prime}}^{\succ-} \lambda^{\text {even }} \in \Lambda_{n, d^{\prime}}^{\succ-}$ let

$$
\operatorname{sum}^{\lambda}: X_{-}^{\lambda} \rightarrow X^{(d)} \times X^{\left(d^{\prime}\right)}
$$

be the map sending $\left(D_{1}, \ldots, D_{2 n}\right)$ to $\left(D, D^{\prime}\right)$ with $D=D_{1}+D_{3}+\ldots+D_{2 n-1}$ and $D^{\prime}=$ $D_{2}+D_{4}+\ldots+D_{2 n}$.

We think of the following informally as a version of Lemma 2.4.2, where a point is replaced by the curve $X$. The proof of Proposition 2.4.4 is given in Section 4.3.13.

Proposition 2.4.4. For any local system $E$ on $X$, the sheaf $\leq n\left(\left(\wedge^{2} E\right)^{(d)} \otimes E^{\left(d^{\prime}-d\right)}\right)$ admits a filtration with the associated graded

$$
\underset{\lambda}{\oplus} \operatorname{sum}_{!}^{\lambda} E_{-,}^{\lambda}
$$

the sum being taken over $\lambda \in \Lambda_{2 n, d+d^{\prime}}^{\succ-}$ such that $\lambda^{\text {odd }} \in \Lambda_{n, d}^{\succ-}$ and $\lambda^{\text {even }} \in \Lambda_{n, d^{\prime}}^{\succ-}$. Moreover, if $\operatorname{rk}(E)=2 n$, then (12) is an equality.

\section{4 .5}

Among other results, we underline Theorem 4.3.9, which is of independent interest. We refer the reader to Section 4.2 for its formulation. One could think of it as an analog of ([11], Theorem C) in our setting.

Remark 2.4.6. The following phenomenon appears in Theorem 4.3.9, it has also appeared in the local Rankin-Selberg method for $\mathrm{GL}_{n}$ in [11]. Let $f: Y \rightarrow Z$ be a morphism of stacks, $Z$ a scheme of finite type, $\mathcal{E}$ the set of irreducible components of $Z$. Assume the normalization of $Z$ is of the form norm $: \sqcup_{i \in \mathcal{E}} Z_{i} \rightarrow Z$, where $Z_{i}$ is smooth irreducible, the image $\operatorname{norm}\left(Z_{i}\right)$ is the $i$ th irreducible component of $Z$. Assume all the fibres of $f$ are of dimension $\leq d$ for some $d \in \mathbb{Z}$. To calculate $\mathrm{R}^{2 d} f_{!} \overline{\mathbb{Q}}_{\ell}$, we find a stratification of $Y$ by locally closed substacks $Y_{i}, i \in \mathcal{E}$ with the property that the map $Y_{i} \rightarrow Z$ factors naturally as $Y_{i} \stackrel{f_{i}}{\rightarrow} Z_{i} \stackrel{\text { norm }}{\rightarrow} Z$ and establish isomorphisms $\mathrm{R}^{2 d}\left(f_{i}\right)_{!} \overline{\mathbb{Q}}_{\ell} \underset{\rightarrow}{\rightarrow} \overline{\mathbb{Q}}_{\ell}$ for $i \in \mathcal{E}$. Then

$$
\mathrm{R}^{2 d} f_{!} \overline{\mathbb{Q}}_{\ell} \widetilde{\sim} \text { norm }_{!} \overline{\mathbb{Q}}_{\ell}
$$

Indeed, this direct image has a filtration with the associated graded $\oplus_{i \in \mathcal{E}}(\text { norm })_{i} \overline{\mathbb{Q}}_{\ell}$, and any such filtration splits canonically. 


\subsection{Relation with the classical theory}

2.5 .1

For $d, d^{\prime} \in \mathbb{Z}$ let $\mathcal{Z}_{n}^{d, d^{\prime}} \subset \mathcal{Z}_{n}$ be the component given by $\left(\operatorname{det} L, \operatorname{det} L^{\prime}\right) \in(\operatorname{Pic} X \times \operatorname{Pic} X)^{d, d^{\prime}}$ for $\left(L, L^{\prime}, s: \Omega^{2 n-1} \hookrightarrow L\right) \in \mathcal{Z}_{n}$.

From Theorem 2.1.2 one derives the following.

Corollary 2.5.2. Let $0 \leq d \leq d^{\prime}$ and $E$ be a local system of rank $2 n$ on $X$. Let $V_{1}, V_{2}$ be local systems of rank one on $X$. One has

$$
\begin{aligned}
& \mathrm{R} \Gamma_{c}\left(\mathcal{Z}_{n}^{d, d^{\prime}}, v_{\mathcal{Z}}^{*} \mathcal{K}_{2 n, E}^{d+d^{\prime}} \otimes \pi^{*}\left(A V_{1} \otimes A V_{2}\right)\right)[n(g-1)] \underset{\Im}{ } \\
& V_{0} \otimes \mathrm{R} \Gamma\left(X^{(d)} \times X^{\left(d^{\prime}-d\right)},\left(V_{1} \otimes V_{2} \otimes \wedge^{2} E\right)^{(d)} \otimes\left(V_{2} \otimes E\right)^{\left(d^{\prime}-d\right)}\right)\left[2 d^{\prime}\right]
\end{aligned}
$$

Here

$$
V_{0}=\left(A V_{1}\right)_{\Omega^{(2 n-1)+(2 n-3)+\ldots+1}} \otimes\left(A V_{2}\right)_{\Omega^{(2 n-2)+(2 n-4)+\ldots+2}}
$$

If $d, d^{\prime} \in \mathbb{Z}$ with $d+d^{\prime} \geq 0$ then the left hand side of (14) vanishes unless $0 \leq d \leq d^{\prime}$

2.5 .3

For this subsection, assume the base field is $\mathbb{F}_{q}$. For a local system $E$ on $X$, the L-function of $E$ is defined as the formal series in $\overline{\mathbb{Q}}_{\ell}[[t]]$

$$
L(E, t)=\sum_{d \geq 0} \sum_{D \in X^{(d)}\left(\mathbb{F}_{q}\right)} \operatorname{tr}\left(\mathrm{Fr}_{D^{\prime}} E^{(d)}\right) t^{d}
$$

According to the Grothendieck's trace formula,

$$
L(E, t)=\prod_{r=0}^{2} \operatorname{det}\left(1-t \mathrm{Fr}, \mathrm{H}^{r}\left(X \otimes_{\mathbb{F}_{q}} k, E\right)\right)^{(-1)^{r+1}}
$$

Here Fr is the geometric Frobenius endomorphism. For an irreducible local system $E$ of rank $2 n$ on $X$ let

$$
\varphi_{E}: \operatorname{Bun}_{2 n}\left(\mathbb{F}_{q}\right) \rightarrow \overline{\mathbb{Q}}_{\ell}
$$

be the function trace of Frobenius of Aut $_{E}$. Let $\varphi_{V_{i}}:(\operatorname{Pic} X)\left(\mathbb{F}_{q}\right) \rightarrow \overline{\mathbb{Q}}_{\ell}$ be the function trace of Frobenius of $A V_{i}$ for rank one local systems $V_{i}$. Then, Corollary 2.5.2 yields the 
equality in $\overline{\mathbb{Q}}_{\ell}[[t]]$

$$
\begin{aligned}
\sum_{d \geq 0} \sum_{\left(s, L, L^{\prime}\right) \in \mathcal{Z}_{n}^{d, d}\left(\mathbb{F}_{q}\right)} \frac{1}{\sharp \operatorname{Aut}\left(\Omega^{2 n-1} \hookrightarrow L\right) \sharp \operatorname{Aut}\left(L^{\prime}\right)} \varphi_{E}\left(L \oplus L^{\prime}\right) \varphi_{V_{1}}(\operatorname{det} L) \varphi_{V_{2}}\left(\operatorname{det} L^{\prime}\right) t^{d}= \\
q^{\left(n-4 n^{2}\right)(g-1) / 2} \operatorname{tr}\left(\operatorname{Fr}, V_{0}\right) L\left(V_{1} \otimes V_{2} \otimes \wedge^{2} E, t\right)
\end{aligned}
$$

Here $\sharp A$ denotes the number of element of the set $A$.

If $d$ is large enough and $\varphi_{E}\left(L \oplus L^{\prime}\right) \neq 0$ then $\operatorname{Ext}^{1}\left(\Omega^{2 n-1}, L\right)=0$ and one has $\operatorname{dim} \operatorname{Hom}\left(\Omega^{2 n-1}, L\right)=d+(g-1)\left(n-2 n^{2}\right)$. One may derive some results of [4] about linear periods by passing to the residue at $t=q^{-1}$ in the above equality.

\subsection{Application: automorphic sheaves for $\mathrm{GSp}_{4}$}

\subsection{1}

Use the following notations from ([14], Section 3.3.1). For a reductive group $G$ over $k, \breve{G}$ denotes its Langlands dual over $\overline{\mathbb{Q}}_{\ell}$. Let $\mathbb{G}=\mathrm{GSp}_{4}, E_{\breve{G}}$ be a $\breve{G}$-local system on $X$ viewed as a pair $(E, \chi)$, where $E$ (resp., $\chi$ ) is of rank 4 (resp., 1 ) local system on $X$ equipped with a symplectic form $\wedge^{2} E \rightarrow \chi$.

Let $\mathbb{H}=\mathrm{GO}_{6}^{0}$ and $\kappa: \check{\breve{G}} \hookrightarrow \check{\mathbb{H}}$ be as in loc.cit. So, $\check{\mathbb{H}} \widetilde{\rightarrow}\left\{(c, b) \in \mathbb{G}_{m} \times \mathrm{GL}_{4} \mid\right.$ $\left.\operatorname{det} b=c^{2}\right\}$, and $\kappa$ is the natural inclusion. Let $E_{\breve{H}}$ be the $\check{\mathbb{H}}$-local system on $X$ obtained via extension of scalars via $\kappa$. Thus, $E_{\breve{H}}$ is given by the pair $(E, \chi)$ with the induced isomorphism $\operatorname{det} E \widetilde{\sim} \chi^{2}$ on $X$. Let $F_{\mathbb{G}}: \mathrm{D}^{-}\left(\mathrm{Bun}_{\mathbb{H}}\right) \rightarrow \mathrm{D}^{\prec}\left(\mathrm{Bun}_{\mathbb{G}}\right)$ be the theta-lifting functor from ([14], Section 2.2.6).

Assume $E$ irreducible on $X$. Under these assumptions, we constructed the $E_{\breve{G}^{-}}$ Hecke eigensheaf denoted $F_{\mathbb{G}}\left(K_{E^{*}, \chi^{*}, \mathbb{H}}\right) \in \mathrm{D}^{\prec}\left(\operatorname{Bun}_{\mathbb{G}}\right)$ in ([14], Theorem 3.3.4).

Theorem 2.6.2. Under the assumptions of Section 2.6 .1 , the complex $F_{\mathbb{G}}\left(K_{E^{*}, \chi^{*}, \mathbb{H}}\right)$ is nonzero.

\section{Passing to a closed substack}

In this section, we prove Proposition 2.3.4. In Section 3.1.1, we describe some general properties of the perverse sheaf $\mathcal{P}_{n, E}^{d}$, namely its $*$-restriction to the natural stratification, and related results. After this preparation, the proof of Proposition 2.3.4 given in Section 3.3.7 is a combination of Propositions 3.2.3, Lemma 3.3.6, and Corollary 3.3.4. 
Namely, we will decompose $q_{\mathcal{Y Z}}$ into closed immersions

$$
\tilde{\mathcal{Y}}_{n, d} \times \widetilde{\mathcal{Y}}_{n, d^{\prime}}^{\prime} \stackrel{i_{\mathcal{Y}}}{\rightarrow} 2 n \mathcal{Z}^{d, d^{\prime}} \stackrel{2 n_{\kappa}}{\rightarrow} \mathcal{Y}_{2 n} \times \mathcal{M}_{2 n} \mathcal{Z}_{n}
$$

and successively replace the integration over $\mathcal{Y}_{2 n} \times \mathcal{M}_{2 n} \mathcal{Z}_{n}$ by the integration over a smaller substack in the above diagram.

\subsection{Generalities about $\mathcal{P}_{n, E}^{d}$}

3.1 .1

For $r \geq 0$ let $\mathcal{Q}_{n, r}$ be the stack classifying collections

$$
\left(0=L_{0} \subset \ldots \subset L_{n} \subset L_{,}\left(s_{i}\right)\right),
$$

where $L_{n} \subset L$ is a modification of rank $n$ vector bundles on $X$ with $\operatorname{deg}\left(L / L_{n}\right)=r,\left(L_{i}\right)$ is a complete flag of subbundles on $L_{n}$, and $s_{i}: \Omega^{n-i} \underset{\Im}{\rightarrow} L_{i} / L_{i-1}$ is an isomorphism for $i=1, \ldots, n$. We have the diagram $\mathbb{A}^{1} \stackrel{\mu}{\leftarrow} \mathcal{Q}_{n, r} \stackrel{\beta}{\rightarrow} \leq n \operatorname{Sh}_{0}^{r}$ as in ([11], Section 2.1), here $\beta$ sends the above point to $L / L_{n}$. As in loc.cit., for a local system $E$ on $X$, one defines the perverse sheaf

$$
\mathcal{F}_{n, E}^{r}=\beta^{*} \mathcal{L}_{E}^{r} \otimes \mu^{*} \mathcal{L}_{\psi}[b(n, r)]
$$

with

$$
b(n, r)=n r+(1-g) \sum_{i=1}^{n-1} i^{2}=\operatorname{dim}\left(\mathcal{Q}_{n, r}\right)
$$

Let $\varphi: \mathcal{Q}_{n, r} \rightarrow \mathcal{Y}_{n, r}$ be the map sending the above point to $\left(L,\left(t_{i}\right)\right)$, where

$$
t_{i}: \Omega^{(n-1)+\ldots+(n-i)} \underset{\rightarrow}{\sim} \wedge^{i} L_{i} \hookrightarrow \wedge^{i} L
$$

are the induced maps for $i=1, \ldots, n$. By definition, $\mathcal{P}_{n, E}^{r} \underset{\rightarrow}{\rightarrow} \varphi_{!}\left(\mathcal{F}_{n, E}^{r}\right)$. Write $\widetilde{\mathcal{P}}_{n, E}^{r}$ for the complex on $\mathcal{Y}_{n, r}$ obtained from $\mathcal{P}_{n, E}^{r}$ by replacing in its definition $\mathcal{L}_{E}^{r}$ by $\mathcal{S p r}_{E}^{r}$ (as in [11], p. 489 after Lemma 12).

\section{1 .2}

As in ([11], Section 4.1), we stratify $\mathcal{Y}_{n, d}$ by locally closed substacks $S(\mathcal{Y})_{\text {pos }}^{\lambda}$ indexed by $\lambda \in \Lambda_{n, d}^{>\text {pos }}$. The stratum $S(\mathcal{Y})_{\text {pos }}^{\lambda}$ is given by requiring that the degree of zeros of 
$t_{i}: \Omega^{(n-1)+\ldots+(n-i)} \hookrightarrow \wedge^{i} L$ equals $\lambda_{1}+\ldots+\lambda_{i}$ for $i=1, \ldots, n$. So, $S(\mathcal{Y})_{p o s}^{\lambda}$ classifies collections: $\left(D_{i}\right) \in X_{p o s}^{\lambda}$, a complete flag of vector bundles $\left(0=L_{0} \subset L_{1} \subset \ldots \subset L_{n}=L\right)$ on $L \in \operatorname{Bun}_{n}$ with trivializations

$$
\Omega^{(n-1)+\ldots+(n-i)}\left(D_{1}+\ldots+D_{i}\right) \widetilde{\neg} \wedge^{i} L_{i}
$$

Let $S(\mathcal{Y})_{-}^{\lambda}=S(\mathcal{Y})_{\text {pos }}^{\lambda} \times_{X_{\text {pos }}^{\lambda}} X_{-}^{\lambda}$. We have a map $\mu_{\lambda}: S(\mathcal{Y})_{-}^{\lambda} \rightarrow \mathbb{A}^{1}$ sending the above point to the sum in $k \widetilde{\operatorname{Ext}^{1}}\left(\Omega^{n-i-1}\left(D_{i}\right), \Omega^{n-i}\left(D_{i}\right)\right)$ over $1 \leq i<n$ of the pullbacks of

$$
0 \rightarrow L_{i} / L_{i-1} \rightarrow L_{i+1} / L_{i-1} \rightarrow L_{i+1} / L_{i} \rightarrow 0
$$

under

$$
\Omega^{n-i-1}\left(D_{i}\right) \hookrightarrow \Omega^{n-i-1}\left(D_{i+1}\right) \Im L_{i+1} / L_{i}
$$

Let $q_{-}^{\lambda}: S(\mathcal{Y})_{-}^{\lambda} \rightarrow X_{-}^{\lambda}$ be the projection sending the above point to $\left(D_{i}\right)$. For $\lambda \in \mathbb{Z}^{n}$ set

$$
a_{n}(\lambda)=\langle\lambda,(n-1, n-2, \ldots, 0)\rangle
$$

as in [11]. For $\lambda \in \Lambda_{n, d}^{\succ-}$ recall the definition of the sheaf $E_{-}^{\lambda}$ on $X_{-}^{\lambda}$ from ([11], Definition 1). Let $f_{\lambda}: X_{-}^{\lambda} \rightarrow{ }^{n} \operatorname{Sh}_{0}^{d}$ be the map sending $\left(D_{1}, \ldots, D_{n}\right)$ to

$$
\Omega^{n-1}\left(D_{1}\right) / \Omega^{n-1} \oplus \Omega^{n-2}\left(D_{2}\right) / \Omega^{n-2} \oplus \ldots \oplus \mathcal{O}\left(D_{n}\right) / \mathcal{O}
$$

For any smooth $\overline{\mathbb{Q}}_{\ell}$-sheaf $E$ on $X$ set

$$
E_{-}^{\lambda}=\mathcal{H}^{2 a_{n}(\lambda)}\left(f_{\lambda}^{*} \mathcal{L}_{E}^{d}\right)
$$

where $\mathcal{H}^{i}$ denotes the $i$-cohomology sheaf for the usual t-structure.

We will use the following.

Proposition 3.1.3. [11], Proposition 2). Let $E$ be a smooth $\overline{\mathbb{Q}}_{\ell}$-sheaf on $X$ of rank $m$, $\lambda \in \Lambda_{n, d}^{\succ \text { pos }}$. The $*$-restriction of $\mathcal{P}_{n, E}^{d}$ to $S(\mathcal{Y})_{\text {pos }}^{\lambda}$ vanishes unless $\lambda_{1}=\ldots=\lambda_{n-m}=0$ and $\lambda \in \Lambda_{n, d}^{\succ-}$. In the latter case, it is the extension by zero under $S(\mathcal{Y})_{-}^{\lambda} \hookrightarrow S(\mathcal{Y})_{\text {pos }}^{\lambda}$ of

$$
\left(q_{-}^{\lambda}\right)^{*} E_{-}^{\lambda} \otimes \mu_{\lambda}^{*} \mathcal{L}_{\psi}\left[b(n, d)-2 a_{n}(\lambda)\right]
$$


3.1 .4

For $1 \leq j \leq m$ let ${ }_{j} \mathcal{Y}_{m}$ be the stack classifying $L \in$ Bun $_{m}$ together with sections (9) for $1 \leq i \leq j$ satisfying the Plücker relations. For $j<m$ let $\delta_{j}: \mathcal{Y}_{m} \rightarrow{ }_{j+1} \mathcal{Y}_{m}$ be the projection forgetting $t_{j+2}, \ldots, t_{m}$.

Given $\lambda \in \Lambda_{j}^{\succ \text { pos }}$ consider the locally closed substack ${ }_{j+1} \mathcal{Y}_{m}^{\lambda} \hookrightarrow{ }_{j+1} \mathcal{Y}_{m}$ given by the property that there is $\left(D_{1}, \ldots, D_{j}\right) \in X_{\text {pos }}^{\lambda}$ such that

$$
t_{i}: \Omega^{(m-1)+\ldots+(m-i)}\left(D_{1}+\ldots+D_{i}\right) \hookrightarrow \wedge^{i} L
$$

is a subbundle for $1 \leq i \leq j$. A point of ${ }_{j+1} \mathcal{Y}_{m}^{\lambda}$ gives a flag $\left(L_{1} \subset \ldots \subset L_{j} \subset L\right)$ of vector bundles on $X$ with trivializations for $1 \leq i \leq j$

$$
L_{i} / L_{i-1} \stackrel{\sim}{\rightarrow} \Omega^{m-i}\left(D_{i}\right)
$$

and a section $t_{j+1}: \Omega^{(m-1)+\ldots+(m-j-1)} \hookrightarrow \wedge^{j} L_{j} \otimes L / L_{j}$.

Let ${ }_{j+1} \mathcal{Y}_{m,-}^{\lambda}$ be the closed substack of ${ }_{j+1} \mathcal{Y}_{m}^{\lambda}$ given by the properties: $0 \leq D_{1} \leq$ $\ldots \leq D_{j}$, and there is a regular section $s: \Omega^{m-j-1}\left(D_{j}\right) \hookrightarrow L / L_{j}$ such that

$$
t_{j+1}=t_{j} \otimes s: \Omega^{(m-1)+\ldots+(m-j-1)} \hookrightarrow \wedge^{j} L_{j} \otimes L / L_{j} \subset \wedge^{j+1} L
$$

We used the fact that $t_{j}: \Omega^{(m-1)+\ldots+(m-j)} \hookrightarrow \wedge^{j} L_{j}$.

Let ${ }_{j} \mathcal{W}^{\lambda}$ be the stack classifying $\left(D_{1}, \ldots, D_{j}\right) \in X_{-}^{\lambda}, \bar{L} \in \operatorname{Bun}_{m-j}$ with a section $s: \Omega^{m-j-1}\left(D_{j}\right) \hookrightarrow \bar{L}$. Let

$$
\tau^{\lambda}:{ }_{j+1} \mathcal{Y}_{m,-}^{\lambda} \rightarrow{ }_{j} \mathcal{W}^{\lambda}
$$

be the map sending the above point to $\left(\bar{L},\left(D_{i}\right), s\right)$ with $\bar{L}=L / L_{j}$. Let ev $:{ }_{j+1} \mathcal{Y}_{m,-}^{\lambda} \rightarrow \mathbb{A}^{1}$ be the map sending a point of ${ }_{j+1} \mathcal{Y}_{m,-}^{\lambda}$ to the sum in $\mathrm{H}^{1}(X, \Omega) \stackrel{\sim}{\rightarrow} \mathbb{A}^{1}$ of the pull-back extensions

$$
0 \rightarrow L_{i} / L_{i-1} \rightarrow L_{i+1} / L_{i} \rightarrow \underset{\uparrow}{\uparrow} \begin{gathered}
L_{i+1} / L_{i} \\
\Omega^{m-i-1}\left(D_{i}\right)
\end{gathered}
$$

for $1 \leq i \leq j-1$ and the pull-back of $0 \rightarrow L_{j} / L_{j-1} \rightarrow L / L_{j-1} \rightarrow L / L_{j} \rightarrow 0$ under $s:$ $\Omega^{m-j-1}\left(D_{j}\right) \hookrightarrow L / L_{j}$ 
Lemma 3.1.5. Let $1 \leq j<m, E$ be any local system on $X$. The $*$-restriction of $\delta_{j !}\left(\mathcal{P}_{m, E}\right)$ to ${ }_{j+1} \mathcal{Y}_{m}^{\lambda}$ is the extension by zero from ${ }_{j+1} \mathcal{Y}_{m,-}^{\lambda}$ of $e v^{*} \mathcal{L}_{\psi} \otimes\left(\tau^{\lambda}\right)^{*} K^{\prime}$ for some complex $K^{\prime} \in \mathrm{D}\left({ }_{j} \mathcal{W}^{\lambda}\right)$.

Proof. This follows from the equivariance properties of Whittaker sheaves ([5], Proposition 4.13) and ([11], Proposition 2). A similar argument is also used in ([6], Sections 4.4 and 4.5).

Namely, denote temporarily by $\mathcal{P}_{j} \subset \mathrm{GL}\left(\Omega^{m-1} \oplus \ldots \oplus \Omega^{m-j-1} \oplus \mathcal{O}^{\oplus j-1}\right)$ the parabolic group subscheme on $X$ preserving for each $1 \leq i \leq j$, the subbundle $\Omega^{m-1} \oplus \ldots \oplus \Omega^{m-i}$. Let $\mathcal{N}_{j} \subset \mathcal{P}_{j}$ be its unipotent radical. Let $M_{j}=\mathcal{P}_{j} / \mathcal{N}_{j}$.

For a finite collection $\underline{y}=\left\{y_{1}, \ldots, y_{r}\right\} \in X$ let $\mathfrak{L}_{\underline{y}}\left(\mathcal{N}_{j}\right)$ and $\mathfrak{L}_{\underline{y}}^{+}\left(\mathcal{N}_{j}\right)$ be the corresponding loop and arc groups as in ([6], 4.4.1). As above, one gets a canonical character

$$
e v_{\underline{\underline{y}}}: \mathfrak{L}_{\underline{\underline{y}}}\left(\mathcal{N}_{j}\right) \rightarrow \prod_{s=1}^{j} \mathfrak{L}_{\underline{\underline{y}}}(\Omega) \rightarrow \mathbb{A}^{1}
$$

where the last map is the sum of residues over all $s$ and all points of $\underline{y}$. The map $e v_{\underline{y}}$ is trivial on $\mathfrak{L}_{\underline{y}}^{+}\left(\mathcal{N}_{j}\right)$. Write $D_{\underline{y}}$ for the formal neighbourhood of $\underline{y}$ in $X$.

Let ${ }_{j+1} \mathcal{Y}_{m \text {, good at } \underline{\underline{Y}}}^{\lambda} \subset_{j+1} \mathcal{Y}_{m}^{\lambda}$ be the open substack given by the property that $\underline{y}$ does not intersect $D_{i}$ for any $i$, and $t_{j+1}$ is a subbundle in a neighbourhood of $\underline{y}$.

For a point of ${ }_{j+1} \mathcal{Y}_{m \text {, good at } \underline{\underline{Y}}}^{\lambda}$ as above, the restriction of $L$ to $D_{\underline{\underline{Y}}}$ naturally gives rise to a $\mathcal{P}_{j}$-torsor on $D_{\underline{\underline{Y}}}$. Moreover, $L / L_{j}$ is equipped with a section $s_{\underline{Y}}: \Omega^{m-j-1} \rightarrow L / L_{j}$ over $D_{\underline{y}}$ such that $t_{j+1}=t_{j} \otimes s_{\underline{Y}}$ over $D_{\underline{Y}}$.

Write $\mathcal{H}\left({ }_{j+1} \mathcal{Y}_{m \text {, good at } \underline{Y}}^{\lambda}\right)$ for the stack classifying a point of ${ }_{j+1} \mathcal{Y}_{m \text {, good at } \underline{Y}}^{\lambda}$ as above together with an isomorphism $L \underset{\rightarrow}{\Im} \Omega^{m-1} \oplus \ldots \oplus \Omega^{m-j} \oplus L / L_{j}$ of $\mathcal{P}_{j}$-torsors over $D_{\underline{y}}$ inducing the identity on the corresponding $M_{j}$-torsors. The projection $\mathcal{H}\left({ }_{j+1} \mathcal{Y}_{m \text {, good at } \underline{\underline{Y}}}^{\lambda}\right) \stackrel{\underline{Y}}{\rightarrow}$ ${ }_{j+1} \mathcal{Y}_{m, \text { good at } \underline{\underline{y}}}^{\lambda}$ is a $\mathfrak{L}_{\underline{\underline{Y}}}^{+}\left(\mathcal{N}_{j}\right)$-torsor.

As in ([6], 4.4.3), the action of $\mathfrak{L}_{\underline{\underline{Y}}}^{+}\left(\mathcal{N}_{j}\right)$ on $\mathcal{H}\left({ }_{j+1} \mathcal{Y}_{m \text {, good at } \underline{\underline{Y}}}^{\lambda}\right)$ naturally extends to a $\mathfrak{L}_{\underline{\underline{y}}}\left(\mathcal{N}_{j}\right)$-action.

Let ${ }_{j} \mathcal{W}_{\text {pos }}^{\lambda}$ be the stack classifying $\left(D_{1}, \ldots, D_{j}\right) \in X_{\text {pos }}^{\lambda}, \bar{L} \in \mathrm{Bun}_{m-j}$ and a section

$$
s: \Omega^{m-j-1} \rightarrow \bar{L}\left(D_{1}+\ldots+D_{j}\right)
$$

We have the projection $\tau_{\text {pos }}^{\lambda}:{ }_{j+1} \mathcal{Y}_{m}^{\lambda} \rightarrow{ }_{j} \mathcal{W}_{\text {pos }}^{\lambda}$ extending $\tau^{\lambda}$. Let ${ }_{j} \mathcal{W}_{\text {pos, good at } \underline{ }}^{\lambda} \subset{ }_{j} \mathcal{W}_{\text {pos }}^{\lambda}$ be the open substack giving by a similar condition: $\underline{y}$ does not intersect $D_{i}$ for all $i$, and $s$ is a subbundle in a neighbourhood of $\underline{y}$. 
The group ind-scheme $\mathfrak{L}_{\underline{\underline{y}}}\left(\mathcal{N}_{j}\right)$ acts transitively on each fibre of the composition

$$
\mathcal{H}\left({ }_{j+1} \mathcal{Y}_{m, \text { good at } \underline{\underline{Y}}}^{\lambda}\right) \rightarrow{ }_{j+1} \mathcal{Y}_{m, \text { good at } \underline{\underline{Y}}}^{\lambda} \stackrel{\tau_{\text {pos }}^{\lambda}}{\rightarrow}{ }_{j} \mathcal{W}_{p o s, \text { good at } \underline{\underline{\prime}}}^{\lambda}
$$

compare with ([6], Lemma 4.4.6). One finishes the proof as in ([6], Sections 4.4 and 4.5).

\subsection{Passing to ${ }^{2 n} \mathcal{Y Z}$}

\section{2 .1}

The purpose of Section 3.2 is to establish Proposition 3.2.3. Its proof after several reductions boils down to a calculation of $\mathrm{R} \Gamma_{c}\left(V, f^{*} \mathcal{L}_{\psi}\right)$ for a finite-dimensional vector space $V$ and a linear morphism $f: V \rightarrow \mathbb{A}^{1}$. A reader may compare with the proof of ([11], Theorem A), which uses a similar strategy in a less involved setting.

\section{2 .2}

Write a point of $\mathcal{Y}_{2 n} \times \mathcal{M}_{2 n} \mathcal{Z}_{n}$ as a collection $L, L^{\prime} \in \operatorname{Bun}_{n}$ for which we set $M=L \oplus L^{\prime}$, and sections

$$
t_{i}: \Omega^{(2 n-1)+\ldots+(2 n-i)} \hookrightarrow \wedge^{i} M
$$

for $1 \leq i \leq 2 n$ satisfying the Plücker relations. Here $t_{1}: \Omega^{2 n-1} \hookrightarrow L$.

For $1 \leq j \leq 2 n$ let

$$
j_{\kappa}:{ }^{j} \mathcal{Y Z} \hookrightarrow \mathcal{Y}_{2 n} \times \mathcal{M}_{2 n} \mathcal{Z}_{n}
$$

be the closed substack given by the following property. For each $1 \leq i \leq j$, we require the following condition (A):

A1) if $i=2 k$ then $t_{2 k}$ factors as

$$
t_{2 k}: \Omega^{(2 n-1)+\ldots+(2 n-2 k)} \hookrightarrow\left(\wedge^{k} L\right) \otimes\left(\wedge^{k} L^{\prime}\right) \subset \wedge^{i} M
$$

A2) if $i=2 k+1$ then $t_{2 k+1}$ factors as

$$
t_{2 k+1}: \Omega^{(2 n-1)+\ldots+(2 n-2 k-1)} \hookrightarrow\left(\wedge^{k+1} L\right) \otimes\left(\wedge^{k} L^{\prime}\right) \subset \wedge^{i} M
$$


We get a diagram of closed embeddings

$$
{ }^{2 n} \mathcal{Y Z} \hookrightarrow \ldots \hookrightarrow{ }^{2} \mathcal{Y} \mathcal{Z} \hookrightarrow{ }^{1} \mathcal{Y} \mathcal{Z}=\mathcal{Y}_{2 n} \times{ }_{\mathcal{M}_{2 n}} \mathcal{Z}_{n}
$$

Our first step is the following.

Proposition 3.2.3. Let $E$ be a local system on $X$. For each $1 \leq j<2 n$, the natural map

$$
\pi_{!} \operatorname{pr}_{2 !}\left({ }_{\kappa}\right)_{*}\left(j_{\kappa}\right)^{*}\left(\mathcal{P}_{2 n, E} \otimes \overline{\mathbb{Q}}_{\ell}\right) \rightarrow \pi_{!} \operatorname{pr}_{2 !}\left({ }^{j+1} \kappa\right)_{*}\left({ }^{j+1} \kappa\right)^{*}\left(\mathcal{P}_{2 n, E} \otimes \overline{\mathbb{Q}}_{\ell}\right)
$$

is an isomorphism.

\subsection{4}

In this subsection, we reduce Proposition 3.2.3 to Proposition 3.2.5. For $1 \leq j \leq 2 n$ let $j \widetilde{\mathcal{Z}}$ be the stack classifying $L, L^{\prime} \in \operatorname{Bun}_{n}$ for which we set $M=L \oplus L^{\prime}$, and sections (2) for $1 \leq i \leq j$ satisfying the Plucker relations such that $t_{1}: \Omega^{2 n-1} \hookrightarrow L \subset M$.

Let ${ }^{j} \mathcal{Z} \hookrightarrow j \widetilde{Z}$ be the closed substack given by the condition (A) on $t_{i}$ for each $1 \leq i \leq j$. Note that ${ }^{1} \mathcal{Z}={ }^{1} \widetilde{\mathcal{Z}}$. Set

$$
{ }^{j+1} \mathcal{Z}^{\prime}={ }^{j+1} \widetilde{\mathcal{Z}} \times{ }_{j} \widetilde{\mathcal{Z}}^{j}{ }
$$

here the projection ${ }^{j+1} \widetilde{\mathcal{Z}} \rightarrow^{j} \widetilde{\mathcal{Z}}$ forgets $t_{j+1}$. For $1 \leq j<2 n$ denote by

$$
j_{\zeta}:{ }^{j} \mathcal{Y Z} \rightarrow{ }^{j+1} \mathcal{Z}^{\prime}
$$

the projection that forgets $t_{j+2}, \ldots, t_{2 n}$. Set

$$
{ }^{j+1} K_{E}={ }^{j} \zeta_{!}\left({ }^{j} \kappa\right) *\left(\mathcal{P}_{2 n, E} \otimes \overline{\mathbb{Q}}_{\ell}\right)
$$

We have a Cartesian square

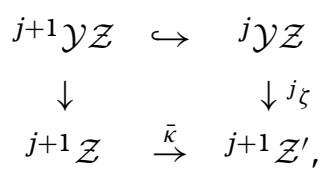

and $\bar{\kappa}$ is a closed immersion. Let $\pi_{\mathcal{Z}}:{ }^{j+1} \mathcal{Z}^{\prime} \rightarrow \operatorname{Pic} X \times \operatorname{Pic} X$ be the map sending a point of ${ }^{j+1} \mathcal{Z}^{\prime}$ to $\left(\operatorname{det} L, \operatorname{det} L^{\prime}\right)$. Proposition 3.2.3 is reduced to the following. 
Proposition 3.2.5. For $1 \leq j<2 n$, the natural map $\left.\pi_{\mathcal{Z} !}{ }^{j+1} K_{E}\right) \rightarrow \pi_{\mathcal{Z} !} \bar{\kappa}_{*} \bar{\kappa}^{*}\left({ }^{j+1} K_{E}\right)$ is an isomorphism.

Our idea of the proof of Proposition 3.2.5 is that it suffices to establish a similar result stratum by stratum after fixing the degrees of zeros of the sections (2) for $1 \leq i \leq j$.

3.2 .6

Our purpose now is to reduce Proposition 3.2.5 to Proposition 3.2.8.

The stack $j \mathcal{Z}$ is stratified by locally closed substacks ${ }^{j} \mathcal{Z}^{\lambda}$ indexed by $\lambda \in \Lambda_{j}^{\succ \text { pos }}$. Let $j=2 k$ for $j$ even (resp., $j=2 k+1$ for $j$ odd). Denote by ${ }^{j} \mathcal{Z}^{\lambda}$ the stack classifying collections:

- $L, L^{\prime} \in \operatorname{Bun}_{n}$ and $\left(D_{1}, \ldots, D_{j}\right) \in X_{p o s}^{\lambda}$ for which we set $M=L \oplus L^{\prime}$;

- a flag of subbundles $\left(0=L_{0}^{\prime} \subset L_{1}^{\prime} \subset \ldots \subset L_{k}^{\prime} \subset L^{\prime}\right)$ together with trivializations

$$
\sigma_{i}^{\prime}: L_{i}^{\prime} / L_{i-1}^{\prime} \stackrel{\sim}{\rightarrow} \Omega^{2 n-2 i}\left(D_{2 i}\right)
$$

for $1 \leq i \leq k$;

- if $j=2 k$ a flag of subbundles $\left(0=L_{0} \subset L_{1} \subset \ldots \subset L_{k} \subset L\right)$ with trivializations

$$
\sigma_{i}: L_{i} / L_{i-1} \stackrel{\widetilde{\rightarrow}}{\Omega^{2 n-2 i+1}}\left(D_{2 i-1}\right)
$$

for $1 \leq i \leq k$;

- if $j=2 k+1$ a flag of subbundles $\left(0=L_{0} \subset L_{1} \subset \ldots \subset L_{k+1} \subset L\right)$ with trivializations (19) for $1 \leq i \leq k+1$.

The locally closed immersion ${ }^{j} \mathcal{Z}^{\lambda} \hookrightarrow{ }^{j} \mathcal{Z}$ is given by the formulas:

- if $1 \leq 2 s \leq j$ then $t_{2 s}=\sigma_{1} \otimes \sigma_{1}^{\prime} \otimes \ldots \otimes \sigma_{s} \otimes \sigma_{s}^{\prime}$ is the map

$$
t_{2 s}: \Omega^{(2 n-1)+\ldots+(2 n-2 s)} \hookrightarrow\left(L_{1} / L_{0}\right) \otimes\left(L_{1}^{\prime} / L_{0}^{\prime}\right) \otimes \ldots \otimes\left(L_{s} / L_{s-1}\right) \otimes\left(L_{s}^{\prime} / L_{s-1}^{\prime}\right) \hookrightarrow \wedge^{2 s} M
$$

- $\quad$ if $1 \leq 2 s+1 \leq j$ then $t_{2 s+1}=\sigma_{1} \otimes \sigma_{1}^{\prime} \otimes \ldots \otimes \sigma_{s+1}$ is the map

$$
t_{2 s+1}: \Omega^{(2 n-1)+\ldots+(2 n-2 s-1)} \hookrightarrow\left(L_{1} / L_{0}\right) \otimes\left(L_{1}^{\prime} / L_{0}^{\prime}\right) \otimes \ldots \otimes\left(L_{s+1} / L_{s}\right) \hookrightarrow \wedge^{2 s+1} M
$$

This stratification is given by fixing the degrees of the divisors of zeros of $t_{i}$ for all $1 \leq i \leq j$. 
Denote by ${ }^{j} \mathcal{Z}_{-}^{\lambda} \hookrightarrow{ }^{j} \mathcal{Z}^{\lambda}$ the closed substack given by $\left(D_{1}, \ldots, D_{j}\right) \in X_{-}^{\lambda}$.

\subsection{7}

For $\lambda \in \Lambda_{j}^{\succ \text { pos }}$ consider the diagram

$$
\begin{array}{rcc}
{ }^{j+1} \mathcal{Z} \times_{j} \mathcal{Z}^{j} \mathcal{Z}^{\lambda} \stackrel{\bar{\kappa}^{\lambda}}{\rightarrow} & { }^{j+1} \mathcal{Z}^{\prime} \times_{j} \mathcal{Z}^{j} \mathcal{Z}^{\lambda} \\
& \searrow & \downarrow \pi_{\mathcal{Z}}^{\lambda} \\
& & \operatorname{Pic} X \times \operatorname{Pic} X,
\end{array}
$$

where $\bar{\kappa}^{\lambda}$ is the restriction of $\bar{\kappa}$, and $\pi_{\mathcal{Z}}^{\lambda}$ is the restriction of $\pi_{\mathcal{Z}}$. Note that $\bar{\kappa}^{\lambda}$ is a closed embedding.

Denote by ${ }^{j+1} K_{E}^{\lambda}$ the $*$-restriction of ${ }^{j+1} K_{E}$ to ${ }^{j+1} \mathcal{Z}^{\prime} \times_{j} \mathcal{Z}^{j} \mathcal{Z}^{\lambda}$. By Proposition 3.1.3, the $*$-restriction of $\mathcal{P}_{2 n, E}^{d}$ to $S(\mathcal{Y})_{\text {pos }}^{v}$ vanishes for all but a finite number of these strata for $v \in \Lambda_{2 n, d}^{>\text {pos }}$. This reduces Proposition 3.2.5 to the following.

Proposition 3.2.8. For $\lambda \in \Lambda_{j}^{\succ \text { pos }}$, the natural map $\left.\left(\pi_{\mathcal{Z}}^{\lambda}\right)_{!}{ }^{j+1} K_{E}^{\lambda}\right) \rightarrow\left(\pi_{\mathcal{Z}}^{\lambda}\right)_{!} \bar{\kappa}_{*}^{\lambda}\left(\bar{\kappa}^{\lambda}\right)^{*}\left({ }^{j+1} K_{E}^{\lambda}\right)$ is an isomorphism.

\subsubsection{Proof of Proposition 3.2.8}

Let $j=2 k$ for $j$ even (resp., $j=2 k+1$ for $j$ odd). The stack ${ }^{j+1} \mathcal{Z}^{\prime} \times_{j}{ }^{j} \mathcal{Z}^{\lambda}$ classifies collections: a point of ${ }^{j} \mathcal{Z}^{\lambda}$ as described in Section 3.2.6,

- if $j=2 k$ then we are also given a section

$$
s: \Omega^{2 n-2 k-1} \rightarrow\left(M /\left(L_{k} \oplus L_{k}^{\prime}\right)\right)\left(D_{1}+\ldots+D_{2 k}\right),
$$

for which we define $t_{j+1}$ as the map

$$
t_{j} \otimes s: \Omega^{(2 n-1)+\ldots+(2 n-2 k-1)} \rightarrow \operatorname{det}\left(L_{k} \oplus L_{k}^{\prime}\right) \otimes\left(M /\left(L_{k} \oplus L_{k}^{\prime}\right)\right) \subset \wedge^{j+1} M
$$

- if $j=2 k+1$ then we are given a section

$$
s: \Omega^{2 n-2 k-2} \rightarrow\left(M /\left(L_{k+1} \oplus L_{k}^{\prime}\right)\right)\left(D_{1}+\ldots+D_{2 k+1}\right),
$$

and we define $t_{j+1}$ as the map

$$
t_{j} \otimes s: \Omega^{(2 n-1)+\ldots+(2 n-2 k-2)} \rightarrow \operatorname{det}\left(L_{k+1} \oplus L_{k}^{\prime}\right) \otimes\left(M /\left(L_{k+1} \oplus L_{k}^{\prime}\right)\right) \subset \wedge^{j+1} M
$$


Let

$$
{ }^{j+1} \overline{\mathcal{Z}}^{\prime \lambda}{ }^{j+1} \mathcal{Z}^{\prime} \times_{j} \mathcal{Z}^{j} \mathcal{Z}^{\lambda}
$$

be the closed substack given by the properties that $D_{1} \leq \ldots \leq D_{j}$, and

- if $j=2 k$ then $s: \Omega^{2 n-2 k-1}\left(D_{2 k}\right) \rightarrow M /\left(L_{k} \oplus L_{k}^{\prime}\right)$ is regular

- if $j=2 k+1$ then $s: \Omega^{2 n-2 k-2}\left(D_{2 k+1}\right) \rightarrow M /\left(L_{k+1} \oplus L_{k}^{\prime}\right)$ is regular.

By Lemma 3.1.5, one knows that ${ }^{j+1} K_{E}^{\lambda}$ is the extension by zero from ${ }^{j+1} \overline{\mathcal{Z}}^{\prime \lambda}$. Let ${ }^{j+1} \overline{\mathcal{Z}}^{\lambda}$ be the preimage of ${ }^{j+1} \overline{\mathcal{Z}}^{\prime \lambda}$ under $\bar{\kappa}^{\lambda}$. So, in Proposition 3.2.8, we actually deal with the diagram

$$
\begin{array}{rcc}
j+1 \overline{\mathcal{Z}}^{\lambda} \stackrel{\bar{\kappa}^{\lambda}}{\rightarrow} & j+1 \overline{\mathcal{Z}}^{\prime \lambda} \\
& \searrow & \downarrow \pi_{\mathcal{Z}}^{\lambda} \\
& & \operatorname{Pic} X \times \operatorname{Pic} X
\end{array}
$$

By abuse of notations, here $\pi_{\mathcal{Z}}^{\lambda}$ and $\bar{\kappa}^{\lambda}$ denote the restrictions of the corresponding maps. The description of ${ }^{j+1} K_{E}^{\lambda}$ on ${ }^{j+1} \overline{\mathcal{Z}}^{\prime \lambda}$ is as follows. Let ${ }^{j+1} \overline{\mathcal{W}}^{\lambda}$ be the stack classifying $\left(D_{1}, \ldots, D_{j}\right) \in X_{-}^{\lambda}$,

- $\quad$ if $j=2 k$ then we are given $\bar{L}, \bar{L}^{\prime} \in \operatorname{Bun}_{n-k}$ and $s: \Omega^{2 n-2 k-1}\left(D_{2 k}\right) \hookrightarrow \bar{L} \oplus \bar{L}^{\prime}$;

- if $j=2 k+1$ then we are given $\bar{L} \in \operatorname{Bun}_{n-k-1}, \bar{L}^{\prime} \in \operatorname{Bun}_{n-k}$ and

$$
s: \Omega^{2 n-2 k-2}\left(D_{2 k+1}\right) \hookrightarrow \bar{L} \oplus \bar{L}^{\prime}
$$

We get a map $\bar{\tau}:{ }^{j+1} \overline{\mathcal{Z}}^{\prime \lambda} \rightarrow{ }^{j+1} \overline{\mathcal{W}}^{\lambda}$ given by the formulas $\bar{L}^{\prime}=L^{\prime} / L_{k}^{\prime}$ and

$$
\bar{L}= \begin{cases}L / L_{k}, & \text { if } j=2 k \\ L / L_{k+1}, & \text { if } j=2 k+1\end{cases}
$$

Let $e v^{\lambda}:{ }^{j+1} \overline{\mathcal{Z}}^{\prime \lambda} \rightarrow \mathbb{A}^{1}$ be the map sending a point of ${ }^{j+1} \overline{\mathcal{Z}}^{\prime \lambda}$ to the class of the pullback extension

$$
\begin{aligned}
& 0 \rightarrow L_{k}^{\prime} / L_{k-1}^{\prime} \rightarrow L^{\prime} / L_{k-1}^{\prime} \rightarrow \quad L^{\prime} / L_{k}^{\prime} \quad \rightarrow 0 \\
& \uparrow \bar{s}^{\prime} \\
& \Omega^{2 n-2 k-1}\left(D_{2 k}\right)
\end{aligned}
$$


for $j=2 k$, and the class of the pullback extension

$$
\begin{aligned}
& 0 \rightarrow L_{k+1} / L_{k} \rightarrow L / L_{k} \rightarrow \begin{array}{c}
L / L_{k+1} \\
\uparrow \bar{s}
\end{array} \\
& \\
& \Omega^{2 n-2 k-2}\left(D_{2 k+1}\right)
\end{aligned}
$$

for $j=2 k+1$, respectively. Here, $\bar{s}, \bar{s}^{\prime}$ are the corresponding components of $s$.

Lemma 3.2.10. There is a complex ${ }^{j+1} \bar{K}_{E}^{\lambda}$ on ${ }^{j+1} \overline{\mathcal{W}}^{\lambda}$ and an isomorphism over ${ }^{j+1} \overline{\mathcal{Z}}^{\prime \lambda}$

$$
{ }^{j+1} K_{E}^{\lambda} \underset{\rightarrow}{\rightarrow} \bar{\tau}^{*}\left({ }^{j+1} \bar{K}_{E}^{\lambda}\right) \otimes\left(e v^{\lambda}\right)^{*} \mathcal{L}_{\psi}
$$

Proof. This follows from Lemma 3.1.5. In more details, recall the stack ${ }_{j+1} \mathcal{Y}_{2 n_{-}-}^{\lambda}$ from Section 3.1 .4 . We have the map ${ }^{j+1} \overline{\mathcal{Z}}^{\prime \lambda} \rightarrow{ }_{j+1} \mathcal{Y}_{2 n,-}^{\lambda}$ sending a point of the source to the collection

$$
\left(M=L \oplus L^{\prime},\left(t_{1}, \ldots, t_{j+1}\right),\left(D_{1}, \ldots, D_{j}\right) \in X_{-}^{\lambda}\right)
$$

So, $M$ is equipped with the flag $0=M_{0} \subset M_{1} \subset \ldots \subset M_{j} \subset M$ of subbundles. Here $M_{2 k}=$ $L_{k} \oplus L_{k}^{\prime}$ for $0 \leq 2 k \leq j$ and $M_{2 k+1}=L_{k+1} \oplus L_{k}^{\prime}$ for $0<2 k+1 \leq j$. Our claim follows from the fact that, by construction, the exact sequence $0 \rightarrow M_{i} / M_{i-1} \rightarrow M_{i+1} / M_{i} \rightarrow M_{i+1} / M_{i} \rightarrow 0$ splits canonically for $1 \leq i \leq j-1$.

The $\operatorname{map} \pi_{\mathcal{Z}}^{\lambda}$ factors as

$$
{ }^{j+1} \overline{\mathcal{Z}}^{\prime \lambda} \stackrel{\bar{\tau}}{\rightarrow}{ }^{j+1} \overline{\mathcal{W}}^{\lambda} \rightarrow \operatorname{Pic} X \times \operatorname{Pic} X
$$

Let $^{j+1} \mathcal{W}^{\lambda} \hookrightarrow{ }^{j+1} \overline{\mathcal{W}}^{\lambda}$ be the closed substack given by the property

- if $j=2 k$ then $s$ factors as $s: \Omega^{(2 n-2 k-1)}\left(D_{2 k}\right) \hookrightarrow \bar{L} \subset \bar{L} \oplus \bar{L}^{\prime}$;

- if $j=2 k+1$ then $s$ factors as $s: \Omega^{(2 n-2 k-2)}\left(D_{2 k+1}\right) \hookrightarrow \bar{L}^{\prime} \subset \bar{L} \oplus \bar{L}^{\prime}$.

The square is Cartesian

$$
\begin{array}{ccc}
{ }^{j+1} \mathcal{W}^{\lambda} & \hookrightarrow & { }^{j+1} \overline{\mathcal{W}}^{\lambda} \\
\uparrow & & \uparrow \bar{\tau} \\
{ }^{j+1} \overline{\mathcal{Z}}^{\lambda} & \stackrel{\bar{\kappa}^{\lambda}}{\rightarrow} & { }^{j+1} \overline{\mathcal{Z}}^{\prime \lambda}
\end{array}
$$


So, it suffices to show that $\bar{\tau}_{1}\left(e v^{\lambda}\right)^{*} \mathcal{L}_{\psi}$ is the extension by zero from ${ }^{j+1} \mathcal{W}^{\lambda}$. The map $\bar{\tau}$ is a generalized affine fibration. Let ${ }^{j+1} \hat{\mathcal{W}}^{\lambda}$ be the stack classifying a point of ${ }^{j+1} \overline{\mathcal{W}}^{\lambda}$ as above together with an exact sequence

$$
0 \rightarrow \Omega^{2 n-2 k}\left(D_{2 k}\right) \rightarrow ? \rightarrow \bar{L}^{\prime} \rightarrow 0
$$

for $j=2 k$, respectively, with an exact sequence

$$
0 \rightarrow \Omega^{2 n-2 k-1}\left(D_{2 k+1}\right) \rightarrow ? \rightarrow \bar{L} \rightarrow 0
$$

for $j=2 k+1$. The map $\bar{\tau}$ decomposes naturally as

$$
{ }^{j+1} \overline{\mathcal{Z}}^{\prime \lambda} \stackrel{\xi}{\rightarrow}{ }^{j+1} \hat{\mathcal{W}}^{\lambda} \stackrel{\hat{\tau}}{\rightarrow}{ }^{j+1} \overline{\mathcal{W}}^{\lambda},
$$

where $\xi$ is a generalized affine fibration. Namely, the sequence (21) for $j=2 k$ is

$$
0 \rightarrow L_{k}^{\prime} / L_{k-1}^{\prime} \rightarrow L^{\prime} / L_{k-1}^{\prime} \rightarrow L^{\prime} / L_{k}^{\prime} \rightarrow 0
$$

The sequence (22) for $j=2 k+1$ is

$$
0 \rightarrow L_{k+1} / L_{k} \rightarrow L / L_{k} \rightarrow L / L_{k+1} \rightarrow 0
$$

Here $\hat{\tau}$ forgets the extension (21) for $j=2 k$ (respectively, the extension (22) for $j=2 k+1$ ). The map $e V^{\lambda}$ factors naturally as ${ }^{j+1} \overline{\mathcal{Z}}^{\prime \lambda} \rightarrow^{j+1} \hat{\mathcal{W}}^{\lambda} \stackrel{\hat{e V}^{\lambda}}{\rightarrow} \mathbb{A}^{1}$, and $\xi_{!} \overline{\mathbb{Q}}_{\ell}$ identifies with $\overline{\mathbb{Q}}_{\ell}$ up to a shift. The $*$-fibre of $\hat{t}_{!}\left(\hat{e V^{\lambda}}\right)^{*} \mathcal{L}_{\psi}$ at a given point vanishes unless $\bar{s}^{\prime}=0$ for $j=2 k$ (respectively, $\bar{s}=0$ for $j=2 k+1$ ). Proposition 3.2.8 is proved.

Thus, Propositions 3.2.5 and 3.2.3 are also proved.

\subsection{Passing to $\tilde{\mathcal{Y}}_{n} \times \tilde{\mathcal{Y}}_{n}^{\prime}$}

\subsection{1}

In Section 3.3, we finish the proof of Proposition 2.3.4 using the results of Sections 3.1 and 3.2 . 


\subsection{2}

Note that ${ }^{2 n} \mathcal{Z}={ }^{2 n} \mathcal{Y Z}$. For $d, d^{\prime} \in \mathbb{Z}$ let ${ }^{2 n} \mathcal{Z}^{d, d^{\prime}}$ be the substack of ${ }^{2 n} \mathcal{Z}$ given by

$$
\operatorname{deg} L=\operatorname{deg}\left(\Omega^{(2 n-1)+(2 n-3)+\ldots+1}\right)+d, \operatorname{deg} L^{\prime}=\operatorname{deg}\left(\Omega^{(2 n-2)+(2 n-4)+\ldots+2}\right)+d^{\prime}
$$

The map $q_{\mathcal{Y Z}}: \tilde{\mathcal{Y}}_{n, d} \times \tilde{\mathcal{Y}}_{n, d^{\prime}}^{\prime} \rightarrow \mathcal{Y}_{2 n} \times \mathcal{M}_{2 n} \mathcal{Z}_{n}$ factors uniquely through the closed substack

$$
\tilde{\mathcal{Y}}_{n, d} \times \tilde{\mathcal{Y}}_{n, d^{\prime}}^{\prime} \stackrel{i_{\mathcal{Y}}}{\rightarrow} 2 n_{\mathcal{Z}} \mathcal{d , d}^{\prime} \stackrel{2 n_{\kappa}}{\hookrightarrow} \mathcal{Y}_{2 n} \times{ }_{\mathcal{M}_{2 n}} \mathcal{Z}_{n^{\prime}}
$$

this defines the map $i_{\mathcal{Y}}$.

\subsection{3}

The stack ${ }^{2 n} \mathcal{Z}$ admits a stratification by ${ }^{2 n} \mathcal{Z}^{\lambda}$ indexed by $\lambda \in \Lambda_{2 n}^{>\text {pos }}$, which is described in Section 3.2.6. For $d, d^{\prime} \in \mathbb{Z}$ the stack ${ }^{2 n} \mathcal{Z}^{d, d^{\prime}}$ admits a stratification by ${ }^{2 n} \mathcal{Z}^{\lambda}$ indexed by $\lambda \in \Lambda_{2 n}^{\succ \text { pos }}$ such that $\lambda_{1}+\lambda_{3}+\ldots+\lambda_{2 n-1}=d$ and $\lambda_{2}+\ldots+\lambda_{2 n}=d^{\prime}$.

For $\lambda \in \Lambda_{2 n}^{\succ-}$ let

$$
q_{\lambda}:{ }^{2 n} \mathcal{Z}_{-}^{\lambda} \rightarrow X_{-}^{\lambda}
$$

be the map sending a point of ${ }^{2 n} \mathcal{Z}_{-}^{\lambda}$ to $\left(D_{1}, \ldots, D_{2 n}\right) \in X_{-}^{\lambda}$. From Proposition 3.1.3, one gets the following.

Corollary 3.3.4. Let $\lambda \in \Lambda_{2 n, r}^{\succ \text { pos }}$. The $*$-restriction of $\left(2{ }^{2 n} \kappa\right)^{*}\left(\mathcal{P}_{2 n, E}^{r} \otimes \overline{\mathbb{Q}}_{\ell}\right)$ to the stratum ${ }^{2 n} \mathcal{Z}^{\lambda}$ vanishes unless $\lambda \in \Lambda_{2 n, r}^{\succ-}$. In the latter case, it is the extension by zero from ${ }^{2 n} \mathcal{Z}_{-}^{\lambda}$ and identifies over ${ }^{2 n} \mathcal{Z}_{-}^{\lambda}$ with

$$
q_{\lambda}^{*} E_{-}^{\lambda}\left[b(2 n, r)-2 a_{2 n}(\lambda)\right]
$$

\subsection{5}

For a scheme $S$ and a vector bundle $V$ on $S \times X$, by a rational section of $V$ we mean a section defined over an open subscheme of the form $(S \times X)-D$, where $D \hookrightarrow S \times X$ is a closed subscheme finite over $S$.

Lemma 3.3.6. A point of ${ }^{2 n} \mathcal{Z}$ gives rise to uniquely defined rational sections

$$
s_{i}: \Omega^{(2 n-1)+\ldots+(2 n-2 i+1)} \hookrightarrow \wedge^{i} L
$$


and

$$
s_{i}^{\prime}: \Omega^{(2 n-2)+\ldots+(2 n-2 i)} \hookrightarrow \wedge^{i} L^{\prime}
$$

for $1 \leq i \leq n$ such that (10) hold.

Proof. A point of of ${ }^{2 n} \mathcal{Z}$ gives rise to nonuniquely defined rational sections $u_{i}$ : $\Omega^{2 n-2 i+1} \hookrightarrow L$ and $u_{i}^{\prime}: \Omega^{2 n-2 i} \hookrightarrow L^{\prime}$ for $1 \leq i \leq n$ such that $t_{2 k}=u_{1} \wedge u_{1}^{\prime} \wedge \ldots \wedge u_{k} \wedge u_{k}^{\prime}$ and

$$
t_{2 k+1}=u_{1} \wedge u_{1}^{\prime} \wedge \ldots \wedge u_{k} \wedge u_{k}^{\prime} \wedge u_{k+1}
$$

Set $s_{k}=u_{1} \wedge \ldots \wedge u_{k}$ and $s_{k}^{\prime}=u_{1}^{\prime} \wedge \ldots \wedge u_{k}^{\prime}$. One checks that $s_{k}$ and $s_{k}^{\prime}$ are independent of the choices of $u_{i}, u_{i}^{\prime}$.

\subsubsection{Proof of Proposition 2.3.4}

From Lemma 3.3.6, it follows that $i_{\mathcal{Y}}$ is a closed immersion, and it is given by the property that the rational sections (23) and (24) are regular over the whole of $X$.

More precisely, this is seen as follows. For a point $\left(L, L^{\prime},\left(t_{i}\right)\right) \in{ }^{2 n} \mathcal{Z}$ consider the exact sequence $0 \rightarrow \Omega^{2 n-1} \stackrel{t_{1}}{\rightarrow} L \rightarrow L / \Omega^{2 n-1} \rightarrow 0$. Set $s_{1}=t_{1}$. As the first step, one imposes the condition that the image of $t_{2}$ under the induced map

$$
L \otimes L^{\prime} \rightarrow\left(L / \Omega^{2 n-1}\right) \otimes L^{\prime}
$$

vanishes, this yields a section $s_{1}^{\prime}: \Omega^{2 n-2} \hookrightarrow L^{\prime}$ with $t_{2}=s_{1} \otimes s_{1}^{\prime}$. Consider the exact sequence $0 \rightarrow \Omega^{2 n-2} \stackrel{s_{1}^{\prime}}{\rightarrow} L^{\prime} \rightarrow L^{\prime} / \Omega^{2 n-2} \rightarrow 0$. As the second step, one imposes the condition that the image of $t_{3}$ under the induced map

$$
\left(\wedge^{2} L\right) \otimes L^{\prime} \rightarrow\left(\wedge^{2} L\right) \otimes\left(L^{\prime} / \Omega^{2 n-2}\right)
$$

vanishes. This yields a section $s_{2}: \Omega^{(2 n-1)+(2 n-3)} \hookrightarrow \wedge^{2} L$ such that $t_{3}=-s_{2} \otimes s_{1}^{\prime}$. Continuing this procedure, by induction one gets all the sections $s_{i}, s_{i}^{\prime}$ by imposing similar closed conditions.

From Corollary 3.3.4, it follows that $\left({ }^{2 n} \kappa\right)^{*}\left(\mathcal{P}_{2 n, E} \otimes \overline{\mathbb{Q}}_{\ell}\right)$ over the component ${ }^{2 n} \mathcal{Z}^{d, d^{\prime}}$ of ${ }^{2 n} \mathcal{Z}$ is the extension by zero under the map $i_{\mathcal{Y}}$. Indeed, for each $\lambda \in \Lambda_{2 n}^{\succ \text { pos }}$ such that 
$\lambda_{1}+\lambda_{3}+\ldots+\lambda_{2 n-1}=d$ and $\lambda_{2}+\ldots+\lambda_{2 n}=d^{\prime}$, we have

$$
\left.{ }^{2 n} \mathcal{Z}_{-}^{\lambda} \subset\left(\tilde{\mathcal{Y}}_{n, d} \times \tilde{\mathcal{Y}}_{n, d^{\prime}}^{\prime}\right) \times{ }_{(2 n} \mathcal{Z}^{d, d^{\prime}}\right){ }^{2 n} \mathcal{Z}^{\lambda}
$$

Indeed, if a point of ${ }^{2 n} \mathcal{Z}_{-}^{\lambda}$ is written as in Section 3.2.6, then the divisor of zeros of (23) is $D_{1}+D_{3}+\ldots+D_{2 i-1} \geq 0$ and the divisor of zeros of (24) is $D_{2}+D_{4}+\ldots D_{2 i} \geq 0$. Our claim follows now from Proposition 3.2.3.

Remark 3.3.8. The map $i_{\mathcal{Y}}: \widetilde{\mathcal{Y}}_{n, d} \times \widetilde{\mathcal{Y}}_{n, d^{\prime}}^{\prime} \rightarrow{ }^{2 n} \mathcal{Z}^{d, d^{\prime}}$ is not an isomorphism already for $n=1$. In this case, the condition (A) is empty, and ${ }^{2} \mathcal{Z}^{d, d^{\prime}}$ classifies: $L, L^{\prime} \in$ Bun $_{1}$ with $\operatorname{deg} L=\operatorname{deg} \Omega+d, \operatorname{deg} L^{\prime}=d^{\prime}$ and sections

$$
t_{1}: \Omega \hookrightarrow L, t_{2}: \Omega \hookrightarrow L \otimes L^{\prime}
$$

The closed immersion $i_{\mathcal{Y}}$ is given in this case by requiring that there is $s_{1}^{\prime}: \mathcal{O} \hookrightarrow L^{\prime}$ such that $t_{2}=t_{1} \otimes s_{1}^{\prime}$.

\section{Direct image under $\bar{\pi}$}

The purpose of Section 4 is to prove Proposition 2.3.5, which is a part of our strategy presented in Section 2.3. We start with Proposition 4.1.2 showing that the complex (13) that we must calculate is placed in the highest usual cohomological degree only. It also provides a filtration on the corresponding constructible sheaf whose associated graded is explicitly described. The corresponding highest direct image is calculated in two steps.

The first step is Proposition 4.2.4. Namely, we get a diagram

$$
\widetilde{\mathcal{Q}}_{n, d} \times \widetilde{\mathcal{Q}}_{n, d^{\prime}}^{\prime} \stackrel{\tilde{\varphi} \times \tilde{\varphi}^{\prime}}{\rightarrow} \widetilde{\mathcal{Y}}_{n, d} \times \widetilde{\mathcal{Y}}_{n, d^{\prime}}^{\prime} \stackrel{\bar{\pi}}{\rightarrow} X^{(d)} \times X^{\left(d^{\prime}\right)}
$$

and replace the highest direct image under $\bar{\pi}$ by the highest direct image under $\bar{\pi} \circ$ $\left(\tilde{\varphi} \times \tilde{\varphi}^{\prime}\right)$. This is obtained by a reduction to some local statement with respect to $X$. We generalize the corresponding local result for an arbitrary reductive group and a Levi subgroup that we call antistandard. This generalization is performed in Appendix A independent of the rest of the paper. We consider this generalization as an important result of independent interest (Propositions A.0.5 and A.2.2). 
The second step is Proposition 4.3.2. The combination of both steps easily implies Proposition 2.3.5. In turn, Proposition 4.3.2 is reduced to Theorem 4.3.9, whose proof is the purpose of Section 4.4 .

The mathematical content of Proposition 4.3.2 and Theorem 4.3.9 is to decompose $\bar{\pi} \circ\left(\tilde{\varphi} \times \tilde{\varphi}^{\prime}\right)$ as

$$
\widetilde{\mathcal{Q}}_{n, d} \times \widetilde{\mathcal{Q}}_{n, d^{\prime}}^{\prime} \stackrel{\tilde{\beta} \times \tilde{\beta}^{\prime}}{\rightarrow} \leq n \operatorname{Sh}_{0}^{d} \times \leq n \operatorname{Sh}_{0}^{d^{\prime}} \stackrel{\leq n}{\stackrel{\operatorname{div} \times \operatorname{div})}{\rightarrow}} X^{(d)} \times X^{\left(d^{\prime}\right)}
$$

and reduce the calculation of the desired highest direct image under $\bar{\pi} \circ\left(\tilde{\varphi} \times \tilde{\varphi}^{\prime}\right)$ to the calculation of the highest direct image under $\leq n$ (div $\times$ div) over the closed subscheme $i_{X}: X^{(d)} \times X^{\left(d^{\prime}-d\right)} \hookrightarrow X^{(d)} \times X^{\left(d^{\prime}\right)}$ given by (6). The last calculation given in Theorem 4.3.9 is of local nature with respect to $X$. We consider Theorem 4.3 .9 as one of our main results, which is of independent interest.

\subsection{Filtration}

In Section 4.1, we prove Proposition 4.1.2. It shows that (13) is a constructible sheaf (up to a shift) and calculates this sheaf in the Grothendieck group.

\subsection{1}

Recall the map (6) from Section 2.1.1. From Corollary 3.3.4, it follows that

$$
\bar{\pi}_{!} q_{\mathcal{Y} Z}^{*}\left(\mathcal{P}_{2 n, E} \otimes \overline{\mathbb{Q}}_{\ell}\right)
$$

is the extension by zero under $i_{X}$. In particular, it vanishes unless $0 \leq d \leq d^{\prime}$. Indeed, this holds for the contribution of each stratum ${ }^{2 n} \mathcal{Z}^{\lambda}$ of ${ }^{2 n} \mathcal{Z}^{d, d^{\prime}}$.

For $\lambda \in \Lambda_{2 n}^{\succ \text { pos }}$ recall the notation $\lambda^{\text {odd }}, \lambda^{\text {even }}$ and the map $\operatorname{sum}^{\lambda}: X_{-}^{\lambda} \rightarrow X^{(d)} \times X^{\left(d^{\prime}\right)}$ from Sections 2.4.1 and 2.4.3.

Proposition 4.1.2. The complex

$$
\bar{\pi}_{!} q_{\mathcal{Y} \mathcal{Z}}^{*}\left(\mathcal{P}_{2 n, E}^{d+d^{\prime}} \otimes \overline{\mathbb{Q}}_{\ell}\right)\left[\operatorname{dim} \cdot \operatorname{rel}\left(v_{\mathcal{Z}}\right)-d^{\prime}\right]
$$

on $X^{(d)} \times X^{\left(d^{\prime}\right)}$ is a constructible sheaf placed in usual cohomological degree zero, which is an extension by zero under $i_{X}$. It admits a filtration with the associated graded

$$
\underset{\lambda}{\oplus} \operatorname{sum}_{!}^{\lambda} E_{-}^{\lambda}
$$


the sum being taken over $\lambda \in \Lambda_{2 n, d+d^{\prime}}^{\succ-}$ such that $\lambda^{\text {odd }} \in \Lambda_{n, d}^{\succ-}$ and $\lambda^{\text {even }} \in \Lambda_{n, d^{\prime}}^{\succ-}$.

Proof. For each $\lambda \in \Lambda_{2 n}^{\succ \text { pos }}$ the projection ${ }^{2 n} \mathcal{Z}^{\lambda} \rightarrow X_{\text {pos }}^{\lambda}$ is a generalized affine fibration of rank

$$
n d+(n-1) d^{\prime}-a_{2 n}(\lambda)-n(n-1)(g-1)-(4 g-4) \sum_{i=1}^{n-1} i^{2}
$$

The same holds for $q_{\lambda}:{ }^{2 n} \mathcal{Z}_{-}^{\lambda} \rightarrow X_{-}^{\lambda}$. Since $\sum_{i=1}^{n} i^{2}=\frac{n}{6}(n+1)(2 n+1)$, from (15) one gets

$$
b\left(2 n, d+d^{\prime}\right)=2 n\left(d+d^{\prime}\right)+(1-g) \frac{(2 n-1)}{3} n(4 n-1)
$$

Using Corollary 3.3.4, calculate (25) with respect to the stratification of ${ }^{2 n} \mathcal{Z}^{d, d^{\prime}}$ by the substacks ${ }^{2 n} \mathcal{Z}^{\lambda}$. The strata that contribute are ${ }^{2 n} \mathcal{Z}_{-}^{\lambda}$ for $\lambda \in \Lambda_{2 n, d+d^{\prime}}^{\succ-}$ such that $\lambda^{\text {odd }} \in \Lambda_{n, d}^{\succ-}$ and $\lambda^{\text {even }} \in \Lambda_{n, d^{\prime}}^{\succ-}$. For such $\lambda$, we get

$$
q_{\lambda !} q_{\lambda}^{*} E_{-}^{\lambda}\left[b\left(2 n, d+d^{\prime}\right)-2 a_{2 n}(\lambda)\right] \stackrel{\rightarrow}{\rightarrow} E_{-}^{\lambda}\left[2 d^{\prime}+(1-g) n\right]
$$

the shift in the right hand side is independent of $\lambda$.

Now calculate the relative dimension of $v_{\mathcal{Z}}$. One has $\operatorname{dim}\left(\mathcal{M}_{2 n, r}\right)=r$. Let $\mathcal{Z}_{n}^{d, d^{\prime}}$ be the component of $\mathcal{Z}_{n}$ given by

$$
\operatorname{deg} L=d+\operatorname{deg}\left(\Omega^{(2 n-1)+(2 n-3)+\ldots+1}\right), \quad \operatorname{deg} L^{\prime}=d^{\prime}+\operatorname{deg}\left(\Omega^{(2 n-2)+(2 n-4)+\ldots+0}\right)
$$

Since $\operatorname{dim} \mathcal{Z}_{n}^{d, d^{\prime}}=d+n(g-1)$, for

$$
v_{\mathcal{Z}}: \mathcal{Z}_{n}^{d, d^{\prime}} \rightarrow \mathcal{M}_{2 n, d+d^{\prime}}
$$

we get $\operatorname{dim} . \operatorname{rel}\left(v_{\mathcal{Z}}\right)=n(g-1)-d^{\prime}$. So, the contribution of ${ }^{2 n} \mathcal{Z}^{\lambda}$ to (25) is sum! $E_{-}^{\lambda}$.

Remark 4.1.3. i) In the case $d=0$ the filtration of Proposition 4.1.2 on (25) has only one term, and Proposition 2.3.5 follows from Proposition 4.1.2. Indeed, for $\lambda=$ $\left(0, \ldots, 0, d^{\prime}\right)$ one has $E_{-}^{\lambda}=E^{\left(d^{\prime}\right)}$ on $X_{-}^{\lambda}=X^{\left(d^{\prime}\right)}$.

ii) In view of Proposition 4.1.2, to prove Proposition 2.3.5 for $\operatorname{rk}(E)=2 n$, it suffices to show that the left hand side of (13) is the intermediate extension of its restriction to any nonempty open subscheme of $X^{(d)} \times X^{\left(d^{\prime}-d\right)}$. Indeed, if $\left(D, D^{\prime}\right) \in X^{(d)} \times X^{\left(d^{\prime}\right)}$ with $D^{\prime} \geq D$, $D$ is reduced, $D^{\prime}-D$ is reduced, and $D \cap\left(D^{\prime}-D\right)=\emptyset$ then only one $\lambda$ as in Proposition 
4.1.2 contributes, namely $\lambda=\left(0, \ldots, 0, d, d^{\prime}\right)$. The restriction of $E_{-}^{\lambda}$ to the corresponding open subscheme of

$$
i_{X}\left(X^{(d)} \times X^{\left(d^{\prime}-d\right)}\right)
$$

identifies with $\left(\wedge^{2} E\right)^{(d)} \otimes E^{\left(d^{\prime}-d\right)}$.

\subsection{Highest direct image: first step}

The purpose of Section 4.2 is to prove Proposition 4.2.4. The plan of its proof is as follows. Stratifying the stack $\widetilde{\mathcal{Y}}_{n, d} \times \widetilde{\mathcal{Y}}_{n, d^{\prime}}^{\prime}$ naturally, our claim is easily reduced to Proposition 4.2.7. The latter, in turn, is easily reduced to Proposition 4.2.7, which is of local nature. Finally, Proposition 4.2.10 is obtained as a particular case of a more general Proposition A.2.2 valid for any reductive group $G$ and its antistandard Levi subgroup $M$. The latter is established in Appendix A independent of the rest of the paper.

\section{2 .1}

For $d \geq 0$ let $\widetilde{\mathcal{Q}}_{n, d}$ be the stack classifying: a modification of rank $n$ vector bundles $L_{n} \subset L$ on $X$ with $\operatorname{deg}\left(L / L_{n}\right)=d$, a complete flag of vector subbundles $0=L_{1} \subset \ldots \subset L_{n}$, where $L_{i}$ is a rank $i$ vector bundle, and isomorphisms

$$
\sigma_{i}: L_{i} / L_{i-1} \stackrel{\sim}{\rightarrow} \Omega^{2 n-2 i+1}
$$

for $1 \leq i \leq n$. We have a morphism $\tilde{\varphi}: \widetilde{\mathcal{Q}}_{n, d} \rightarrow \widetilde{\mathcal{Y}}_{n, d}$ sending the above point to $\left(L,\left(s_{i}\right)\right)$, where

$$
s_{i}: \Omega^{(2 n-1)+\ldots+(2 n-2 i+1)} \underset{\rightarrow}{\sim} \wedge^{i} L_{i} \hookrightarrow \wedge^{i} L
$$

for $1 \leq i \leq n$.

Similarly, for $d \geq 0$, let $\widetilde{\mathcal{Q}}_{n, d}^{\prime}$ be the stack classifying: a modification of rank $n$ vector bundles $L_{n}^{\prime} \subset L^{\prime}$ on $X$ with $\operatorname{deg}\left(L^{\prime} / L_{n}^{\prime}\right)=d$, a complete flag $\left(0=L_{0}^{\prime} \subset L_{1}^{\prime} \subset \ldots \subset L_{n}^{\prime}\right)$ of vector subbundles on $L_{n}^{\prime}$ with isomorphisms

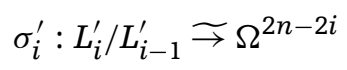


for $1 \leq i \leq n$. We have a morphism $\tilde{\varphi}^{\prime}: \widetilde{\mathcal{Q}}_{n, d}^{\prime} \rightarrow \widetilde{\mathcal{Y}}_{n, d}^{\prime}$ sending the above point to $\left(L^{\prime},\left(s_{i}^{\prime}\right)\right)$, where

$$
s_{i}^{\prime}: \Omega^{(2 n-2)+\ldots+(2 n-2 i)} \underset{\rightarrow}{\sim} \wedge^{i} L_{i}^{\prime} \hookrightarrow \wedge^{i} L^{\prime}
$$

for $1 \leq i \leq n$.

Denote by

$$
\eta: \widetilde{\mathcal{Q}}_{n, d} \times \widetilde{\mathcal{Q}}_{n, d^{\prime}}^{\prime} \rightarrow \mathcal{Q}_{2 n, d+d^{\prime}}
$$

the map sending $\left(L,\left(L_{i}\right), \sigma_{i}\right),\left(L^{\prime},\left(L_{i}^{\prime}\right), \sigma_{i}^{\prime}\right)$ to $\left(M,\left(M_{i}\right),\left(s_{i}\right)\right)$, where $M=L \oplus L^{\prime}, 0=M_{0} \subset M_{1} \subset$ $\ldots \subset M_{2 n}$ is a complete flag of vector bundles on $X$ given by

$$
\begin{cases}M_{2 k+1}=L_{k+1} \oplus L_{k^{\prime}}^{\prime} & 0 \leq k<n \\ M_{2 k}=L_{k} \oplus L_{k^{\prime}}^{\prime} & 1 \leq k \leq n\end{cases}
$$

with the induced trivializations $s_{i}: M_{i} / M_{i-1} \stackrel{\sim}{\rightarrow} \Omega^{2 n-i}$ for $1 \leq i \leq 2 n$.

\subsection{2}

Recall the map $\varphi: \mathcal{Q}_{n, r} \rightarrow \mathcal{Y}_{n, r}$ from Section 3.1.1. We define the commutative diagram

$$
\begin{array}{ccccc}
\mathcal{Y}_{2 n, d+d^{\prime}} & \stackrel{\iota}{\leftarrow} & \widetilde{\mathcal{Y}}_{n, d} \times \widetilde{\mathcal{Y}}_{n, d^{\prime}}^{\prime} & & \\
\uparrow \varphi & & \uparrow & \searrow \bar{\pi} & \\
\mathcal{Q}_{2 n, d+d^{\prime}} & \stackrel{\iota \mathcal{T}}{\leftarrow} & \mathcal{T}^{d, d^{\prime}} & \stackrel{\bar{\pi}^{d, d^{\prime}}}{\rightarrow} & X^{(d)} \times X^{\left(d^{\prime}\right)} \\
& \nwarrow \eta & \uparrow \bar{\eta} & & \\
& & \widetilde{\mathcal{Q}}_{n, d} \times \widetilde{\mathcal{Q}}_{n, d^{\prime}}^{\prime} & &
\end{array}
$$

as follows. We require the top left square in this diagram to be Cartesian, so defining the stack $\mathcal{T}^{d, d^{\prime}}$ and maps $\iota_{\mathcal{T}}, \bar{\pi} d, d^{\prime}$. We define $\bar{\eta}$ as the morphism whose composition with $\iota_{\mathcal{T}}$ is $\eta$, and whose composition with $\mathcal{T}^{d, d^{\prime}} \rightarrow \tilde{\mathcal{Y}}_{n, d} \times \tilde{\mathcal{Y}}_{n, d^{\prime}}^{\prime}$ is $\tilde{\varphi} \times \tilde{\varphi}^{\prime}$.

Remark 4.2.3. The map $\bar{\eta}$ is a closed immersion. Indeed, let us be given a point of $\mathcal{T}^{d, d^{\prime}}$, which is a collection $\left(L,\left(s_{i}\right)\right) \in \tilde{\mathcal{Y}}_{n, d},\left(L^{\prime},\left(s_{i}^{\prime}\right)\right) \in \tilde{\mathcal{Y}}_{n, d^{\prime}}^{\prime}$ and a flag

$$
\left(M_{1} \subset \ldots \subset M_{2 n} \subset L \oplus L^{\prime}\right)
$$


with trivializations $M_{i} / M_{i-1} \underset{\rightarrow}{\rightarrow} \Omega^{2 n-i}$ for $1 \leq i \leq 2 n$ such that the corresponding maps $\wedge^{i} M_{i} \hookrightarrow \wedge^{i}\left(L \oplus L^{\prime}\right)$ coincide with $t_{i}$ defined by (10).

Assume by induction on $k$, we have already constructed $\left(L_{1} \subset \ldots \subset L_{k} \subset L\right)$ and $\left(L_{1}^{\prime} \subset \ldots \subset L_{k}^{\prime} \subset L^{\prime}\right)$ with $M_{2 i}=L_{i} \oplus L_{i}^{\prime}$ for $0 \leq 2 i \leq 2 k$ and $M_{2 i+1}=L_{i+1} \oplus L_{i}$ for $0 \leq 2 i+1<2 k$. Then, our point of $\mathcal{T}^{d, d^{\prime}}$ gives rise to a section $\Omega^{2 n-2 k-1} \hookrightarrow\left(L / L_{k}\right) \oplus\left(L^{\prime} / L_{k}^{\prime}\right)$. The condition that its component $\Omega^{2 n-2 k-1} \rightarrow L^{\prime} / L_{k}^{\prime}$ vanishes is closed, and gives rise to a subsheaf $L_{k+1} \subset L$. One gets a subsheaf $L_{k+1}^{\prime} \subset L^{\prime}$ similarly and then continues by induction on $k$.

The following is the main result of Section 4.2. Its proof is given in Section 4.2.8.

Proposition 4.2.4. For any local system $E$ on $X$ the natural map

$$
\bar{\pi}_{!}^{d, d^{\prime}} \iota_{\mathcal{T}}^{*}\left(\mathcal{F}_{2 n, E}^{d+d^{\prime}}\right) \rightarrow \bar{\pi}_{!}^{d, d^{\prime}} \bar{\eta}_{*} \bar{\eta}^{*} \iota_{\mathcal{T}}^{*}\left(\mathcal{F}_{2 n, E}^{d+d^{\prime}}\right)
$$

induces an isomorphism on the usual cohomology sheaves in degree $n(g-1)-2 d^{\prime}$.

Remark 4.2.5. By Proposition 4.1.2, the left hand side of (29) is placed in usual cohomological degree $n(g-1)-2 d^{\prime}$ only.

4.2.6

For $\lambda \in \Lambda_{2 n, d+d^{\prime}}^{\succ \text { pos }}$ with $\sum_{i=1}^{n} \lambda_{2 i-1}=d$ and $\sum_{i=1}^{n} \lambda_{2 i}=d^{\prime}$, let $\mathcal{T}^{\lambda}$ denote the preimage of ${ }^{2 n} \mathcal{Z}^{\lambda}$ under the composition

$$
\mathcal{T}^{d, d^{\prime}} \rightarrow \widetilde{\mathcal{Y}}_{n, d} \times \widetilde{\mathcal{Y}}_{n, d^{\prime}}^{\prime} \stackrel{i \mathcal{Y}}{\rightarrow} 2 n_{\mathcal{Z}^{d, d^{\prime}}}
$$

Let $\bar{\eta}^{\lambda}: \widetilde{\mathcal{Q}} \widetilde{\mathcal{Q}}^{\prime \lambda} \hookrightarrow \mathcal{T}^{\lambda}$ be obtained from $\bar{\eta}$ by the base change $\mathcal{T}^{\lambda} \rightarrow \mathcal{T}^{d, d^{\prime}}$. Let $\bar{\pi}^{\lambda}: \mathcal{T}^{\lambda} \rightarrow$ $X_{\text {pos }}^{\lambda}$ be the composition $\mathcal{T}^{\lambda} \rightarrow{ }^{2 n} \mathcal{Z}^{\lambda} \rightarrow X_{\text {pos }}^{\lambda}$. Clearly, $\bar{\pi}^{\lambda}$ factors through the closed subscheme $X^{\lambda} \hookrightarrow X_{\text {pos }}^{\lambda}$.

Write $\iota_{\mathcal{T}}^{\lambda}: \mathcal{T}^{\lambda} \rightarrow \mathcal{Q}_{2 n, d+d^{\prime}}$ for the restriction of $\iota_{\mathcal{T}}$. We get the diagram

$$
\begin{array}{ccc}
\mathcal{Q}_{2 n, d+d^{\prime}} \stackrel{\iota^{\lambda}}{\stackrel{\mathcal{T}}{\leftarrow}} & \mathcal{T}^{\lambda} \stackrel{\bar{\pi}^{\lambda}}{\rightarrow} X^{\lambda} \\
& \uparrow^{\uparrow} \bar{\eta}^{\lambda} \\
& \widetilde{\mathcal{Q}} \widetilde{\mathcal{Q}}^{\prime \lambda}
\end{array}
$$


Denote by

$$
\varphi^{\lambda}: \mathcal{T}^{\lambda} \rightarrow{ }^{2 n} \mathcal{Z}^{\lambda} \times_{X_{p o s}^{\lambda}} X^{\lambda}
$$

the map obtained from $\varphi: \mathcal{Q}_{2 n, d+d^{\prime}} \rightarrow \mathcal{Y}_{2 n, d+d^{\prime}}$ by the base change ${ }^{2 n} \mathcal{Z}^{\lambda} \times_{X_{\text {pos }}^{\lambda}} X^{\lambda} \rightarrow$ $2 n \mathcal{Z}^{\lambda} \rightarrow \mathcal{Y}_{2 n, d+d^{\prime}}$. The map $\varphi^{\lambda}$ is a generalized affine fibration.

We derive Proposition 4.2.4 from to the following result, whose proof is given in Section 4.2.9.

Proposition 4.2.7. For $\lambda \in \Lambda_{2 n, d+d^{\prime}}^{\succ}$ the natural map

$$
\varphi_{!}^{\lambda}\left(\iota_{\mathcal{T}}^{\lambda}\right)^{*}\left(\mathcal{F}_{2 n, E}^{d+d^{\prime}}\right) \rightarrow \varphi_{!}^{\lambda}\left(\bar{\eta}^{\lambda}\right)_{!}\left(\bar{\eta}^{\lambda}\right)^{*}\left(\iota_{\mathcal{T}}^{\lambda}\right)^{*}\left(\mathcal{F}_{2 n, E}^{d+d^{\prime}}\right)
$$

induces an isomorphism on the (highest) cohomology sheaves in the usual degree

$$
2 a_{2 n}(\lambda)-b\left(2 n, d+d^{\prime}\right)
$$

Both complexes are placed in the usual cohomological degrees $\leq 2 a_{2 n}(\lambda)-b\left(2 n, d+d^{\prime}\right)$.

\subsubsection{Proof of Proposition 4.2.4}

It suffices to show that for any $\lambda \in \Lambda_{2 n, d+d^{\prime}}^{\succ}$ with $\sum_{i=1}^{n} \lambda_{2 i-1}=d$ and $\sum_{i=1}^{n} \lambda_{2 i}=d^{\prime}$ the natural map

$$
\bar{\pi}_{!}^{\lambda}\left(\iota_{\mathcal{T}}^{\lambda}\right)^{*}\left(\mathcal{F}_{2 n, E}^{d+d^{\prime}}\right) \rightarrow \bar{\pi}_{!}^{\lambda} \bar{\eta}_{*}^{\lambda}\left(\bar{\eta}^{\lambda}\right)^{*}\left(\iota_{\mathcal{T}}^{\lambda}\right)^{*}\left(\mathcal{F}_{2 n, E}^{d+d^{\prime}}\right)
$$

induces an isomorphism on the usual cohomology sheaves in degree $n(g-1)-2 d^{\prime}$. Since $q_{\lambda}:{ }^{2 n} \mathcal{Z}_{-}^{\lambda} \rightarrow X_{-}^{\lambda}$ is a generalized affine fibration of rank (27), our claim follows from Proposition 4.2.7.

\subsubsection{Proof of Proposition 4.2.7}

For $\lambda \in \Lambda_{2 n, d+d^{\prime}}^{\succ}$ the stack $\mathcal{T}^{\lambda}$ classifies collections:

- complete flags $\left(L_{1} \subset \ldots \subset L_{n}=L\right)$ and $\left(L_{1}^{\prime} \subset \ldots \subset L_{n}^{\prime}=L^{\prime}\right)$ of vector bundles on $X$, for which we set

$$
\begin{array}{ll}
M_{2 i}=L_{i} \oplus L_{i}^{\prime}, & \text { for } 0 \leq 2 i \leq 2 n \\
M_{2 i+1}=L_{i+1} \oplus L_{i}^{\prime}, & \text { for } 0<2 i+1<2 n
\end{array}
$$


- $\left(D_{i}\right) \in X^{\lambda}$;

- isomorphisms $M_{i} / M_{i-1} \stackrel{\sim}{\rightarrow} \Omega^{2 n-i}\left(D_{i}\right)$ for $1 \leq i \leq 2 n$;

- lower modifications of $\mathcal{O}_{X}$-modules $\mathcal{M}_{i} \subset M_{i}$ for $1 \leq i \leq 2 n$ such that

$$
\mathcal{M}_{1} \subset \mathcal{M}_{2} \subset \ldots \subset \mathcal{M}_{2 n}
$$

is a complete flag of vector bundles on $X$, and the image of $\mathcal{M}_{i} / \mathcal{M}_{i-1} \rightarrow$ $M_{i} / M_{i-1}$ is $\left(M_{i} / M_{i-1}\right)\left(-D_{i}\right)$ for $1 \leq i \leq 2 n$.

Pick a $k$-point $\xi$ of ${ }^{2 n} \mathcal{Z}^{\lambda} \times_{X_{\text {pos }}^{\lambda}} X^{\lambda}$ given by a collection: $\left(D_{i}\right) \in X^{\lambda}$, complete flags of vector bundles on $X$

$$
\left(L_{1} \subset \ldots \subset L_{n}=L, \quad L_{1}^{\prime} \subset \ldots \subset L_{n}^{\prime}=L^{\prime}\right),
$$

and isomorphisms (18) and (19) for $1 \leq i \leq n$. It suffices to establish the desired result after the $*$-restriction to $\xi$. Write $S$ for the fibre of $\varphi^{\lambda}$ over $\xi$. Define $M_{i}$ by (30).

For $x \in X$, write $\mathcal{O}_{X}$ for the completed local ring of $X$ at $X, F_{X}$ for the fraction field of $\mathcal{O}_{X}$, set $D_{X}=\operatorname{Spec} \mathcal{O}_{X}$. Let

$$
\left(L_{1, X} \subset \ldots \subset L_{n, X}=L_{X^{\prime}} \quad L_{1, X}^{\prime} \subset \ldots \subset L_{n, X}^{\prime}=L_{X}^{\prime}\right)
$$

be the restriction of (31) to $D_{X^{\prime}}$, similarly for $M_{i_{X}}$. Write

$$
D_{i}=\sum_{x \in X} \lambda_{i, X} X
$$

so for $x \in X$ we get the $x$-component $\lambda_{X} \in \Lambda_{2 n}^{\succ}$ of $D=\left(D_{i}\right)$, and $D=\sum_{X \in X} \lambda_{X} X$.

For $x \in X$ let $S_{X}$ be the scheme classifying diagrams

$$
\begin{array}{ccc}
M_{1, X} & \subset \ldots \subset & M_{2 n, X} \\
\cup & & \cup \\
\mathcal{M}_{1, X} & \subset \ldots \subset & \mathcal{M}_{2 n, X^{\prime}}
\end{array}
$$

where $\mathcal{M}_{i, X} \subset M_{i, X}$ is a $\mathcal{O}_{X}$-submodule such that for $1 \leq i \leq 2 n$ the natural map

$$
\mathcal{M}_{i, X} / \mathcal{M}_{i-1, X} \rightarrow M_{i, X} / M_{i-1, X}
$$

is an inclusion with image $\left(M_{i, X} / M_{i-1, X}\right)\left(-\lambda_{i, X} X\right)$. Note that $S_{X}$ is isomorphic to an affine space of dimension $a_{2 n}\left(\lambda_{X}\right)$. We have canonically $S \widetilde{\rightarrow} \prod_{X \in X} S_{X}$. 
For $x \in X$ define the map $\mu_{X}: S_{X} \rightarrow \mathbb{A}^{1}$ as follows. For $1 \leq i<2 n$ we have

$$
M_{i+1} / M_{i-1} \stackrel{\widetilde{\rightarrow}}{\rightarrow} M_{i} / M_{i-1} \oplus M_{i+1} / M_{i}
$$

canonically. So, for $1 \leq i<2 n$, a point of $S_{X}$ yields an inclusion

$$
\begin{aligned}
& \Omega_{X}^{2 n-i-1} \underset{\rightarrow}{\rightarrow} \mathcal{M}_{i+1, X} / \mathcal{M}_{i, X} \subset M_{i+1, X} /\left(\mathcal{M}_{i, X}+M_{i-1, X}\right) \stackrel{\sim}{\rightarrow} \\
& \quad\left(\Omega_{X}^{2 n-i}\left(\lambda_{i, X} X\right) / \Omega_{X}^{2 n-i}\right) \oplus \Omega_{X}^{2 n-i-1}\left(\lambda_{i+1, X} X\right)
\end{aligned}
$$

whose projection to the second factor is the canonical inclusion. Let $\sigma_{i, X}$ be the first component of the latter map. Then, $\mu_{X}$ sends a point of $S_{X}$ to $\sum_{i=1}^{2 n-1} \operatorname{Res} \sigma_{i, X}$.

Recall the map $\mu: \mathcal{Q}_{2 n, d+d^{\prime}} \rightarrow \mathbb{A}^{1}$ defined in Section 3.1.1. The composition $S \subset \mathcal{Q}_{2 n, d+d^{\prime}} \stackrel{\mu}{\rightarrow} \mathbb{A}^{1}$ writes as $\sum_{X} \mu_{X}: \prod_{X \in X} S_{X} \rightarrow \mathbb{A}^{1}$. Set

$$
d_{X}=\sum_{i=1}^{n} \lambda_{2 i-1, X}, \quad d_{X}^{\prime}=\sum_{i=1}^{n} \lambda_{2 i, X}
$$

Write $\beta_{X}: S_{X} \rightarrow \operatorname{Sh}_{0}^{d_{X}+d_{x}^{\prime}}$ for the map sending the above point to $M_{2 n, X} / \mathcal{M}_{2 n, X}$.

Write $\operatorname{Gr}\left(M_{2 n, x}\right)$ for the affine Grassmannian classifying free $\mathcal{O}_{X}$-submodules $\mathcal{R} \subset M_{2 n, X}\left(F_{X}\right)$ of rank $2 n$ with $\mathcal{R} \otimes_{\mathcal{O}_{X}} F_{X}=M_{2 n, X}\left(F_{X}\right)$. We view $S_{X}$ as a subscheme of $\operatorname{Gr}\left(M_{2 n, X}\right)$. For $v \in \Lambda_{2 n}^{\succ+}$ let $\operatorname{Gr}^{v}\left(M_{2 n, X}\right) \subset \operatorname{Gr}\left(M_{2 n, X}\right)$ be the locally closed subscheme classifying lattices $\mathcal{R} \subset M_{2 n, x}$ such that

$$
M_{2 n, X} / \mathcal{R} \underset{\rightarrow}{\rightarrow} \mathcal{O}_{X} / \mathfrak{m}_{X}^{\nu_{1}} \oplus \ldots \oplus \mathcal{O}_{X} / \mathfrak{m}_{X}^{\nu_{2 n}}
$$

here $\mathfrak{m} \subset \mathcal{O}_{X}$ is the maximal ideal. The IC-sheaf of $\mathrm{Gr}^{\nu}\left(M_{2 n, X}\right)$ on $\operatorname{Gr}\left(M_{2 n, X}\right)$ is denoted $\mathcal{A}_{v, X}$.

By ([1], Proposition 3.1 and Lemma 4.2), the $*$-restriction of $\left(\iota_{\mathcal{T}}^{\lambda}\right)^{*} \beta^{*} \mathcal{L}_{E}^{d+d^{\prime}}$ to $S$ identifies with $\underset{x \in X}{\bigotimes} F_{X}$, where $F_{X}$ is the $*$-restriction of

$$
\underset{v \in \Lambda_{2 n, d_{X}+d_{X}^{\prime}}^{\succ+}}{\bigoplus} \mathcal{A}_{v, X}\left[-\left(d_{X}+d_{X}^{\prime}\right)(2 n-1)\right] \otimes E_{X}^{v}
$$

under $S_{X} \hookrightarrow \operatorname{Gr}\left(M_{2 n, X}\right)$. Here $E_{X}^{v}$ is the polynomial functor of the vector space $E_{X}$ defined in $([11], 0.1 .4)$. 
Let $i_{M}: S_{M, X} \subset S_{X}$ be the closed subscheme given by the property that there are diagrams of $\mathcal{O}_{X}$-modules

$$
\begin{aligned}
& L_{1, x} \quad \subset \ldots \subset L_{n, X} \quad L_{1, x}^{\prime} \quad \subset \ldots \subset L_{n, X}^{\prime} \\
& \cup \quad \cup \quad \cup \quad \cup \\
& \mathcal{L}_{1, X} \quad \subset \ldots \subset \mathcal{L}_{n, X} \quad \mathcal{L}_{1, X}^{\prime} \quad \subset \ldots \subset \quad \mathcal{L}_{n, X}^{\prime}
\end{aligned}
$$

such that

$$
\begin{cases}\mathcal{M}_{2 s, X}=\mathcal{L}_{s, X} \oplus \mathcal{L}_{s, X^{\prime}}^{\prime} & \text { for } 1 \leq 2 s \leq 2 n \\ \mathcal{M}_{2 s+1, X}=\mathcal{L}_{s+1, X} \oplus \mathcal{L}_{s, X^{\prime}}^{\prime} & \text { for } 1 \leq 2 s+1 \leq 2 n\end{cases}
$$

By Kunneth formula, Proposition 4.2.7 is reduced to Proposition 4.2.10, which is a local result.

Proposition 4.2.10. For $x \in X$ the complex $\mathrm{R} \Gamma_{C}\left(S_{M_{,} X^{\prime}} i_{M}^{*}\left(F_{X} \otimes \mu_{X}^{*} \mathcal{L}_{\psi}\right)\right)$ is placed in degrees $\leq 2 a_{2 n}\left(\lambda_{X}\right)$. Moreover, the natural map

$$
\mathrm{R} \Gamma_{C}\left(S_{X^{\prime}} F_{X} \otimes \mu_{X}^{*} \mathcal{L}_{\psi}\right) \rightarrow \mathrm{R} \Gamma_{C}\left(S_{M, X}, i_{M}^{*}\left(F_{X} \otimes \mu_{X}^{*} \mathcal{L}_{\psi}\right)\right)
$$

induces an isomorphism in the highest degree $2 a_{2 n}\left(\lambda_{X}\right)$.

Proof. Using (33), our claim is reduced to the following. For any $v \in \Lambda_{2 n, d_{x}+d_{x}^{\prime}}^{\succ+}$ the natural map

$$
\mathrm{R} \Gamma_{C}\left(S_{X}, \mathcal{A}_{v_{,} X} \otimes \mu_{X}^{*} \mathcal{L}_{\psi}\right) \rightarrow \mathrm{R} \Gamma_{C}\left(S_{M_{,},} i_{M}^{*}\left(\mathcal{A}_{v_{,} X} \otimes \mu_{X}^{*} \mathcal{L}_{\psi}\right)\right)
$$

is an isomorphism in the (highest) degree $\left\langle\lambda_{x}, 2 \check{\rho}_{G}\right\rangle$ for $G=G_{2 n}$. Besides, both complexes are placed in degrees $\leq\left\langle\lambda_{X}, 2 \check{\rho}_{G}\right\rangle$. This is a particular case of Proposition A.2.2. Namely, we apply it for $G=G_{2 n}$ with $T$ the group of diagonal matrices, $B$ the group of upper triangular matrices. The antistandard Levi is the subgroup of $\mathrm{GL}_{2 n}$ preserving the decomposition $\operatorname{Vect}\left(e_{1}, e_{3}, \ldots, e_{2 n-1}\right) \oplus \operatorname{Vect}\left(e_{2}, e_{4}, \ldots, e_{2 n}\right)$, where $\left(e_{i}\right)$ is the canonical base of the standard representation of $\mathrm{GL}_{2 n}$.

The proof of Proposition 4.2.4 is completed. 


\subsection{Highest direct image: second step}

In this section, we finish the proof of Proposition 2.3.5. More precisely, we reduce it to Proposition 4.3.2, which in turn is reduced to Theorem 4.3.9. This reduction is the content of Section 4.3. The proof of Theorem 4.3.9 is then given in Section 4.4.

\subsection{1}

Write $\tilde{\beta}: \widetilde{\mathcal{Q}}_{n, d} \rightarrow{ }^{\leq n} \mathrm{Sh}_{0}^{d}$ for the map sending a point of the source to $L / L_{n}$. Write $\tilde{\beta}^{\prime}$ : $\widetilde{\mathcal{Q}}_{n, d^{\prime}}^{\prime} \rightarrow{ }^{\leq n} \mathrm{Sh}_{0}^{d^{\prime}}$ for the map sending a point of the source to $L^{\prime} / L_{n}^{\prime}$.

Consider the commutative diagram

$$
\begin{array}{ccccc}
\mathcal{Q}_{2 n, d+d^{\prime}} & \stackrel{\eta}{\leftarrow} & \widetilde{\mathcal{Q}}_{n, d} \times \widetilde{\mathcal{Q}}_{n, d^{\prime}}^{\prime} & \stackrel{\bar{\pi}^{d, d^{\prime}} \bar{\eta}}{\rightarrow} X^{(d)} \times X^{\left(d^{\prime}\right)} \\
\downarrow \beta & & \downarrow \tilde{\beta} \times \tilde{\beta}^{\prime} & \nearrow \leq n(\operatorname{div} \times \operatorname{div}) \\
\leq 2 n \mathrm{Sh}_{0}^{d+d^{\prime}} & \stackrel{\eta_{0}}{\leftarrow} \quad \leq n \mathrm{Sh}_{0}^{d} \times \leq n \operatorname{Sh}_{0}^{d^{\prime},} &
\end{array}
$$

where $\eta_{0}$ sends $\left(F, F^{\prime}\right)$ to $F \oplus F^{\prime}$, and $\leq(\operatorname{div} \times \operatorname{div})$ is the restriction of $\operatorname{div} \times \operatorname{div}$.

For any local system $E$ on $X$, we define a canonical constructible subsheaf

$$
{ }^{\leq n}\left(\left(\wedge^{2} E\right)^{(d)} \otimes E^{\left(d^{\prime}-d\right)}\right) \subset\left(\left(\wedge^{2} E\right)^{(d)} \otimes E^{\left(d^{\prime}-d\right)}\right)
$$

in Definition 4.3.11. We will derive Proposition 2.3.5 from the following Proposition 4.3.2, whose proof is given in Section 4.3.12.

Proposition 4.3.2. For any local system $E$ on $X$, the complex

$$
i_{X}^{*}(\leq n(\operatorname{div} \times \operatorname{div}))_{!}\left(\tilde{\beta} \times \tilde{\beta}^{\prime}\right)_{!}\left(\tilde{\beta} \times \tilde{\beta}^{\prime}\right)^{*} \eta_{0}^{*} \mathcal{L}_{E}^{d+d^{\prime}}
$$

is placed in the usual cohomological degrees $\leq b\left(2 n, d+d^{\prime}\right)+n(g-1)-2 d^{\prime}=t o p$, and its cohomology sheaf in degree top identifies canonically with

$$
\leq n\left(\left(\wedge^{2} E\right)^{(d)} \otimes E^{\left(d^{\prime}-d\right)}\right)
$$

If $\operatorname{rk}(E)=2 n$ then (12) is an equality. 


\subsubsection{Proof of Proposition 2.3.5}

Consider the diagram (28). One has canonically over $\tilde{\mathcal{Y}}_{n, d} \times \widetilde{\mathcal{Y}}_{n, d^{\prime}}^{\prime}$

$$
\iota^{*}\left(\mathcal{P}_{2 n, E}^{d+d^{\prime}}\right)\left[n(g-1)-d^{\prime}\right] \underset{\rightarrow}{\sim} q_{\mathcal{Y}}^{*}\left(\mathcal{P}_{2 n, E} \otimes \overline{\mathbb{Q}}_{\ell}\right)\left[\operatorname{dim} . \operatorname{rel}\left(v_{\mathcal{Z}}\right)\right]
$$

So, in view of Proposition 4.1.2, to prove Proposition 2.3.5, we must calculate the cohomology sheaf in the usual degree $n(g-1)-2 d^{\prime}$ of the complex

$$
\bar{\pi}_{!}^{d, d^{\prime}} \iota_{\mathcal{T}}^{*}\left(\mathcal{F}_{2 n, E}^{d+d^{\prime}}\right)
$$

By Proposition 4.2.4, this cohomology sheaf identifies with the cohomology sheaf in the same degree of

$$
\bar{\pi}_{!}^{d, d^{\prime}} \bar{\eta}_{!} \eta^{*}\left(\mathcal{F}_{2 n, E}^{d+d^{\prime}}\right)
$$

From (35) one gets

$$
\eta^{*}\left(\mathcal{F}_{2 n, E}^{d+d^{\prime}}\right) \underset{\rightarrow}{\rightarrow} \eta^{*} \beta^{*} \mathcal{L}_{E}^{d+d^{\prime}}\left[b\left(2 n, d+d^{\prime}\right)\right]
$$

Our claim follows now from Proposition 4.3.2.

Remark 4.3.4. Recall the sheaf $E_{-}^{\lambda}$ defined in ([11], Definition 1, Section 3.1). In the case $n=1$, the top cohomology sheaf of (12) identifies with $E_{-}^{\lambda}$ on $X_{-}^{\lambda}=X^{(d)} \times X^{\left(d^{\prime}-d\right)}$ for $\lambda=\left(d, d^{\prime}\right)$ by definition of $E_{-}^{\lambda}$.

\subsection{5}

In the rest of Section 4.3, we reduce Proposition 4.3.2 to Theorem 4.3.9.

For the rest of Section 4 , assume $0 \leq d \leq d^{\prime}$ and set $I=\left\{1, \ldots, d+d^{\prime}\right\}$. Let $S_{I}$ be the group of permutations of $I$.

Set

$$
V^{d, d^{\prime}}=\left(X^{(d)} \times X^{\left(d^{\prime}\right)}\right) \times_{X^{\left(d+d^{\prime}\right)}} X^{d+d^{\prime}}
$$

This is the scheme classifying pairs of effective divisors $D, D^{\prime}$ of degrees $d, d^{\prime}$, and $\left(x_{i}\right) \in$ $X^{d+d^{\prime}}$ with $\sum_{i} x_{i}=D+D^{\prime}$. Write $V_{-}^{d, d^{\prime}} \hookrightarrow V^{d, d^{\prime}}$ for the closed subscheme given by $D \leq D^{\prime}$. It is equipped with an action of $S_{I}$. 


\subsubsection{Description of the IC-sheaf}

Consider the set $\mathcal{E}$ of equivalence classes of surjections $\alpha: I \rightarrow \bar{I}$ such that there is a decomposition $\bar{I}=\bar{I}_{1} \sqcup \bar{I}_{2}$ with $\left|\bar{I}_{2}\right|=d^{\prime}-d$ and the following property. For $i \in \bar{I}_{2}$ (resp., $i \in \bar{I}_{1}$ ), $\alpha^{-1}(i)$ has one (resp., 2) elements. In other words, $\mathcal{E}$ is the set of equivalence relations on $I$, which have $d^{\prime}-d$ equivalence classes consisting of one element and $d$ equivalence classes consisting of two elements. We get a map

$$
\text { norm }: \underset{\alpha \in \mathcal{E}}{\sqcup} X^{\bar{I}} \rightarrow V_{-}^{d, d^{\prime}}
$$

whose restriction to $X^{\bar{I}}$ sends $\gamma: \bar{I} \rightarrow X$ to $D=\sum_{i \in \bar{I}_{1}} \gamma(i), D^{\prime}=D+\sum_{i \in \bar{I}_{2}} \gamma(i)$ and the decomposition $I \rightarrow X$ of $D+D^{\prime}$ is given by $\gamma \alpha$. This is the normalization of $V_{-}^{d, d^{\prime}}$. So, norm $\overline{\mathbb{Q}}_{\ell}\left[d^{\prime}\right] \stackrel{\sim}{\rightarrow}$ IC, and $V_{-}^{d, d^{\prime}}$ is of pure dimension $d^{\prime}$. The map norm is $S_{I^{-}}$equivariant.

Denote by ${ }^{0} V_{-}^{d, d^{\prime}} \subset V_{-}^{d, d^{\prime}}$ the open subscheme given by the property that $D$ is reduced, and $\left(D^{\prime}-D\right) \cap D=\emptyset$. Then norm is an isomorphism over this open subscheme, so IC $\underset{\rightarrow}{\widetilde{Q}} \overline{\mathbb{Q}}_{\ell}[\mathrm{dim}]$ over ${ }^{0} V_{-}^{d, d^{\prime}}$.

For each $(\bar{I}, \alpha) \in \mathcal{E}$, the restriction norm $_{\alpha}: X^{\bar{I}} \rightarrow V_{-}^{d, d^{\prime}}$ of norm is a closed immersion. Indeed, the resulting closed subscheme is given by the property that a point $I \rightarrow X$ factors (uniquely) through $I \stackrel{\alpha}{\rightarrow} \bar{I} \stackrel{\gamma}{\rightarrow} X$, and $D=\sum_{i \in \bar{I}_{1}} \gamma(i)$. We denote by $V_{\alpha}$ this closed subscheme of $V_{-}^{d, d^{\prime}}$.

By an involution of $I$ we mean a permutation $\sigma$ with $\sigma^{2}=$ id, so $\sigma$ could be the identity. Write Inv for the set of involutions in $S_{I}$. For $\alpha: I \rightarrow \bar{I}$ in $\mathcal{E}$, let $w \in \operatorname{Inv}$ be the unique involution of $I$ whose orbits are precisely the fibres of $\alpha$. This defines an inclusion $\mathcal{E} \hookrightarrow \operatorname{Inv}$, which we use later.

For $\alpha \in \mathcal{E}$ denote by $S_{\alpha}$, the stabilizer of $\alpha$ in $S_{I}$. Set $I_{i}=\alpha^{-1}\left(\bar{I}_{i}\right)$ for $i=1,2$. Write $S_{I_{i}}$ for the group of permutations of $I_{i}$, so $S_{\alpha} \subset S_{I_{1}} \times S_{I_{2}} \subset S_{I}$. Let $\chi_{\alpha}: S_{\alpha} \rightarrow \overline{\mathbb{Q}}_{\ell}^{*}$ be the restriction of the character sign $\times$ triv $: S_{I_{1}} \times S_{I_{2}} \rightarrow \overline{\mathbb{Q}}_{\ell}^{*}$. The group $S_{\alpha}$ fits into an exact sequence $1 \rightarrow\left(S_{2}\right)^{d} \rightarrow S_{\alpha} \rightarrow S_{\bar{I}_{1}} \times S_{\bar{I}_{2}} \rightarrow 1$.

\subsubsection{Twisted IC-sheaf}

We introduce the following canonical induced representation of $S_{I}$. The term canonical here is to express the fact that it is independent of the order on $I$ (and only depends on $\left.I, d, d^{\prime}\right)$.

Let $\left\{e_{i}\right\}_{i \in I}$ be the canonical base of $\overline{\mathbb{Q}}_{\ell}^{I}$. For $\alpha: I \rightarrow \bar{I}$ in $\mathcal{E}$ and $j \in \bar{I}_{1}$ set

$$
V_{j, \alpha}=\bigoplus_{i \in \alpha^{-1}(j)} \overline{\mathbb{Q}}_{\ell} e_{i} \subset \overline{\mathbb{Q}}_{\ell}^{I}
$$


Let

$$
V_{\alpha}=\otimes_{j \in \bar{I}_{1}} \operatorname{det} V_{j, \alpha}, \quad \operatorname{Ind}_{\mathcal{E}}=\underset{\alpha \in \mathcal{E}}{\bigoplus} V_{\alpha}
$$

It is understood that $\bar{I}_{1}$ is attached to an element $\alpha: I \rightarrow \bar{I}$ of $\mathcal{E}$ as above. Equip Ind $_{\mathcal{E}}$ with the natural action of $S_{I}$. One may view $\operatorname{In} d_{\mathcal{E}}$ as a subspace in $\left(\overline{\mathbb{Q}}_{\ell}^{I}\right)^{\otimes 2 d}$. For $\alpha \in \mathcal{E}$, there exists a noncanonical isomorphism $\operatorname{Ind} d_{\mathcal{E}} \underset{\rightarrow}{\rightarrow} i n d_{S_{\alpha}}^{S_{I}} \chi_{\alpha}$ of $S_{I}$-representations. We underline that $I n d_{\mathcal{E}}$ is independent of $\alpha \in \mathcal{E}$.

Consider the sheaf $\left(\overline{\mathbb{Q}}_{\ell}\right)_{\mathcal{E}}$ on $\underset{\alpha \in \mathcal{E}}{\sqcup} X^{\bar{I}}$, whose restriction to the component $X^{\bar{I}}$ for a given $\alpha$ is the constant sheaf with fibre $V_{\alpha}$. Then, $\left(\overline{\mathbb{Q}}_{\ell}\right)_{\mathcal{E}}$ is naturally $S_{I}$-equivariant. Set

$$
\widetilde{\mathrm{IC}}\left[-d^{\prime}\right]=\operatorname{norm}_{!}\left(\overline{\mathbb{Q}}_{\ell}\right)_{\mathcal{E}}
$$

This is a constructible sheaf on $V_{-}^{d, d^{\prime}}$, which inherits a natural action of $S_{I}$.

We call $\widetilde{\mathrm{IC}}$ the twisted version of the IC-sheaf of $V_{-}^{d, d^{\prime}}$. We informally think of $\widetilde{\mathrm{IC}}$ as a version of the canonical induced representation of $S_{I}$ with a point replaced by the curve $X$. There is a noncanonical isomorphism $\widetilde{\mathrm{IC}} \underset{\rightarrow}{\Im} \mathrm{IC}$.

\section{3 .8}

For the rest of Section 4 , set $v=(1, \ldots, 1) \in \Lambda_{d+d^{\prime}, d+d^{\prime}}$. Let $\mathcal{F} l^{v}$ be the stack of flags $\left(F_{1} \subset \ldots \subset F_{d+d^{\prime}}\right)$, where $F_{i}$ is a coherent torsion sheaf on $X$ of length $i$ for $i=1, \ldots, d+d^{\prime}$. Let $q_{\mathcal{F}}: \mathcal{F} l^{\nu} \rightarrow \mathrm{Sh}_{0}^{d+d^{\prime}}$ be the map sending the above point to $F_{d+d^{\prime}}$.

Consider the diagram

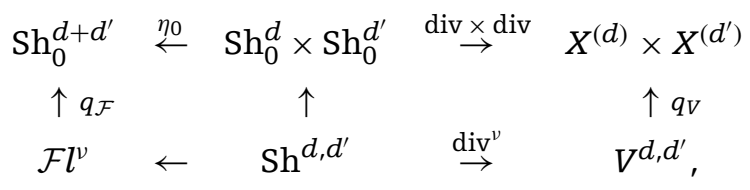

where the left square is Cartesian, so defining the stack $\mathrm{Sh}^{d, d^{\prime}}$. The map $\operatorname{div}^{v}$ sends $\left(F, F^{\prime}, F_{1} \subset \ldots \subset F_{d+d^{\prime}}=F \oplus F^{\prime}\right)$ to the collection $\left(D, D^{\prime},\left(x_{i}\right)\right)$, where $D=\operatorname{div} F, D^{\prime}=\operatorname{div} F^{\prime}$, and $x_{i}=\operatorname{div}\left(F_{i} / F_{i-1}\right)$ for $1 \leq i \leq d+d^{\prime}$. The map $q_{V}$ sends $\left(D, D^{\prime},\left(x_{i}\right)\right)$ to $\left(D, D^{\prime}\right)$. 
Let $\operatorname{div}_{-}^{v}: \mathrm{Sh}_{-}^{d, d^{\prime}} \rightarrow V_{-}^{d, d^{\prime}}$ be obtained from $\operatorname{div}^{v}$ by the base change $V_{-}^{d, d^{\prime}} \hookrightarrow V^{d, d^{\prime}}$. Let $\leq 2 n \mathcal{F} l^{\nu}$ be the preimage of $\leq 2 n \operatorname{Sh}_{0}^{d+d^{\prime}}$ under $q_{\mathcal{F}}: \mathcal{F} l^{\nu} \rightarrow \operatorname{Sh}_{0}^{d+d^{\prime}}$. Consider the diagram

$$
\begin{aligned}
& \leq 2 n \operatorname{Sh}_{0}^{d+d^{\prime}} \quad \stackrel{\eta_{0}}{\leftarrow} \quad \leq n \operatorname{Sh}_{0}^{d} \times \leq n \operatorname{Sh}_{0}^{d^{\prime}} \stackrel{\tilde{\beta} \times \tilde{\beta}^{\prime}}{\leftarrow} \quad \widetilde{\mathcal{Q}}_{n, d} \times \widetilde{\mathcal{Q}}_{n, d^{\prime}}^{\prime} \\
& \uparrow q_{\mathcal{F}} \uparrow \uparrow \\
& \leq 2 n \mathcal{F} l^{\nu} \quad \leftarrow \quad \leq n \operatorname{Sh}^{d, d^{\prime}} \quad \stackrel{\beta^{\nu}}{\leftarrow} \quad \widetilde{\mathcal{Q}} \widetilde{\mathcal{Q}}_{n}^{v},
\end{aligned}
$$

where both squares are Cartesian, so defining the stacks in the low row and $\beta^{\nu}$. Write

$$
\beta_{-}^{v}: \widetilde{\mathcal{Q}} \widetilde{\mathcal{Q}}_{n,-}^{v} \rightarrow{ }^{\leq n} \mathrm{Sh}_{-}^{d, d^{\prime}}
$$

for the map obtained from $\beta^{v}$ by the base change $i_{X}: X^{(d)} \times X^{\left(d^{\prime}-d\right)} \hookrightarrow X^{(d)} \times X^{\left(d^{\prime}\right)}$.

Consider the diagram

$$
\begin{aligned}
& \widetilde{\mathcal{Q}} \widetilde{\mathcal{Q}}_{n,-}^{v} \stackrel{\beta_{-}^{v}}{\rightarrow} \leq n \mathrm{Sh}_{-}^{d, d^{\prime}} \hookrightarrow \mathrm{Sh}_{-}^{d, d^{\prime}} \stackrel{\operatorname{div}_{-}^{v}}{\rightarrow} \quad V_{-}^{d, d^{\prime}} \quad \stackrel{p_{V}}{\rightarrow} X^{d+d^{\prime}} \\
& \downarrow q_{V}^{-} \\
& X^{(d)} \times X^{\left(d^{\prime}-d\right)},
\end{aligned}
$$

where $p_{V}$ sends $\left(D, D^{\prime},\left(x_{i}\right)\right)$ to $\left(x_{i}\right)$, and $q_{V}^{-}$is obtained from $q_{V}$ by the base change $i_{X}$. Let

$$
\leq n \operatorname{div}_{-}^{v}: \leq n \operatorname{Sh}_{-}^{d, d^{\prime}} \rightarrow V_{-}^{d, d^{\prime}}
$$

be the restriction of $\operatorname{div}_{-}^{v}$ to this open substack. The group $S_{I}$ acts naturally on $V_{-}^{d, d^{\prime}}$, $X^{d+d^{\prime}}$, and the map $p_{V}$ is $S_{I^{-}}$equivariant.

Proposition 4.3.2 will be derived from the following results, whose proof is given in Sections 4.3.6-4.4.22.

Theorem 4.3.9. Recall our assumption $0 \leq d \leq d^{\prime}$. i) The map $\operatorname{div}_{-}^{v}: \mathrm{Sh}_{-}^{d, d^{\prime}} \rightarrow V_{-}^{d, d^{\prime}}$ is surjective. Any of its fibres is of dimension $-d^{\prime}$. ii) One has canonically

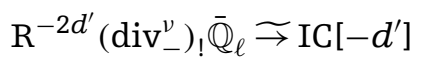

So, for any local system $E$ on $X$,

$$
i_{X}^{*}(\operatorname{div} \times \operatorname{div}) ! \eta_{0}^{*} \mathcal{S} p r_{E}^{d+d^{\prime}}
$$


is placed in usual degrees $\leq-2 d^{\prime}$, and its cohomology sheaf in degree $-2 d^{\prime}$ is isomorphic to

$$
\left(q_{V}^{-}\right)_{!}\left(\widetilde{\mathrm{IC}}\left[-d^{\prime}\right] \otimes p_{V}^{*} E^{\bigotimes\left(d+d^{\prime}\right)}\right)
$$

Moreover, this isomorphism is $S_{I}$-equivariant.

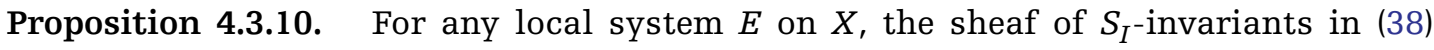
identifies with $\left(\wedge^{2} E\right)^{(d)} \otimes E^{\left(d^{\prime}-d\right)}$.

Proof. Write sum : $X^{d} \rightarrow X^{(d)}$ to the map sending $\left(x_{i}\right)$ to $\sum_{i} x_{i}$. For any $\alpha: I \rightarrow \bar{I}$ in $\mathcal{E}$, the composition

$$
X^{\bar{I}} \stackrel{\text { norm }_{\alpha}}{\rightarrow} V_{-}^{d, d^{\prime}} \stackrel{q_{V}^{-}}{\rightarrow} X^{(d)} \times X^{\left(d^{\prime}-d\right)}
$$

is the map sum $\times$ sum : $X^{d} \times X^{d^{\prime}-d} \rightarrow X^{(d)} \times X^{\left(d^{\prime}-d\right)}$. So,

$$
\left(q_{V}^{-}\right)_{!}\left(\widetilde{\mathrm{IC}}\left[-d^{\prime}\right] \otimes p_{V}^{*} E^{\bigotimes\left(d+d^{\prime}\right)}\right) \underset{\sim}{\rightarrow} \underset{\alpha \in \mathcal{E}}{\oplus}\left(\left(\operatorname{sum}_{!}(E \otimes E)^{\bigotimes d}\right) \otimes\left(\operatorname{sum}_{!} E^{\bigotimes\left(d^{\prime}-d\right)}\right)\right) \otimes V_{\alpha}
$$

according to Section 4.3.6. The group $S_{d+d^{\prime}}$ acts transitively on $\mathcal{E}$, and a stabilizer $S_{\alpha}$ of some $\alpha \in \mathcal{E}$ was considered in Section 4.3.6. It fits into an exact sequence of groups $1 \rightarrow\left(S_{2}\right)^{d} \rightarrow S_{\alpha} \rightarrow\left(S_{d} \times S_{d^{\prime}-d}\right) \rightarrow 1$. So, we are calculating $S_{\alpha}$-invariants on

$$
\left(\left(\operatorname{sum}_{!}(E \otimes E)^{\bigotimes d}\right) \otimes\left(\operatorname{sum}_{!} E^{\bigotimes\left(d^{\prime}-d\right)}\right)\right) \otimes V_{\alpha}
$$

Taking the $\left(S_{2}\right)^{d}$-invariants first, one gets

$$
\left(\operatorname{sum}_{!}\left(\wedge^{2} E\right)^{\bigotimes d}\right) \otimes\left(\operatorname{sum}_{!} E^{\bigotimes\left(d^{\prime}-d\right)}\right),
$$

and this sheaf is equipped with the "induced" action of $S_{d} \times S_{d^{\prime}-d}$. Let $S_{d}$ act naturally on $\operatorname{sum}_{!}\left(\left(\wedge^{2} E\right)^{\bigotimes d}\right)$, and $S_{d^{\prime}-d}$ act naturally on $\operatorname{sum}_{!}\left(E^{\bigotimes\left(d^{\prime}-d\right)}\right)$. Then, the product of these two actions coincides with the 'induced' action of $S_{d} \times S_{d^{\prime}-d}$ on (39). Our claim follows.

Definition 4.3.11. Recall our assumption $0 \leq d \leq d^{\prime}$. Let ${ }^{\leq n} K_{V}$ on $V_{-}^{d, d^{\prime}}$ be given by

$$
{ }^{\leq n} K_{V}=\mathrm{R}^{-2 d^{\prime}}\left({ }^{\leq n} \operatorname{div}_{-}^{v}\right)_{!} \overline{\mathbb{Q}}_{\ell}
$$


Since ${ }^{\leq n} \mathrm{Sh}_{-}^{d, d^{\prime}}$ is open in $\mathrm{Sh}_{-}^{d, d^{\prime}}$, this is a constructible subsheaf of IC[- $\left.d^{\prime}\right]$ on $V_{-}^{d, d^{\prime}}$. We get a $S_{I}$-equivariant filtration by constructible subsheaves

$$
{ }^{\leq 1} K_{V} \subset{ }^{\leq 2} K_{V} \subset \ldots \subset \mathrm{IC}\left[-d^{\prime}\right]
$$

For any local system $E$ on $X$ define the constructible sheaf

$$
\leq n\left(\left(\wedge^{2} E\right)^{(d)} \otimes E^{\left(d^{\prime}-d\right)}\right)
$$

on $X^{(d)} \times X^{\left(d^{\prime}-d\right)}$ as the sheaf of $S_{I^{-}}$invariants in

$$
\left(q_{V}^{-}\right)_{!}\left(p_{V}^{*} E^{\otimes\left(d+d^{\prime}\right)} \otimes{ }^{\leq n} K_{V}\right)
$$

where the $S_{I}$-action comes from the corresponding action on the Springer sheaf.

Since $\left(q_{V}^{-}\right)_{!}$is exact for the usual t-structures, from Proposition 4.3.10 we see that (37) is a constructible subsheaf in $\left(\wedge^{2} E\right)^{(d)} \otimes E^{\left(d^{\prime}-d\right)}$.

\subsubsection{Proof of Proposition 4.3.2}

Recall the maps $\tilde{\beta}: \widetilde{\mathcal{Q}}_{n, d} \rightarrow{ }^{\leq n} \operatorname{Sh}_{0}^{d}$ and $\tilde{\beta}^{\prime}: \widetilde{\mathcal{Q}}_{n, d^{\prime}}^{\prime} \rightarrow{ }^{\leq n} \operatorname{Sh}_{0}^{d^{\prime}}$ from (35). The map $\tilde{\beta}$ is surjective and smooth of relative dimension

$$
n d-\frac{n}{6}(n-1)(1+4 n)(g-1)
$$

The map $\tilde{\beta}^{\prime}$ is surjective and smooth of relative dimension $n d^{\prime}-\frac{n}{6}(n-1)(1+4 n)(g-1)$. All the fibres of $\tilde{\beta}$ and of $\tilde{\beta}^{\prime}$ are irreducible. Since

$$
\operatorname{dim}\left(\mathcal{Q}_{2 n, d+d^{\prime}}\right)=b\left(2 n, d+d^{\prime}\right)=2 n\left(d+d^{\prime}\right)+(1-g) \frac{(2 n-1)}{3} n(4 n-1),
$$

one has

$$
2 \operatorname{dim} \cdot \operatorname{rel}\left(\tilde{\beta} \times \tilde{\beta}^{\prime}\right)=b\left(2 n, d+d^{\prime}\right)+n(g-1)
$$

By definition, (36) identifies canonically with the sheaf of $S_{I}$-invariants in

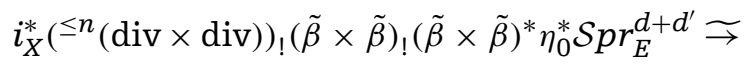

$$
\begin{aligned}
& \left(q_{V}^{-}\right)_{!}\left(p_{V}^{*} E^{\bigotimes\left(d+d^{\prime}\right)} \otimes\left({ }^{\leq n} \operatorname{div}_{-}^{v}\right)_{!}\left(\beta_{-}^{\nu}\right) ! \overline{\mathbb{Q}}_{\ell}\right)
\end{aligned}
$$


Here $S_{I}$ acts via its action on $\mathcal{S p r} r_{E}^{d+d^{\prime}}$. The map $\tilde{\beta} \times \tilde{\beta}^{\prime}$ is surjective and smooth, all its fibres are irreducible. Since $\beta_{-}^{v}$ is obtained by base change from $\tilde{\beta} \times \tilde{\beta}^{\prime},\left(\beta_{-}^{v}\right)$ ! $\overline{\mathbb{Q}}_{\ell}$ is placed in degrees $\leq 2 \operatorname{dim} \operatorname{rel}\left(\tilde{\beta} \times \tilde{\beta}^{\prime}\right)$ and

$$
\mathrm{R}^{2 \operatorname{dim} \cdot \operatorname{rel}\left(\tilde{\beta} \times \tilde{\beta}^{\prime}\right)}\left(\beta_{-}^{v}\right)_{!} \overline{\mathbb{Q}}_{\ell} \underset{\rightarrow}{\rightarrow} \overline{\mathbb{Q}}_{\ell}
$$

By Theorem $4.3 .9 \mathrm{i})$, the complex $\left({ }^{\leq n} \operatorname{div}_{-}^{v}\right)_{!}\left(\beta_{-}^{v}\right) ! \overline{\mathbb{Q}}_{\ell}$ is placed in usual cohomological degrees $\leq 2 \operatorname{dim} . \operatorname{rel}\left(\tilde{\beta} \times \tilde{\beta}^{\prime}\right)-2 d^{\prime}=t o p$, and its top cohomology sheaf is

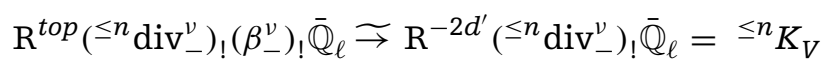

Since $q_{V}^{-}$is finite, (36) is placed in the usual degrees $\leq 2 \operatorname{dim} \cdot \operatorname{rel}\left(\tilde{\beta} \times \tilde{\beta}^{\prime}\right)-2 d^{\prime}$, and the top cohomology sheaf of (36) identifies with (37).

From Proposition 4.1.2, we see that (37) admits a filtration by constructible subsheaves as in Proposition 4.1.2. Assume now $\operatorname{rk}(E)=2 n$. In this case, we check that this filtration exhausts the sheaf

$$
\left(\wedge^{2} E\right)^{(d)} \otimes E^{\left(d^{\prime}-d\right)}
$$

To do so, using Lemma 2.4.2, we check that for any $\left(D, D^{\prime}\right) \in X^{(d)} \times X^{\left(d^{\prime}\right)}$ with $D \leq D^{\prime}$ the *-fibre of $(26)$ at $\left(D, D^{\prime}\right)$ identifies with

$$
\left(\wedge^{2} E\right)_{D}^{(d)} \otimes\left(E^{\left(d^{\prime}-d\right)}\right)_{D^{\prime}-D}
$$

If $D=d x, D^{\prime}=d^{\prime} x$ then this claim is precisely Lemma 2.4.2.

For a $\overline{\mathbb{Q}}_{\ell}$-vector space $V$ and $\lambda \in \Lambda_{2 n}^{\succ-}$ set $V^{\lambda}=V^{w_{0}(\lambda)}$, where $w_{0}$ is the longest element of $S_{d+d^{\prime}}$. In general, we write $D=\sum_{X} d_{X}{ }^{\prime} D^{\prime}=\sum_{X} d_{X}^{\prime} X$, so (41) identifies with

$$
\otimes_{X}\left(\operatorname{Sym}^{d_{x}}\left(\wedge^{2} E_{X}\right) \otimes \operatorname{Sym}^{d_{x}^{\prime}-d_{X}}\left(E_{X}\right)\right)
$$

Set

$$
\mathcal{S}_{X}=\left\{\lambda_{x} \in \Lambda_{2 n, d_{x}+d_{x}^{\prime}}^{\succ-} \mid \lambda_{X}^{\text {odd }} \in \Lambda_{n, d_{x}}^{\succ-} \lambda_{X}^{\text {even }} \in \Lambda_{n, d_{x}^{\prime}}^{\succ-}\right\}
$$

and

$$
\mathcal{S}=\left\{\lambda \in \Lambda_{2 n, d+d^{\prime}}^{\succ-} \mid \lambda^{o d d} \in \Lambda_{n, d^{\prime}}^{\succ-}, \lambda^{\text {even }} \in \Lambda_{n, d^{\prime}}^{\succ-}\right\}
$$


Recall that $X_{-}^{\lambda}$ is the scheme of $\Lambda_{2 n}^{\succ-}$-valued divisors on $X$ of degree $\lambda$. To a collection $\left(\lambda_{X}\right) \in \prod_{X} \mathcal{S}_{X}$ with $\lambda=\sum_{X} \lambda_{X}$, we attach a point $\tilde{D}=\sum_{X} \lambda_{X} X \in X_{-}^{\lambda}$. By Lemma 2.4.2, (41) identifies with

$$
\left.\underset{\lambda \in \mathcal{S}}{\oplus}\left(\lambda_{X}\right) \in \prod_{X} \mathcal{S}_{X}, \lambda=\sum_{X} \lambda_{X}\left(E_{-}^{\lambda}\right)_{\tilde{D}}\right)
$$

where $\tilde{D}$ is as above. Our claim follows.

\subsubsection{Proof of Proposition 2.4.4}

Combine Propositions 4.1.2 and 4.3.2.

\subsection{Proof of Theorem 4.3.9}

Let us sketch the ideas involved in the proof of Theorem 4.3.9. To prove part i), we stratify the stack $\mathrm{Sh}_{-}^{d, d^{\prime}}$ naturally and reduce to an estimation of dimension of the fibres of div_v intersected with strata. This, in turn, is reduced to Lemma 4.4.3, which is of local nature (the curve $X$ is replaced by a formal disk). Lemma 4.4.3 is proved in inductive way by introducing an ad hoc notion of a special transposition in the symmetric group (for a given element of $\Lambda_{2 m}$ ).

The more difficult part is Theorem 4.3.9 ii). Our approach here is to use another stratification of $\mathrm{Sh}_{-}^{d, d^{\prime}}$ given in Lemma 4.4.12. The definition of this second stratification is inspired by some constructions of Richardson and Springer from [17]. Write $\Sigma$ for the set of $d$-element subsets in $I=\left\{1, \ldots, d+d^{\prime}\right\}$. The corresponding strata are locally closed substacks

$$
\mathcal{X}_{\mathcal{J}} \cap \mathcal{Y}_{\mathcal{J}^{\prime}} \subset \mathrm{Sh}_{-}^{d, d^{\prime}}
$$

indexed by pairs $\mathcal{J}, \mathcal{J}^{\prime} \in \Sigma$. The key result is then Proposition 4.4.15. It affirms that $\mathcal{X}_{\mathcal{J}} \cap \mathcal{Y}_{\mathcal{J}^{\prime}}$ does not contribute to the desired highest direct image unless $\mathcal{J} \cap \mathcal{J}^{\prime}=\emptyset$ and some additional condition (C) holds. Under the latter conditions, it provides a further stratification of $\mathcal{X}_{\mathcal{J}} \cap \mathcal{Y}_{\mathcal{J}^{\prime}}$ and identifies the contribution of these strata.

Combining the above with general Remarks 4.4.13 and 2.4.6, we finish the proof of Theorem 4.3.9.

\subsection{1}

Recall the scheme $X_{+}^{\mu}$ defined in Section 1.1.1. For $\mu \in \Lambda_{m, d}^{\succ+}$ denote by $i_{\mu}: X_{+}^{\mu} \rightarrow \operatorname{Sh}_{0}^{d}$ the map sending $\left(D_{1}, \ldots, D_{m}\right)$ to $\mathcal{O}_{D_{1}} \oplus \ldots \oplus \mathcal{O}_{D_{m}}$. The image of $i_{\mu}$ is a locally closed substack 
of $\mathrm{Sh}_{0}^{d}$ denoted $\mathrm{Sh}_{0, \mu}^{d}$. As $\mu$ ranges in $\Lambda_{m, d}^{\succ+}$, the stacks $\mathrm{Sh}_{0, \mu}^{d}$ form a stratification of the open substack $\leq m \mathrm{Sh}_{0}^{d}$.

\subsubsection{Proof of Theorem 4.3.9 i)}

The argument is similar to ([9], Theorem 3.3.1). The stack $\mathrm{Sh}_{-}^{d, d^{\prime}}$ classifies: $F \in \mathrm{Sh}_{0}^{d}, F^{\prime} \in$ $\operatorname{Sh}_{0}^{d^{\prime}}$ with $D^{\prime}=\operatorname{div}\left(F^{\prime}\right) \geq D=\operatorname{div}(F)$, and a complete flag $0=F_{0} \subset F_{1} \subset \ldots \subset F_{d+d^{\prime}}=$ $F \oplus F^{\prime}$ of torsion subsheaves on $X$. Given $m \geq 1, \mu \in \Lambda_{m, d}^{\succ+}$ and $\mu^{\prime} \in \Lambda_{m, d^{\prime}}^{\succ+}$ set

$$
\mathrm{Sh}_{\mu, \mu^{\prime},-}=\mathrm{Sh}_{-}^{d, d^{\prime}} \times \operatorname{Sh}_{0}^{d} \times \operatorname{Sh}_{0}^{d^{\prime}}\left(\mathrm{Sh}_{0, \mu}^{d} \times \mathrm{Sh}_{0, \mu^{\prime}}^{d^{\prime}}\right)
$$

The composition

$$
\operatorname{Sh}_{\mu, \mu^{\prime},-} \rightarrow\left(X_{+}^{\mu} \times X_{+}^{\mu^{\prime}}\right) \times_{X^{(d)} \times X^{\left(d^{\prime}\right)}} V_{-}^{d, d^{\prime}} \stackrel{\mathrm{pr}}{\rightarrow} V_{-}^{d, d^{\prime}}
$$

is the restriction of $\operatorname{div}_{-}^{v}$.

Fix a $k$-point of $\left(X_{+}^{\mu} \times X_{+}^{\mu^{\prime}}\right) \times_{X^{(d)} \times X^{\left(d^{\prime}\right)}} V_{-}^{d, d^{\prime}}$ given by $\left(D, D^{\prime},\left(x_{i}\right)\right) \in V_{-}^{d, d^{\prime}, \nu}$, so $D \leq D^{\prime}$,

$$
\sum_{i=1}^{d+d^{\prime}} x_{i}=D+D^{\prime}
$$

and $\left(D_{j}\right) \in X_{+}^{\mu},\left(D_{j}^{\prime}\right) \in X_{+}^{\mu^{\prime}}$ with $D=\sum_{j} D_{j}$ and $D^{\prime}=\sum_{j} D_{j}^{\prime}$. Write $Y$ for the fibre of the first map in (43) over this point. We check that $\operatorname{dim} Y \leq-d^{\prime}$. Besides, by Lemma 4.4.3, if $m=1, \mu=(d), \mu^{\prime}=\left(d^{\prime}\right)$ then $\operatorname{dim} Y=-d^{\prime}$.

One has

$$
F \stackrel{\sim}{\rightarrow} \mathcal{O}_{D_{1}} \oplus \ldots \oplus \mathcal{O}_{D_{m}} \quad \text { and } \quad F^{\prime} \stackrel{\Im}{\rightarrow} \mathcal{O}_{D_{1}^{\prime}} \oplus \ldots \oplus \mathcal{O}_{D_{m}^{\prime}}
$$

As in ([9], Theorem 3.3.1), one checks that

$$
\operatorname{dim} \operatorname{Aut}(F)=\sum_{i=1}^{m}\left(\mu_{i}-\mu_{i+1}\right) i^{2} \quad \text { and } \quad \operatorname{dim} \operatorname{Aut}\left(F^{\prime}\right)=\sum_{i=1}^{m}\left(\mu_{i}^{\prime}-\mu_{i+1}^{\prime}\right) i^{2}
$$

We have canonically $F \stackrel{\overbrace{}}{\rightarrow} \oplus_{X \in X} F_{X^{\prime}}, F^{\prime} \stackrel{\rightarrow}{\rightarrow} \oplus_{X \in X} F_{X^{\prime}}^{\prime}$, where $F_{X^{\prime}}, F_{X}^{\prime}$ are torsion sheaves supported on a formal neighbourhood of $x$. Write

$$
D_{i}=\sum_{X \in X} \mu_{i, X} X \text { and } D_{i}^{\prime}=\sum_{X \in X} \mu_{i, X}^{\prime} X
$$


for $1 \leq i \leq m$. Set $d_{X}=\sum_{i} \mu_{i, X}$ and $d_{X}^{\prime}=\sum_{i} \mu_{i, X}^{\prime}$. Write $C F l\left(F_{X} \oplus F_{X}^{\prime}\right)$ for the scheme of complete flags on $F_{X} \oplus F_{X}^{\prime}$. We have

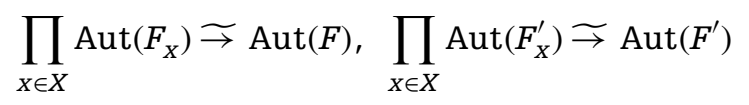

So,

$$
\left.Y \widetilde{\rightarrow} \prod_{X \in X}\left(C F l\left(F_{X} \oplus F_{X}^{\prime}\right)\right) /\left(\operatorname{Aut}\left(F_{X}\right) \times \operatorname{Aut}\left(F_{X}^{\prime}\right)\right)\right)
$$

By Lemma 4.4.3, for each $x \in X$,

$$
\left.\operatorname{dim}\left(\operatorname{CFl}\left(F_{X} \oplus F_{X}^{\prime}\right)\right) /\left(\operatorname{Aut}\left(F_{X}\right) \times \operatorname{Aut}\left(F_{X}^{\prime}\right)\right)\right) \leq-d_{X}^{\prime}
$$

Our claim follows.

Lemma 4.4.3. Let $m \geq 1, \mu \in \Lambda_{m, d^{\prime}}^{\succ+}, \mu^{\prime} \in \Lambda_{m, d^{\prime}}^{\succ+}$. Let $\mathcal{O}$ be a complete discrete valuation $k$-algebra. Write $\mathcal{O}_{m}=\mathcal{O} / \mathfrak{m}^{m}$, where $\mathfrak{m} \subset \mathcal{O}$ is the maximal ideal. $F=\mathcal{O}_{\mu_{1}} \oplus \ldots \oplus \mathcal{O}_{\mu_{m}}$, $F^{\prime}=\mathcal{O}_{\mu_{1}^{\prime}} \oplus \ldots \oplus \mathcal{O}_{\mu_{m}^{\prime}}$. Let $C F l\left(F \oplus F^{\prime}\right)$ be the scheme of complete flags of $\mathcal{O}$-modules on $F \oplus F^{\prime}$. Set

$$
\theta=\left(\mu_{1}^{\prime}, \mu_{1}, \mu_{2}^{\prime}, \mu_{2}, \ldots, \mu_{m}^{\prime}, \mu_{m}\right)
$$

Let $\eta \in \Lambda_{2 m, d+d^{\prime}}^{\succ+}$ be obtained from $\theta$ by permutation so that the resulting sequence is decreasing. Then

$$
\operatorname{dim} C F l\left(F \oplus F^{\prime}\right) /\left(\operatorname{Aut}(F) \times \operatorname{Aut}\left(F^{\prime}\right)\right) \leq-d^{\prime},
$$

and the inequality is strict unless $\theta=\eta$. In the latter case, (44) is an equality.

Proof. We have $F \oplus F^{\prime} \underset{\rightarrow}{\rightarrow} \mathcal{O}_{\eta_{1}} \oplus \ldots \oplus \mathcal{O}_{\eta_{2 m}}$. As in ([9], Theorem 3.3.1), one gets

$$
\operatorname{dim} C F l\left(F \oplus F^{\prime}\right)=\sum_{i=1}^{2 m}\left(\eta_{i}-\eta_{i+1}\right) \frac{i(i-1)}{2}
$$


and

$$
\operatorname{dim} \operatorname{Aut}(F)=\sum_{i=1}^{m}\left(\mu_{i}-\mu_{i+1}\right) i^{2}, \operatorname{dim} \operatorname{Aut}\left(F^{\prime}\right)=\sum_{i=1}^{m}\left(\mu_{i}^{\prime}-\mu_{i+1}^{\prime}\right) i^{2}
$$

Here $\mu_{m+1}=\mu_{m+1}^{\prime}=\eta_{2 m+1}=0$. One has

$$
\sum_{i=1}^{m}\left(\mu_{i}-\mu_{i+1}\right) i^{2}=\sum_{i=1}^{m} \mu_{i}(2 i-1) \quad \text { and } \quad \sum_{i=1}^{m}\left(\mu_{i}^{\prime}-\mu_{i+1}^{\prime}\right) i^{2}=\sum_{i=1}^{m} \mu_{i}^{\prime}(2 i-1)
$$

The expression (45) equals $\sum_{i=2}^{2 m} \eta_{i}(i-1)$. Set $\tau=(0,1, \ldots, 2 m-1) \in \Lambda_{2 m}$. We must show that $\langle\theta-\eta, \tau\rangle \geq 0$. In the case $\eta=\theta$, we get the desired equality.

Let $\mathcal{I}=\{1, \ldots, 2 m\}$. For a subset $J \subset \mathcal{I}$ with $|J|=m$ denote by $\theta_{J} \in \Lambda_{2 m}^{\succ}$ the element obtained as follows. Equip $J$ with the order induced from the standard order on $\mathcal{I}$, and similarly for $\mathcal{I}-J$. Then $\theta_{J}: \mathcal{I} \rightarrow \mathbb{Z}_{+}$is the map whose restriction to $J$ is $J \stackrel{\sim}{\rightarrow}\{1, \ldots, m\} \stackrel{\mu}{\rightarrow} \mathbb{Z}$, and whose restriction to $\mathcal{I}-J$ is $\mathcal{I}-J \underset{\rightarrow}{\sim}\{1, \ldots, m\} \stackrel{\mu^{\prime}}{\rightarrow} \mathbb{Z}_{+}$.

Let $\xi \in \Lambda_{2 m}^{\succ}$. If for some $1 \leq i<2 m$ we have $\xi_{i}<\xi_{i+1}$, let $\bar{\xi}$ be obtained from $\xi$ applying the permutation $(i, i+1)$. Then $\xi-\bar{\xi}=(0, \ldots, 0,-a, a, 0, \ldots, 0)$, where $a=\xi_{i+1}-\xi_{i}$ appears on $i+1$-th place. In this case, we say that $\bar{\xi}$ is obtained from $\xi$ by a special transposition. In this case $\langle\xi-\bar{\xi}, \tau\rangle=a>0$.

There is a finite sequence $\eta_{1}, \ldots, \eta_{s} \in \Lambda_{2 m}^{\succ}$ such that $\theta=\eta_{1}, \eta_{s}=\eta$, each $\eta_{i+1}$ is obtained from $\eta_{i}$ by a special transposition, and each $\eta_{i}$ is of the form $\theta_{J}$ for some $J$. On each step, the quantity $\left\langle\eta_{i}-\eta_{i+1}, \tau\right\rangle$ is strictly positive. So, $\langle\theta-\eta, \tau\rangle \geq 0$, and the inequality is strict unless $\theta=\eta$.

Remark by a referee: one has $\tau=\left(m-\frac{1}{2}\right)(1, \ldots, 1)-\rho_{\mathrm{GL}_{2 m}}$, so that $\langle\theta-\eta, \tau\rangle=$ $\left\langle\eta-\theta, \rho_{\mathrm{GL}_{2 m}}\right\rangle \geq 0$.

Remark 4.4.4. In the notations of the proof of Theorem 4.3.9 i), one has $\operatorname{dim} Y<-d^{\prime}$ unless

$$
D_{1}^{\prime} \geq D_{1} \geq D_{2}^{\prime} \geq D_{2} \geq \ldots \geq D_{m}^{\prime} \geq D_{m}
$$

Indeed, if $x \in X$ then

$$
F_{X} \stackrel{\sim}{\rightarrow} \mathcal{O}_{\mu_{1}, X} \oplus \ldots \oplus \mathcal{O}_{\mu_{m}, X^{\prime}}, \quad F_{X}^{\prime} \stackrel{\widetilde{\rightarrow}}{\rightarrow} \mathcal{O}_{\mu_{1}^{\prime}, X} \oplus \ldots \oplus \mathcal{O}_{\mu_{m}^{\prime}, X}
$$


and $\operatorname{deg}\left(F_{X}\right)=d_{X^{\prime}} \operatorname{deg}\left(F_{X}^{\prime}\right)=d_{X}^{\prime}$. Set $\theta_{X}=\left(\mu_{1, X^{\prime}}^{\prime} \mu_{1, X^{\prime}} \mu_{2, X^{\prime}}^{\prime} \mu_{2, X^{\prime}} \ldots, \mu_{m, X^{\prime}}^{\prime} \mu_{m, X}\right)$. Then $Y \widetilde{\Im} \prod_{X \in X} Y_{X}$ and $\operatorname{dim} Y_{X}<-d_{X}^{\prime}$ unless $\theta_{X} \in \Lambda_{2 m}^{\succ+}$. The condition (46) is equivalent to requiring that for any $X \in X, \theta_{X} \in \Lambda_{2 m}^{\succ+}$.

Remark 4.4.5. Let $m \geq 1, \mu \in \Lambda_{m, d}^{\succ+}, \mathcal{O}$ a complete discrete valuation $k$-algebra, $F=$ $\mathcal{O}_{\mu_{1}} \oplus \ldots \oplus \mathcal{O}_{\mu_{m}}$. Then, $\operatorname{CFl}(F) / \operatorname{Aut}(F)$ is of dimension $-\sum_{i=1}^{m} \mu_{i} i$.

4.4.6

Consider $\mu=(d) \in \Lambda_{1, d^{\prime}}^{\succ+} \mu^{\prime}=\left(d^{\prime}\right) \in \Lambda_{1, d^{\prime}}^{\succ+}$ for $m=1$. Consider the open substack $\mathrm{Sh}_{\mu, \mu^{\prime},-} \subset \mathrm{Sh}_{-}^{d, d^{\prime}}$ given by (42). Let

$$
{ }^{1} \operatorname{div}_{-}^{v}: \operatorname{Sh}_{\mu, \mu^{\prime},-} \rightarrow V_{-}^{d, d^{\prime}}
$$

be the restriction of $\operatorname{div}_{-}^{v}$. We get the natural map

$$
R^{-2 d^{\prime}}\left({ }^{1} \operatorname{div}_{-}^{v}\right) ! \overline{\mathbb{Q}}_{\ell} \rightarrow \mathrm{R}^{-2 d^{\prime}}\left(\operatorname{div}_{-}^{v}\right) ! \overline{\mathbb{Q}}_{\ell}
$$

It is not an isomorphism over the whole of $V_{-}^{d, d^{\prime}}$, as is seen from the proof of Theorem 4.3.9 i). However, the map (47) is an isomorphism over the open subscheme ${ }^{0} V_{-}^{d, d^{\prime}} \subset V_{-}^{d, d^{\prime}}$ defined in Section 4.3.6. It is easy to see that

$$
\mathrm{R}^{-2 d^{\prime}}\left(\operatorname{div}_{-}^{v}\right), \overline{\mathbb{Q}}_{\ell} \underset{\rightarrow}{\sim} \overline{\mathbb{Q}}_{\ell}
$$

over ${ }^{0} V_{-}^{d, d^{\prime}}$. Let

$$
{ }^{0}\left(X^{(d)} \times X^{\left(d^{\prime}-d\right)}\right) \subset X^{(d)} \times X^{\left(d^{\prime}-d\right)}
$$

be the open subscheme of $\left(D, D^{\prime}-D\right)$ such that $D$ is reduced, $D^{\prime}-D$ is reduced, and $\left(D^{\prime}-D\right) \cap D=\emptyset$.

To check the equivariance property of the isomorphism in Theorem 4.3.9 ii), it suffices to do it over ${ }^{0}\left(X^{(d)} \times X^{\left(d^{\prime}-d\right)}\right)$. Over this locus, it follows easily from the corresponding property of the Springer sheaf $\mathcal{S} p r_{E}^{2}$. In other words, this equivariance property in general is reduced to the case $d=d^{\prime}=1$. In the latter case, it is easy and left to a reader. 


\subsubsection{Stratifications I}

To prove Theorem 4.3.9 ii), we introduce a suitable stratification of $\mathrm{Sh}_{-}^{d, d^{\prime}}$. For this, we need some additional notations.

A point of $\mathrm{Sh}_{-}^{d, d^{\prime}}$ is written as $\left(F, F^{\prime},\left(F_{i}\right)\right)$, where $F_{1} \subset \ldots \subset F_{d+d^{\prime}}=F \oplus F^{\prime}$ is a complete flag of torsion subsheaves. We set $D=\operatorname{div}(F), D^{\prime}=\operatorname{div} F^{\prime}, x_{i}=\operatorname{div}\left(F_{i} / F_{i-1}\right)$ for $i \in I$.

Write $\Sigma$ for the set of $d$-element subsets in $I=\left\{1, \ldots, d+d^{\prime}\right\}$. Fix $\mathcal{J} \in \Sigma$. For a point of $\operatorname{Sh}_{-}^{d, d^{\prime}}$ set $\mathcal{F}_{i}^{\prime}=F^{\prime} \cap F_{i}$, so $\mathcal{F}_{i}^{\prime} \subset F_{i}$ is a subsheaf. Set $\bar{F}_{i}=F_{i} / \mathcal{F}_{i}^{\prime}$ for all $i$. We get a complete flag with repetitions

$$
\bar{F}_{1} \subset \bar{F}_{2} \subset \ldots \subset \bar{F}_{d+d^{\prime}}=F
$$

where we identify $\bar{F}_{j}$ with its image under the projection $\bar{F}_{j} \rightarrow\left(F \oplus F^{\prime}\right) / F^{\prime} \stackrel{\Im}{\rightarrow}$. We get also another complete flag with repetitions

$$
\mathcal{F}_{1}^{\prime} \subset \mathcal{F}_{2}^{\prime} \subset \ldots \subset \mathcal{F}_{d+d^{\prime}}^{\prime}=F^{\prime}
$$

We have the surjection $F_{j} \rightarrow \bar{F}_{j}$ for $j \in I$. So, for $j \in I$, we get a diagram

$$
\mathcal{F}_{j}^{\prime} / \mathcal{F}_{j-1}^{\prime} \hookrightarrow F_{j} / F_{j-1} \rightarrow \bar{F}_{j} / \bar{F}_{j-1},
$$

where the first map is the natural injection and the second is the natural surjection.

\section{4 .8}

Denote by $\mathcal{X}_{\mathcal{J}} \subset \mathrm{Sh}_{-}^{d, d^{\prime}}$ the locally closed substack given by the property that for $j \in \mathcal{J}$ one has $\mathcal{F}_{j}^{\prime}=\mathcal{F}_{j-1}^{\prime}$, so that $\bar{F}_{j} / \bar{F}_{j-1} \in \mathrm{Sh}_{0}^{1}$. For a point of $\mathcal{X}_{\mathcal{J}}$, the second map in (48) is an isomorphism for $j \in \mathcal{J}$,

$$
x_{j}=\operatorname{div}\left(\bar{F}_{j} / \bar{F}_{j-1}\right) \text { for } j \in \mathcal{J},
$$

and $D=\sum_{j \in \mathcal{J}} X_{j}$. For a point of $\mathcal{X}_{\mathcal{J}}$ we get $\mathcal{F}_{j}^{\prime} / \mathcal{F}_{j-1}^{\prime} \in \operatorname{Sh}_{0}^{1}$ for $j \in I-\mathcal{J}$, the first map in (48) is an isomorphism for $j \in I-\mathcal{J}$,

$$
x_{j}=\operatorname{div}\left(\mathcal{F}_{j}^{\prime} / \mathcal{F}_{j-1}^{\prime}\right) \text { for } j \in I-\mathcal{J}
$$

and $D^{\prime}=\sum_{j \in I-\mathcal{J}} X_{j}$ 


\section{4 .9}

For a point of $\mathrm{Sh}_{-}^{d, d^{\prime}}$ set similarly $\mathcal{F}_{i}=F \cap F_{i}$, so $\mathcal{F}_{i} \subset F_{i}$ is a subsheaf. Set $\bar{F}_{i}^{\prime}=F_{i} / \mathcal{F}_{i}$ for $i \in I$. We get a complete flag with repetitions

$$
\mathcal{F}_{1} \subset \mathcal{F}_{2} \subset \ldots \subset \mathcal{F}_{d+d^{\prime}}=F
$$

View $\bar{F}_{i}^{\prime}$ as a subsheaf of $F^{\prime}$ via the natural map $F_{i} / \mathcal{F}_{i} \rightarrow\left(F \oplus F^{\prime}\right) / F \Im F^{\prime}$. This gives a complete flag with repetitions

$$
\bar{F}_{1}^{\prime} \subset \bar{F}_{2}^{\prime} \subset \ldots \subset \bar{F}_{d+d^{\prime}}^{\prime}=F^{\prime}
$$

For a point of $\mathrm{Sh}_{-}^{d, d^{\prime}}$ and $j \in I$, we get a diagram

$$
\mathcal{F}_{j} / \mathcal{F}_{j-1} \hookrightarrow F_{j} / F_{j-1} \rightarrow \bar{F}_{j}^{\prime} / \bar{F}_{j-1}^{\prime},
$$

where the first map is the natural injection, the second is the natural surjection.

\subsubsection{0}

Denote by $\mathcal{Y}_{\mathcal{J}} \subset \mathrm{Sh}_{-}^{d, d^{\prime}}$ the locally closed substack given by the property that for $j \in \mathcal{J}$ one has $\mathcal{F}_{j} / \mathcal{F}_{j-1} \in \operatorname{Sh}_{0}^{1}$, so that $\bar{F}_{j}^{\prime}=\bar{F}_{j-1}^{\prime}$. For a point of $\mathcal{Y}_{\mathcal{J}}$ and $j \in \mathcal{J}$ the first map in (49) is an isomorphism, so

$$
x_{j}=\operatorname{div}\left(\mathcal{F}_{j} / \mathcal{F}_{j-1}\right) \text { for } j \in \mathcal{J}
$$

and $D=\sum_{j \in \mathcal{J}} x_{j}$. For a point of $\mathcal{Y}_{\mathcal{J}}$ and $j \in I-\mathcal{J}$ the second map in (49) is an isomorphism,

$$
\operatorname{div}\left(\bar{F}_{j}^{\prime} / \bar{F}_{j-1}^{\prime}\right)=x_{j} \quad \text { for } j \in I-\mathcal{J}
$$

and $D^{\prime}=\sum_{j \in I-\mathcal{J}} X_{j}$

\subsubsection{1}

On the sheaf $F^{\prime}$ we get two flags: $\left(\bar{F}_{j}^{\prime}\right)$ and $\left(\mathcal{F}_{j}^{\prime}\right)$. The relation between them is as follows. For any $k \in I$, we have the natural inclusion $\mathcal{F}_{k}^{\prime} \hookrightarrow F_{k}$ and a surjection $F_{k} \rightarrow F_{k} / \mathcal{F}_{k}=\bar{F}_{k}^{\prime}$. 
Their composition $\mathcal{F}_{k}^{\prime} \rightarrow \bar{F}_{k}^{\prime}$ is clearly injective for any $k$. We get the diagram

$$
\begin{array}{ccccccc}
\bar{F}_{1}^{\prime} & \subset & \bar{F}_{2}^{\prime} & \subset \ldots \subset & \bar{F}_{d+d^{\prime}}^{\prime} & =F^{\prime} \\
\cup & & \cup & & & \cup & \\
\mathcal{F}_{1}^{\prime} & \subset & \mathcal{F}_{2}^{\prime} & \subset \ldots \subset & \mathcal{F}_{d+d^{\prime}}^{\prime} & =F^{\prime}
\end{array}
$$

For a torsion sheaf $\mathcal{G}$ on $X$ write $\ell(\mathcal{G})$ for its length. For $k \in I$ we get $\ell\left(\mathcal{F}_{k}^{\prime}\right) \leq \ell\left(\bar{F}_{k}^{\prime}\right)$. For any $k \in I, \ell\left(\mathcal{F}_{k}^{\prime}\right)$ is the number of elements $1 \leq j \leq k$ such that $j \notin \mathcal{J}$. Besides, $\ell\left(\bar{F}_{k}^{\prime}\right)$ is the number of elements $1 \leq j \leq k$ such that $j \notin \mathcal{J}^{\prime}$. For $k \in I$ set

$$
\mathcal{J}_{k}=\mathcal{J} \cap\{1, \ldots, k\}, \quad \mathcal{J}_{k}^{\prime}=\mathcal{J}^{\prime} \cap\{1, \ldots, k\}
$$

We see that the pair $\mathcal{J}, \mathcal{J}^{\prime}$ satisfies the following condition:

(C) if $k \in I$ then $\left|\mathcal{J}_{k}^{\prime}\right| \leq\left|\mathcal{J}_{k}\right|$.

Similarly, on $F$, we get two flags $\left(\bar{F}_{j}\right)$ and $\left(\mathcal{F}_{j}\right)$. The relation between them is similar. For $k \in I$, we have an injection $\mathcal{F}_{k} \hookrightarrow F_{k}$ and a surjection $F_{k} \rightarrow \bar{F}_{k}$. Their composition $\mathcal{F}_{k} \rightarrow \bar{F}_{k}$ is injective. We get the diagram

$$
\begin{array}{ccccccc}
\bar{F}_{1} & \subset & \bar{F}_{2} & \subset \ldots \subset & \bar{F}_{d+d^{\prime}} & =F \\
\cup & & \cup & & & \cup & \\
\mathcal{F}_{1} & \subset & \mathcal{F}_{2} & \subset \ldots \subset & \mathcal{F}_{d+d^{\prime}} & =F
\end{array}
$$

The property that for any $k \in I, \ell\left(\mathcal{F}_{k}\right) \leq \ell\left(\bar{F}_{k}\right)$ is nothing but $(\mathrm{C})$. We summarize this discussion in the following.

Lemma 4.4.12. The stack $\mathrm{Sh}_{-}^{d, d^{\prime}}$ is stratified by locally closed substacks $\mathcal{X}_{\mathcal{J}} \cap \mathcal{Y}_{\mathcal{J}^{\prime}}$ indexed by pairs $\left(\mathcal{J}, \mathcal{J}^{\prime}\right) \in \Sigma^{2}$ such that (C) holds for $\left(\mathcal{J}, \mathcal{J}^{\prime}\right)$. In particular, if $i \in \mathcal{J}$ is the smallest element, $j^{\prime} \in \mathcal{J}^{\prime}$ is the biggest element then $\mathcal{J} \cup \mathcal{J}^{\prime} \subset\left\{i, \ldots, j^{\prime}\right\}$.

Remark 4.4.13. If $f: \mathcal{Z} \rightarrow \mathcal{Z}^{\prime}$ is a morphism of finite type between algebraic stacks, $F$ is a smooth $\overline{\mathbb{Q}}_{\ell}$-sheaf on $\mathcal{Z}$, assume all the fibres of $f$ are of dimension $\leq d$. Assume $\mathcal{Z}$ is stratified by locally closed substacks $i_{a}: \mathcal{Z}^{a} \hookrightarrow \mathcal{Z}$ indexed by $a \in \mathcal{A}$. Let $f^{a}: \mathcal{Z}^{a} \rightarrow \mathcal{Z}^{\prime}$ be the restriction of $f$. Then $\mathrm{R}^{2 d} f_{!} F$ admits a filtration by subsheaves with the successive quotients being $\mathrm{R}^{2 d} f_{!}^{a} i_{a}^{*} F, a \in \mathcal{A}$. We refer to $\mathrm{R}^{2 d} f_{!}^{a} i_{a}^{*} F$ as the contribution of the stratum $\mathcal{Z}^{a}$ to the highest direct image $\mathrm{R}^{2 d} f_{!} F$. 


\subsubsection{Stratifications II}

Recall the subset Inv $\subset S_{I}$ defined in Section 4.3.6. An involution $w \in \operatorname{Inv}$ writes as a disjoint product of two cycles $w=\left(i_{1}, j_{1}\right)\left(i_{2}, j_{2}\right) \ldots\left(i_{r}, j_{r}\right)$ with $i_{k}<j_{k}$ for $1 \leq k \leq r$. As in ([17], Section 5.3), for such an involution $w$ set $\operatorname{Hi}(w)=\left\{j_{1}, \ldots, j_{r}\right\}$ and $\operatorname{Lo}(w)=\left\{i_{1}, \ldots, i_{r}\right\}$. As in loc.cit., we call $\mathrm{Hi}(w)$ (resp., Lo(w)) the high points (resp., low points) of $w$.

Proposition 4.4.15. i) For $\mathcal{J}, \mathcal{J}^{\prime} \in \Sigma$ the stratum $\mathcal{X}_{\mathcal{J}} \cap \mathcal{Y}_{\mathcal{J}^{\prime}}$, does not contribute to

$$
R^{-2 d^{\prime}}\left(\operatorname{div}_{-}^{v}\right) \overline{\mathbb{Q}}_{\ell}
$$

unless $\mathcal{J} \cap \mathcal{J}^{\prime}=\emptyset$ and the condition (C) holds.

ii) Assume $\mathcal{J} \cap \mathcal{J}^{\prime}=\varnothing$ and the condition (C). Then $\mathcal{X}_{\mathcal{J}} \cap \mathcal{Y}_{\mathcal{J}^{\prime}}$ admits a stratification by locally closed substacks $\mathcal{X}^{w}$ indexed by $w \in \operatorname{Inv}$ such that $\operatorname{Hi}(w)=\mathcal{J}^{\prime}, \operatorname{Lo}(w)=\mathcal{J}$.

For such $w$ let $\alpha: I \rightarrow \bar{I}$ be the unique element of $\mathcal{E}$ such that the classes of the corresponding equivalence relation on $I$ are precisely the $W$-orbits. The composition $\mathcal{X}^{W} \hookrightarrow \mathrm{Sh}_{-}^{d, d^{\prime}} \rightarrow V_{-}^{d, d^{\prime}}$ factors uniquely through the closed subscheme norm $_{\alpha}: V_{\alpha} \hookrightarrow$ $V_{-}^{d, d^{\prime}}$, and the contribution of $\mathcal{X}^{w}$ to $(51)$ is $\left(\text { norm }_{\alpha}\right)_{!} \overline{\mathbb{Q}}_{\ell}$.

Lemma 4.4.16. Let $F_{1}, F_{2} \subset F$ be torsion sheaves on $X$ and $\tilde{F}$ be the coproduct of the diagram $F_{1} \leftarrow F_{1} \cap F_{2} \rightarrow F_{2}$. Then, the induced map $\tilde{F} \rightarrow F$ is injective.

\subsubsection{Proof of Proposition 4.4.15}

ii) Consider two subsets $\mathcal{J}, \mathcal{J}^{\prime} \in \Sigma$ such that $\mathcal{J} \cap \mathcal{J}^{\prime}=\emptyset$, and the condition (C) holds. Set $\overline{\mathcal{J}}=\{1, \ldots, d\}$. Equip $\mathcal{J}$ (resp., $\mathcal{J}^{\prime}$ ) with the natural order: $i_{1} \leq i_{2}$ if $i_{1}$ is less of equal to $i_{2}$. These orders are linear, and so yield bijections that we denote

$$
b: \overline{\mathcal{J}} \Im \mathcal{J}, \quad b^{\prime}: \overline{\mathcal{J}} \Im \mathcal{J}^{\prime}
$$

If $i \in \overline{\mathcal{J}}$ then $\ell\left(\bar{F}_{b(i)}\right)=i$. If $j \in \overline{\mathcal{J}}$ then $\ell\left(\mathcal{F}_{b^{\prime}(j)}\right)=j$.

Denote by ${ }_{d}^{d}$ the set of matrices $e=\left(e_{i}^{j}\right)$ with $i, j \in \overline{\mathcal{J}}, e_{i}^{j} \in \mathbb{Z}_{+}$. For a point of $\mathcal{X}_{\mathcal{J}} \cap \mathcal{Y}_{\mathcal{J}^{\prime}}$ define the matrix $e \in{ }_{d}^{d}$ by

$$
\sum_{k=1}^{i} \sum_{l=1}^{j} e_{k}^{l}=\ell\left(\bar{F}_{b(i)} \cap F_{b^{\prime}(j)}\right)=\ell\left(\bar{F}_{b(i)} \cap \mathcal{F}_{b^{\prime}(j)}\right)
$$

for $i, j \in \overline{\mathcal{J}}$. From Lemma 4.4.16, one gets by induction that indeed $e_{i}^{j} \in \mathbb{Z}_{+}$for any $i, j \in \overline{\mathcal{J}}$. 
The matrix $e$ has the properties:

- $e_{i}^{j} \in\{0,1\}$.

- If $j \in \overline{\mathcal{J}}$ then $\sum_{k=1}^{d} e_{k}^{j}=1$. If $i \in \overline{\mathcal{J}}$ then $\sum_{j=1}^{d} e_{i}^{j}=1$. So, 1 appears precisely once in each row (resp., each column) of $e$.

We get a unique $\bar{w} \in S_{d}$ such that $e_{\bar{w} j}^{j}=1$ for all $j \in \overline{\mathcal{J}}$, and $e_{i}^{j}=0$ unless $i=\bar{w} j$. Define the unique involution $w \in \operatorname{Inv}$ by the properties:

P1) $\left.\quad w\right|_{\mathcal{J}^{\prime}}=b \bar{w}\left(b^{\prime}\right)^{-1}$;

P2) $\quad I-\left(\mathcal{J} \cup \mathcal{J}^{\prime}\right)$ is the set of fixed points of $w$.

Conversely, given an involution $w \in \operatorname{Inv}$ with $\operatorname{Hi}(w)=\mathcal{J}^{\prime}, \operatorname{Lo}(w)=\mathcal{J}$, there is a unique $\bar{W} \in S_{d}$ satisfying the property P1). Define the locally closed substack $\mathcal{X}^{w} \subset \mathcal{X}_{\mathcal{J}} \cap \mathcal{Y}_{\mathcal{J}^{\prime}}$ by fixing the corresponding $\bar{w} \in S_{d}$, or equivalently, the corresponding matrix $e$ satisfying (52). This is the desired stratification of $\mathcal{X}_{\mathcal{J}} \cap \mathcal{Y}_{\mathcal{J}^{\prime}}$ by the locally closed substacks $\mathcal{X}^{w}$.

Denote by $f^{W}: \mathcal{X}^{W} \rightarrow\left(\operatorname{Sh}_{0}^{1}\right)^{I-\mathcal{J}^{\prime}}$ the map sending $\left(F, F^{\prime},\left(F_{i}\right)\right)$ to the collection $\left(F_{i} / F_{i-1}\right)_{i \in I-\mathcal{J}^{\prime}}$. This is a generalized affine fibration of rank zero. For $i \in I-\mathcal{J}^{\prime}$, we then let $x_{i}=\operatorname{div}\left(F_{i} / F_{i-1}\right)$. For $i \in \mathcal{J}^{\prime}$, we let $x_{i}=x_{w i}$.

As we have seen in Section 4.4.7, for a point of $\mathcal{X}_{\mathcal{J}}$ one has $D=\sum_{j \in \mathcal{J}} x_{j}$ and $D^{\prime}=\sum_{j \in I-\mathcal{J}} x_{j}$. So, the composition $\mathcal{X}^{w} \hookrightarrow \mathrm{Sh}_{-}^{d, d^{\prime}} \rightarrow V_{-}^{d, d^{\prime}}$ factors uniquely through the closed subscheme $V_{\alpha} \hookrightarrow V_{-}^{d, d^{\prime}}$, and the contribution of $\mathcal{X}^{w}$ to $\mathrm{R}^{-2 d^{\prime}}\left(\operatorname{div}_{-}^{v}\right) \overline{\mathbb{Q}}_{\ell}$ is $\left(\text { norm }_{\alpha}\right)_{!} \overline{\mathbb{Q}}_{\ell}$.

i) This is obtained from the dimension estimates and is left to a reader. If $\mathcal{J} \cap \mathcal{J}^{\prime} \neq \emptyset$, and (C) holds, one may also stratify $\mathcal{X}_{\mathcal{J}} \cap \mathcal{Y}_{\mathcal{J}^{\prime}}$ by fixing the relative position of the two flags $\left(\bar{F}_{i}\right),\left(\mathcal{F}_{j}\right)$ on $F$ as in the case $\mathcal{J} \cap \mathcal{J}^{\prime}=\emptyset$.

Remark 4.4.18. In the situation of Proposition 4.4.15 ii) for certain $\bar{w} \in S_{d}$ the stack $\mathcal{X}^{W}$ is empty. For example, consider the case $d=d^{\prime}=2, \mathcal{J}=\{1,3\}, \mathcal{J}^{\prime}=\{2,4\}$. Then $\mathcal{X}_{\mathcal{J}} \cap \mathcal{Y}_{\mathcal{J}^{\prime}}=\mathcal{X}^{w}$, where $w=(12)(34)$. It corresponds to $\bar{w}=\mathrm{id} \in S_{2}$. Indeed, in this case, the stratification is given by the relative position of the two subsheaves $\bar{F}_{1}, \mathcal{F}_{2} \in \mathrm{Sh}_{0}^{1}$ in $F \in \mathrm{Sh}_{0}^{2}$. However, from the diagram (50), we know that $\mathcal{F}_{2} \subset \bar{F}_{2}$, and for a point of $\mathcal{X}_{\mathcal{J}} \cap \mathcal{Y}_{\mathcal{J}}$, we have $\bar{F}_{1}=\bar{F}_{2}$ by definition. So, $\mathcal{F}_{2}=\bar{F}_{1}$ for any point of $\mathcal{X}_{\mathcal{J}} \cap \mathcal{Y}_{\mathcal{J}^{\prime}}$. In general, if $\mathcal{X}^{w} \neq \emptyset$, then $w$ has to be compatible with the diagram (50).

For the convenience of the reader, we give several examples of the stratifications of $\mathrm{Sh}_{-}^{d, d^{\prime}}$ appeared in Section 4.4.7 and Proposition 4.4.15. 


\subsubsection{Example $d=1, d^{\prime}>1$}

This is a first nontrivial example. We have $\ell(F)=1, \ell\left(F^{\prime}\right)=d^{\prime}$. Let $\mathcal{J}^{\prime}=\{j\}, \mathcal{J}=\{i\}$. The substack $\mathcal{X}_{\mathcal{J}} \cap \mathcal{Y}_{\mathcal{J}^{\prime}} \subset \operatorname{Sh}_{-}^{d, d^{\prime}}$ is given by the properties:

- $F_{i-1} \subset F^{\prime}, F_{i} \cap F^{\prime}=F_{i-1}$,

- $F \cap F_{j-1}=0, F \subset F_{j}$.

(The first conditions guarantee that $F \cap F_{i-1}=0$, so if $F \subset F_{j}$ then $j \geq i$ ).

Let $\overline{\mathcal{X}}$ be the stack classifying $F \in \mathrm{Sh}_{0}^{1}, F^{\prime} \in \operatorname{Sh}_{0}^{d^{\prime}}$ with $\operatorname{div}(F) \leq \operatorname{div}\left(F^{\prime}\right)$ and a complete flag $\left(F_{1}^{\prime} \subset \ldots F_{d^{\prime}}^{\prime}\right)$ on $F^{\prime}$. For each $1 \leq i \leq d^{\prime}+1$ we get an isomorphism

$$
\overline{\mathcal{X}} \underset{\rightarrow}{\rightarrow} \mathcal{X}_{\mathcal{J}} \cap \mathcal{Y}_{\mathcal{J}}
$$

sending $\left(F, F^{\prime},\left(F_{j}^{\prime}\right)\right)$ to $\left(F, F^{\prime},\left(F_{j}\right)\right)$, where $\left(F_{j}\right)$ is the complete flag on $F \oplus F^{\prime}$ given by

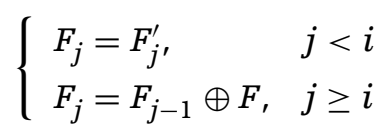

The composition $\overline{\mathcal{X}} \underset{\rightarrow}{\rightarrow} \mathcal{X}_{\mathcal{J}} \cap \mathcal{Y}_{\mathcal{J}} \stackrel{\operatorname{div}_{-}^{v}}{\rightarrow} V_{-}^{d, d^{\prime}}$ sends $\left(F, F^{\prime},\left(F_{j}^{\prime}\right)\right)$ to the collection $x=\operatorname{div} F, D^{\prime}=$ $\operatorname{div} F^{\prime}$ and $\left(x_{1}, \ldots, x_{i-1}, x, x_{i}, \ldots, x_{d^{\prime}}\right)$, where $x_{i}=\operatorname{div}\left(F_{i}^{\prime} / F_{i-1}^{\prime}\right)$. Using Remark 4.4.5, one sees that the contribution of the stratum $\mathcal{X}_{\mathcal{J}} \cap \mathcal{Y}_{\mathcal{J}}$ to $\mathrm{R}^{-2 d^{\prime}}\left(\operatorname{div}_{-}^{v}\right)$ ! $\overline{\mathbb{Q}}_{\ell}$ is zero.

Assume $1 \leq i<j \leq d^{\prime}+1$. Let $w=(i j) \in \operatorname{Inv}$ then $\mathcal{X}_{\mathcal{J}} \cap \mathcal{Y}_{\mathcal{J}^{\prime}}=\mathcal{X}^{w}$. The map $f^{W}: \mathcal{X}^{W} \rightarrow\left(\mathrm{Sh}_{0}^{1}\right)^{I-\mathcal{J}^{\prime}}$ is a generalized affine fibration. Indeed, taking the quotient by $F_{i-1}$ and replacing $F^{\prime}$ by $F_{j}$, this claim is reduced to the special case $i=1, j=d^{\prime}+1$. In the latter case, the map $f^{W}$ is described as follows.

Lemma 4.4.20. Let $d=1, d^{\prime}>1, i=1, j=d^{\prime}+1$ and $w=(i j)$. The stack $\mathcal{X}^{w}$ classifies: $F, F_{1} \in \mathrm{Sh}_{0}^{1}$, an isomorphism $F \Im F_{1}$, a complete flag $\mathcal{F}_{2}^{\prime} \subset \ldots \subset \mathcal{F}_{d^{\prime}}^{\prime}$ of torsion sheaves on $\mathcal{F}_{d^{\prime}}^{\prime} \in \mathrm{Sh}_{0}^{d^{\prime}-1}$ as in Section 4.4.7. So, $\ell\left(\mathcal{F}_{k}^{\prime}\right)=k-1$ for $2 \leq k \leq d^{\prime}$. Here for $2 \leq i \leq d^{\prime}$ one has canonically

$$
F_{i} / F_{i-1} \stackrel{\sim}{\rightarrow} \mathcal{F}_{i}^{\prime} / \mathcal{F}_{i-1}^{\prime}
$$

Besides, $F \underset{\rightarrow}{\rightarrow} F_{1} \underset{\rightarrow}{\rightarrow} F_{d^{\prime}+1} / F_{d^{\prime}}$

Proof. The stack $\mathcal{X}_{\mathcal{J}} \cap \mathcal{Y}_{\mathcal{J}^{\prime}}$ is given by two conditions: $F_{1} \cap F^{\prime}=0$ and $F \cap F_{d^{\prime}}=0$. Using the notations from Section 4.4.7, we get $F_{k}=F_{1} \oplus \mathcal{F}_{k}^{\prime}$ for $2 \leq k \leq d^{\prime}$, and $F_{d^{\prime}+1}=F_{d^{\prime}} \oplus F$. So, $F \oplus F_{1} \oplus \mathcal{F}_{d^{\prime}}^{\prime}=F \oplus F^{\prime}$. Consider the natural projection

$$
F^{\prime}=\mathcal{F}_{d^{\prime}+1}^{\prime} \rightarrow\left(F \oplus F_{1} \oplus \mathcal{F}_{d^{\prime}}^{\prime}\right) / \mathcal{F}_{d^{\prime}}^{\prime} \underset{\rightarrow}{\rightarrow} \oplus F_{1}
$$


Both its components are nonzero. Thus, the induced maps $\mathcal{F}_{d^{\prime}+1}^{\prime} / \mathcal{F}_{d^{\prime}}^{\prime} \rightarrow F_{1}$ and $\mathcal{F}_{d^{\prime}+1}^{\prime} / \mathcal{F}_{d^{\prime}}^{\prime} \rightarrow F$ are both nonzero, hence isomorphisms. This defines the desired isomorphism $F \underset{\rightarrow}{\rightarrow} F_{1}$, and $F^{\prime}$ as the unique subsheaf in $F \oplus F_{1} \oplus \mathcal{F}_{d^{\prime}}^{\prime}$ containing $\mathcal{F}_{d^{\prime}}^{\prime}$ and mapping diagonally to $F \oplus F_{1}$.

\subsubsection{Example $d=d^{\prime}=2$}

Only the strata $\mathcal{X}_{\{1,3\}} \cap \mathcal{Y}_{\{2,4\}}$ and $\mathcal{X}_{\{1,2\}} \cap \mathcal{Y}_{\{3,4\}}$ contribute to (51). One gets $\mathcal{X}_{\{1,3\}} \cap \mathcal{Y}_{\{2,4\}}=$ $\mathcal{X}^{W}$ for $w=(12)(34)$ as in Remark 4.4.18. The locus $\mathcal{X}_{\{1,2\}} \cap \mathcal{Y}_{\{3,4\}}$ consists of two strata $\mathcal{X}^{w}, \mathcal{X}^{w^{\prime}}$ for $w=(13)(24)$ and $w^{\prime}=(14)(23)$. The substack $\mathcal{X}^{w} \subset \mathcal{X}_{\{1,2\}} \cap \mathcal{Y}_{\{3,4\}}$ is given by $\bar{F}_{1} \subset F_{3}$, and $\mathcal{X}^{W^{\prime}}$ is its complement in $\mathcal{X}_{\{1,2\}} \cap \mathcal{Y}_{\{3,4\}}$.

The stack $\mathcal{X}^{w}$ classifies: $F_{1} \in \mathrm{Sh}_{0}^{1}, F_{2} / F_{1} \in \mathrm{Sh}_{0}^{1}$ with divisors $x_{1}, x_{2}$, for which we set $x_{3}=x_{1}, x_{4}=x_{2}$, and an extension $0 \rightarrow F_{1} \rightarrow F_{2} \rightarrow F_{2} / F_{1} \rightarrow 0$ on $X$.

The stack $\mathcal{X}^{w^{\prime}}$ classifies: $\bar{F}_{1}, \mathcal{F}_{3} \in \operatorname{Sh}_{0}^{1}$ with $x_{1}=\operatorname{div}\left(\bar{F}_{1}\right), x_{2}=\operatorname{div}\left(\mathcal{F}_{3}\right)$, for which we set $x_{3}=x_{2}, x_{4}=x_{1}$. So, the map $f^{w^{\prime}}: \mathcal{X}^{w^{\prime}} \rightarrow\left(\operatorname{Sh}_{0}^{1}\right)^{2}$ sending the above point to $\left(F_{1}, F_{2} / F_{1}\right)$ is an isomorphism.

\subsubsection{End of the proof of Theorem 4.3.9}

The normalization of $V_{-}^{d, d^{\prime}}$ is described in Section 4.3.6. We first stratify $S_{-}^{d, d^{\prime}}$ by locally closed substacks $\mathcal{X}_{\mathcal{J}} \cap \mathcal{Y}_{\mathcal{J}^{\prime}}$ indexed by pairs $\mathcal{J}, \mathcal{J}^{\prime} \in \Sigma$. By Section 4.4.7, this locus is empty unless the condition (C) holds. By Proposition 4.4.15, only the strata with $\mathcal{J} \cap \mathcal{J}^{\prime}=\emptyset$ contribute to (51). For $\mathcal{J}, \mathcal{J}^{\prime} \in \Sigma$ with $\mathcal{J} \cap \mathcal{J}^{\prime}=\emptyset$ such that (C) holds, we stratify $\mathcal{X}_{\mathcal{J}} \cap \mathcal{Y}_{\mathcal{J}^{\prime}}$ by locally closed substacks $\mathcal{X}^{w}$ as in Proposition 4.4.15 indexed by $w \in \operatorname{Inv}$ such that $\operatorname{Hi}(w)=\mathcal{J}^{\prime}, \operatorname{Lo}(w)=\mathcal{J}$. Let $\alpha: I \rightarrow \bar{I}$ be the surjection such that the classes of the corresponding equivalence relation on $I$ are precisely $W$-orbits. By Proposition 4.4 .15 , the contribution of the stratum $\mathcal{X}^{W}$ to $(51)$ identifies with $\left(\text { norm }_{\alpha}\right)_{!} \overline{\mathbb{Q}}_{\ell}$. Now $\left(\text { norm }_{\alpha}\right)_{!} \overline{\mathbb{Q}}_{\ell}\left[d^{\prime}\right]$ is an irreducible perverse sheaf. So, $\left(\mathrm{R}^{-2 d^{\prime}}\left(\operatorname{div}_{-}^{v}\right)_{!} \overline{\mathbb{Q}}_{\ell}\right)\left[d^{\prime}\right]$ is perverse and admits a filtration with the associated graded

$$
\underset{\alpha \in \mathcal{E}}{\oplus}\left(\operatorname{norm}_{\alpha}\right) \overline{\mathbb{Q}}_{\ell}\left[d^{\prime}\right] \underset{\rightarrow}{\longrightarrow} \operatorname{IC}\left(V_{-}^{d, d^{\prime}}\right)
$$

Any such filtration splits canonically into a direct sum, because the summands are irreducible perverse sheaves supported on different irreducible components (cf. Remark 2.4.6).

Finally, the proof of Proposition 2.3.5 is complete. So, Theorem 2.1.2 is proved. 


\subsection{Comparison with [17]}

Section 4.5 is not used in the paper and may be skipped. We include it to give another description of the stratification used in Proposition 4.4.15. Consider the stack $\mathcal{D}$ classifying vector spaces $V, V^{\prime}$ of dimensions $d, d^{\prime}$ and a complete flag $V_{1} \subset \ldots \subset$ $V_{d+d^{\prime}}=V \oplus V^{\prime}$ of vector subspaces. The set of isomorphism classes of $\mathcal{D}$ is finite and was described in [17]. In this section, we introduce a morphism $f: \operatorname{Sh}_{-}^{d, d^{\prime}} \rightarrow \mathcal{D}$ and a stratification of $\mathcal{D}$ such that the stratification of $\mathrm{Sh}_{-}^{d, d^{\prime}}$ used in Proposition 4.4.15 is obtained as the pullback by $f$.

\subsection{1}

Consider the stack $\mathcal{D}$ classifying vector spaces $V, V^{\prime}$ of dimensions $d, d^{\prime}$ and a complete flag $V_{1} \subset \ldots \subset V_{d+d^{\prime}}=V \oplus V^{\prime}$ of vector subspaces. The set of points of $\mathcal{D}$ is finite and described in ([17], Section 5.3). It is naturally in bijection with

$$
\overline{\mathcal{E}}=\{(w, \mathcal{J}) \in \operatorname{Inv} \times \Sigma \mid \operatorname{Hi}(w) \subset \mathcal{J}, \operatorname{Lo}(w) \cap \mathcal{J}=\emptyset\}
$$

Consider the map $f: \operatorname{Sh}_{-}^{d, d^{\prime}} \rightarrow \mathcal{D}$ sending $\left(F, F^{\prime},\left(F_{i}\right)\right)$ to the collection $\left(V, V^{\prime},\left(V_{i}\right)\right)$ with $V=\mathrm{H}^{0}(X, F), V^{\prime}=\mathrm{H}^{0}\left(X, F^{\prime}\right)$, and $V_{i}=\mathrm{H}^{0}\left(X, F_{i}\right)$. There is a stratification of $\mathcal{D}$ whose preimage under $f$ gives the stratification of $\mathrm{Sh}_{-}^{d, d^{\prime}}$ that we used in Section 4.4 .7 and Proposition 4.4.15. However, this is not the stratification by the classes of isomorphisms of points of $\mathcal{D}$. We give some details for the convenience of the reader.

We define stratifications of $\mathcal{D}$ by locally closed substacks $\mathcal{D}^{\mathcal{J}}$ with $\mathcal{J} \in \Sigma$ (resp., by locally closed substacks $\mathcal{D}_{\mathcal{J}}$ with $\mathcal{J} \in \Sigma$ ) such that $f^{-1}\left(\mathcal{D}^{\mathcal{J}}\right)=\mathcal{Y}_{\mathcal{J}}$ and $f^{-1}\left(\mathcal{D}_{\mathcal{J}}\right)=\mathcal{X}_{\mathcal{J}}$ for any $\mathcal{J} \in \Sigma$.

\section{5 .2}

If we pick bases $\left\{e_{1}, \ldots, e_{d}\right\}$ in $V$ and $\left\{e_{d+1}, \ldots, e_{d+d^{\prime}}\right\}$ in $V^{\prime}$, then we get a complete flag in $k^{d+d^{\prime}}$ given by some $g B_{d+d^{\prime}} \in \mathrm{GL}_{d+d^{\prime}} / B_{d+d^{\prime}}$. Set $K=\mathrm{GL}_{d} \times \mathrm{GL}_{d^{\prime}}$. Forgetting these bases, one gets an isomorphism $\mathcal{D} \underset{\rightarrow}{\rightarrow} \backslash \mathrm{GL}_{d+d^{\prime}} / B_{d+d^{\prime}}$ with the the stack quotient.

The variety of decompositions $k^{d+d^{\prime}}=\oplus_{i=1}^{d+d^{\prime}} W_{i}$ into one-dimensional vector subspaces identifies naturally with $\mathrm{GL}_{d+d^{\prime}} / T_{d+d^{\prime}}$. Here $T_{d+d^{\prime}}$ is the torus of diagonal matrices. Namely, to $g T_{d+d^{\prime}}$ we associate $W_{i}=g \operatorname{Vect}\left(e_{i}\right)$.

If $V, V^{\prime}$ are vector spaces of dimensions $d, d^{\prime}$ and $\oplus_{i=1}^{d+d^{\prime}} W_{i}=V \oplus V^{\prime}$ is a decomposition into one-dimensional subspaces, it gives rise to a torus $T_{W}=\{g \in$ $\left.\mathrm{GL}\left(V \oplus V^{\prime}\right) \mid g\left(W_{i}\right)=W_{i}\right\}$ for any $i$. Let $\theta$ be the automorphism of $V \oplus V^{\prime}$ acting as 1 
on $V$ and as -1 on $V^{\prime}$. The torus $T_{W}$ is $\theta$-stable if $\theta T_{W} \theta^{-1}=T_{W}$. This means that there is an involution $w \in S_{d+d^{\prime}}$ such that $\theta\left(W_{i}\right)=W_{w i}$ for any $i$.

Write $\widetilde{\mathcal{D}}$ for the stack classifying vector spaces $V, V^{\prime}$ of dimensions $d, d^{\prime}$, and a decomposition into one-dimensional vector subspaces $V \oplus V^{\prime}=\oplus_{i=1}^{d+d^{\prime}} W_{i}$. If we pick bases of $V, V^{\prime}$ as above, a point of $\widetilde{\mathcal{D}}$ together with these bases gives an element of $\mathrm{GL}_{d+d^{\prime}} / T_{d+d^{\prime}}$. Forgetting the bases, one gets an isomorphism with the stack quotient $K \backslash \mathrm{GL}_{d+d^{\prime}} / T_{d+d^{\prime}} \underset{\rightarrow}{\rightarrow}$.

Denote by $\overline{\mathcal{D}} \subset \widetilde{\mathcal{D}}$ the closed substack given by the property that $T_{W}$ is $\theta$-stable. For an involution $w \in S_{d+d^{\prime}}$ denote by $\overline{\mathcal{D}}^{w} \subset \overline{\mathcal{D}}$, the locally closed substack given by the property that $\theta\left(W_{i}\right)=W_{w i}$ for any $i \in I$. This is a stratification of $\overline{\mathcal{D}}$ indexed by the set Inv of involutions in $S_{d+d^{\prime}}$.

Let $c \in \mathrm{GL}_{d+d^{\prime}}$ be the automorphism acting as 1 on $\operatorname{Vect}\left(e_{1}, \ldots, e_{d}\right)$ and as -1 on $\operatorname{Vect}\left(e_{d+1}, \ldots, e_{d+d^{\prime}}\right)$. Let $\theta_{c}$ be the inner automorphism of $\mathrm{GL}_{d+d^{\prime}}$ sending $g$ to $\operatorname{cgc}^{-1}$. Then $K=\left(\mathrm{GL}_{d+d^{\prime}}\right)^{\theta_{c}}$. As in ([17], Section 1.2), set

$$
\mathcal{V}=\left\{g \in \mathrm{GL}_{d+d^{\prime}} \mid g^{-1} \theta_{C}(g) \in N_{d+d^{\prime}}\right\}
$$

where $N_{d+d^{\prime}}$ is the normalizer of $T_{d+d^{\prime}}$ in $\mathrm{GL}_{d+d^{\prime}}$. Then, $\mathcal{V}$ is stable under the action of $T_{d+d^{\prime}}$ by right translations. Now

$$
\mathcal{V} / T_{d+d^{\prime}} \subset \mathrm{GL}_{d+d^{\prime}} / T_{d+d^{\prime}}
$$

is the closed subvariety of those decompositions $k^{d+d^{\prime}} \stackrel{\rightarrow}{\rightarrow} \oplus_{i=1}^{d+d^{\prime}} W_{i}$ for which $T_{W} \subset$ $\mathrm{GL}_{d+d^{\prime}}$ is $\theta_{c}$-stable. Note that $\mathcal{V}$ is stable under the action of $K$ by left translations. Picking bases of $V, V^{\prime}$ as above, this gives an isomorphism with the stack quotient

$$
K \backslash \mathcal{V} / T_{d+d^{\prime}} \stackrel{\sim}{\rightarrow} \overline{\mathcal{D}}
$$

For $x \in N_{d+d^{\prime}}, w \in S_{d+d^{\prime}}$ the image of $x$ in $S_{d+d^{\prime}}$ is $w$ if for any $i \in I, x \operatorname{Vect}\left(e_{i}\right)=$ $\operatorname{Vect}\left(e_{w i}\right)$. Let $N_{d+d^{\prime}}^{W} \subset N_{d+d^{\prime}}$ be the closed subscheme of $x \in N_{d+d^{\prime}}$ over $w$. Let also $\mathcal{V}^{W} \subset \mathcal{V}$ be the closed subscheme given by requiring that $g^{-1} \theta_{c}(g) \in N_{d+d^{\prime}}^{W}$. Then (53) restricts for any $w \in \operatorname{Inv}$ to an isomorphism

$$
K \backslash \mathcal{V}^{W} / T_{d+d^{\prime}} \stackrel{\sim}{\rightarrow} \overline{\mathcal{D}}^{W}
$$

By ([17], Proposition 1.2.2), number of points of $\overline{\mathcal{D}}$ and $\mathcal{D}$ is finite. Consider the map $\widetilde{\mathcal{D}} \rightarrow \mathcal{D}$ sending $\left(V, V^{\prime},\left(W_{i}\right)\right)$ to $\left(V, V^{\prime},\left(V_{i}\right)\right)$, where $V_{i}=\oplus_{j=1}^{i} W_{j}$. Let $f_{\mathcal{D}}: \overline{\mathcal{D}} \rightarrow \mathcal{D}$ be 
its restriction to $\overline{\mathcal{D}}$. Now ([17], Proposition 1.2.2) implies that $f_{\mathcal{D}}$ induces a bijection on the set of points of $\overline{\mathcal{D}}$ and $\mathcal{D}$.

For $w \in \operatorname{Inv}$, the stack $\overline{\mathcal{D}}^{w}$ has a finite number of points, this number could be bigger than one. For $w \in \operatorname{Inv}$ set $\mathcal{D}^{w}=f_{\mathcal{D}}\left(\overline{\mathcal{D}}^{w}\right)$ viewed a locally closed substack.

\subsection{3}

Let $P \subset \mathrm{GL}_{d+d^{\prime}}$ be the parabolic preserving $\operatorname{Vect}\left(e_{1}, \ldots, e_{d}\right)$ in $k^{d+d^{\prime}}$. So, $K$ is the standard Levi of $P$, and $B_{d+d^{\prime}} \subset P$. Let $\mathcal{D}_{P}$ be the stack classifying a complete flag of vector spaces $V_{1} \subset \ldots \subset V_{d+d^{\prime}}$ and a subspace $V \subset V_{d+d^{\prime}}$. As above, we get an isomorphism

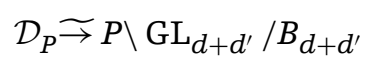

Let $\eta_{P}: \mathcal{D} \rightarrow \mathcal{D}_{P}$ be the map forgetting $V^{\prime}$.

Set $\overline{\mathcal{J}}=\{1, \ldots, d\} \subset I$. Let $W_{\overline{\mathcal{J}}} \subset W=S_{d+d^{\prime}}$ be the stabilizer of $\overline{\mathcal{J}}$. We have a bijection $W / W_{\overline{\mathcal{J}}} \widetilde{\rightarrow} \Sigma$ sending $w W_{\overline{\mathcal{J}}} \in W / W_{\overline{\mathcal{J}}}$ to $W \mathcal{J} \in \Sigma$. The set of points of $\mathcal{D}_{P}$ is in bijection with $\Sigma$. Namely, to $w W_{\overline{\mathcal{J}}} \in W / W_{\overline{\mathcal{J}}}$ with $\mathcal{J}=w \overline{\mathcal{J}} \in \Sigma$, we associate $P w^{-1} B_{d+d^{\prime}}$. Write $\mathcal{D}_{P}^{\mathcal{J}}$ for the corresponding locally closed substack of $\mathcal{D}_{P}$. For $\mathcal{J} \in \Sigma$ let $\mathcal{D}^{\mathcal{J}}$ be the preimage of $\mathcal{D}_{P}^{\mathcal{J}}$ under $\eta_{P}$.

According to ([17], Section 5.3), for $w \in \operatorname{In} v, \mathcal{J} \in \Sigma$ the stack $\mathcal{D}^{w, \mathcal{J}}=\mathcal{D}^{w} \cap \mathcal{D}^{\mathcal{J}}$ is either empty or has precisely one point. Moreover, it is nonempty iff

$$
\operatorname{Hi}(w) \subset \mathcal{J}, \operatorname{Lo}(w) \cap \mathcal{J}=\emptyset
$$

This way one gets a bijection between the set of points of $\mathcal{D}$ and $\overline{\mathcal{E}}$. For $\mathcal{J} \in \Sigma$ one has a stratification

$$
\mathcal{D}^{\mathcal{J}}=\underset{(W, \mathcal{J}) \in \overline{\mathcal{E}}}{\sqcup} \mathcal{D}^{w, \mathcal{J}}
$$

\subsection{4}

For $w \in S_{d+d^{\prime}}$ write $\operatorname{Fix}(w)$ for the set of fixed points of $w$ on $I$. For $w \in \operatorname{Inv}, \mathcal{J} \in \Sigma$ let $\mathcal{D}^{W, \mathcal{J}}$ be nonempty. Then, the unique point of $\mathcal{D}^{W, \mathcal{J}}$ admits the following presentation $\left(V, V^{\prime},\left(V_{i}\right)\right)$. There is a decomposition $V \oplus V^{\prime}=\oplus_{i \in I} W_{i}$ with $\theta\left(W_{i}\right)=W_{w i}$ for all $i$ with the properties:

- $\quad V_{i}=W_{1} \oplus \ldots \oplus W_{i}$ for all $i \in I$

- if $j \in \operatorname{Fix}(w) \cap \mathcal{J}$ then $W_{j} \subset V$;

- if $j \in \operatorname{Fix}(w)-\mathcal{J}$ then $W_{j} \subset V^{\prime}$. 
In addition, let $1 \leq i<j \leq d+d^{\prime}$ with $w(i)=j$. Pick $0 \neq w_{i} \in w_{i}$, let $w_{j}=\theta\left(w_{i}\right)$. Then $w_{i} \oplus W_{j}$ is $\theta$-stable with a base $\left\{w_{i}+w_{j}, w_{i}-w_{j}\right\}$. Here $w_{i}+w_{j} \in V, w_{i}-w_{j} \in V^{\prime}$.

\section{5 .5}

Consider also the parabolic $P^{-} \subset \mathrm{GL}_{d+d^{\prime}}$ preserving $\operatorname{Vect}\left(e_{d+1}, \ldots, e_{d+d^{\prime}}\right)$ in $k^{d+d^{\prime}}$. Let $\mathcal{D}_{P^{-}}$be the stack classifying a complete flag of vector spaces $\left(V_{1} \subset \ldots \subset V_{d+d^{\prime}}\right)$ and a subspace $V^{\prime} \subset V_{d+d^{\prime}}$. We have a projection $\eta_{P^{-}}: \mathcal{D} \rightarrow \mathcal{D}_{P^{-}}$forgetting $V$. As above, we have an isomorphism with the stack quotient $\mathcal{D}_{P^{-}} \widetilde{\rightarrow} P^{-} \backslash \mathrm{GL}_{d+d^{\prime}} / B_{d+d^{\prime}}$.

For $w W_{\overline{\mathcal{J}}} \in W / W_{\overline{\mathcal{J}}}$ with $\mathcal{J}=w \overline{\mathcal{J}} \in \Sigma$ the orbit $P^{-} w^{-1} B_{d+d^{\prime}}$ defines a locally closed substack $\mathcal{D}_{P^{-}}^{\mathcal{J}} \subset \mathcal{D}_{P^{-}}$. Write $\mathcal{D}_{\mathcal{J}}$ for the preimage of $\mathcal{D}_{P^{-}}^{\mathcal{J}}$ under $\eta_{P^{-}}$.

For $w \in \operatorname{Inv}, \mathcal{J} \in \Sigma$ set $\mathcal{D}_{\mathcal{J}}^{W}=\mathcal{D}^{w} \cap \mathcal{D}_{\mathcal{J}}$. One checks that if $\mathcal{D}_{\mathcal{J}}^{W}$ is nonempty then it consists of one point. Moreover, for $w \in \operatorname{Inv}, \mathcal{J} \in \Sigma$ the stack $\mathcal{D}_{\mathcal{J}}^{W}$ is nonempty iff

$$
\operatorname{Hi}(w) \cap \mathcal{J}=\emptyset, \operatorname{Lo}(w) \subset \mathcal{J}
$$

\section{Proof of Theorem 2.2.2}

5.0 .1

We have the diagram extending (7)



where $q_{\mathcal{Z}}$ sends $\left(\Omega^{2 n-1} \hookrightarrow L, L^{\prime}\right)$ to $\left(L, L^{\prime}\right)$, and $q_{2 n}$ sends $\left(\Omega^{2 n-1} \hookrightarrow M\right)$ to $M$.

Let $\mathcal{U}_{2 n} \subset \operatorname{Bun}_{2 n}$ be the open substack given by the property that $\operatorname{Ext}^{1}\left(\Omega^{2 n-1}, M\right)=$ 0 for $M \in \operatorname{Bun}_{2 n}$. Let $\mathcal{U}_{2 n}^{n} \subset \operatorname{Bun}_{n}$ be the open substack given by $\operatorname{Ext}^{1}\left(\Omega^{2 n-1}, L\right)=0$ for $L \in \operatorname{Bun}_{n}$. For $d \in \mathbb{Z}$ let $\operatorname{Bun}_{2 n, d} \subset \operatorname{Bun}_{2 n}$ be the component given by $\operatorname{deg} M=$ $d+\operatorname{deg}\left(\Omega^{(2 n-1)+\ldots+1}\right)$ for $M \in \operatorname{Bun}_{2 n}$. So, $q_{2 n}: \mathcal{M}_{2 n, d} \rightarrow \operatorname{Bun}_{2 n, d}$.

5.0 .2

Assume $d+d^{\prime}$ large enough, so that $\mathrm{Aut}_{E}$ over $\mathrm{Bun}_{2 n, d+d^{\prime}}$ is the extension by zero under $\mathcal{U}_{2 n} \hookrightarrow \operatorname{Bun}_{2 n}$. Indeed, by ([2], Lemma 3.3), any $M \in \operatorname{Bun}_{2 n, d+d^{\prime}}$ not lying in $\mathcal{U}_{2 n}$ is very unstable in the sense of loc.cit., and the $*$-fibre of Aut $_{E}$ at $M$ vanishes. If $\left(L, L^{\prime}\right) \in v_{n}^{-1}\left(\mathcal{U}_{2 n}\right)$ then $L \in \mathcal{U}_{2 n}^{n}$. So, we get an exact triangle

$$
\left(q_{\mathcal{Z}}\right)_{!} \overline{\mathbb{Q}}_{\ell} \rightarrow \overline{\mathbb{Q}}_{\ell}[-2 M] \rightarrow \overline{\mathbb{Q}}_{\ell}
$$


over $v_{n}^{-1}\left(\mathcal{U}_{2 n}\right) \cap(\operatorname{det} \times \operatorname{det})^{-1}\left((\operatorname{Pic} X \times \operatorname{Pic} X)^{d, d^{\prime}}\right)$, here $M=d+(g-1)\left(n-2 n^{2}\right)$ is the relative dimension of $q_{\mathcal{Z}}$ over $\mathcal{Z}_{n}^{d, d^{\prime}}$. This yields an exact triangle

$$
\pi_{!} v_{\mathcal{Z}}^{*} \mathcal{K}_{2 n, E}\left[\operatorname{dim} . \operatorname{rel}\left(v_{\mathcal{Z}}\right)+M\right] \rightarrow \operatorname{Per}_{E}^{d, d^{\prime}} \rightarrow \operatorname{Per}_{E}^{d, d^{\prime}}[2 M]
$$

on $(\operatorname{Pic} X \times \operatorname{Pic} X)^{d, d^{\prime}}$.

If $d>d^{\prime}$ and $d$ is large enough then, by Theorem 2.1.2, $\pi_{!} \nu_{\mathcal{Z}}^{*} \mathcal{K}_{2 n, E}$ vanishes. Under these assumptions then (54) shows that $\operatorname{Per}_{E}^{d, d^{\prime}}=0$. Using the $S_{2}$-symmetry of $P e r_{E}$ and the equivariance property (8), one obtains i).

\section{0 .3}

From i) and the equivariance property (8), one gets $N \in \mathbb{Z}$ such that for any $d, d^{\prime} \in \mathbb{Z}$, $\operatorname{Per}_{E}^{d, d^{\prime}}$ is placed in usual degrees $\leq N$. The second claim follows now from the exact triangle (54) and Theorem 2.1.2. Theorem 2.2.2 is proved.

\section{Proof of Theorem 2.6.2}

6.0 .1

Let $P \subset \mathbb{G}$ be the Siegel parabolic. Then $\operatorname{Bun}_{P}$ is the stack classifying $L \in \operatorname{Bun}_{2}, \mathcal{A} \in \operatorname{Bun}_{1}$ and an exact sequence $0 \rightarrow \operatorname{Sym}^{2} L \rightarrow ? \rightarrow \mathcal{A} \rightarrow 0$ on $X$ (cf. Remark 6.0.3). Let $\mathcal{S}_{P}$ be the stack classifying $L \in \operatorname{Bun}_{2}, \mathcal{A} \in \operatorname{Bun}_{1}$ and a section $s: \operatorname{Sym}^{2} L \rightarrow \mathcal{A} \otimes \Omega$ on $X$. Let $h: \operatorname{Bun}_{1} \times \operatorname{Bun}_{1} \rightarrow \mathcal{S}_{P}$ be the map sending $\left(L_{1}, L_{2}\right)$ to $L=L_{1} \oplus L_{2}$ with the natural symmetric form $\operatorname{Sym}^{2} L \rightarrow \mathrm{L}_{1} \otimes L_{2}=\mathcal{A} \otimes \Omega$, so $L_{i}$ are isotropic in $L$.

One gets the diagram

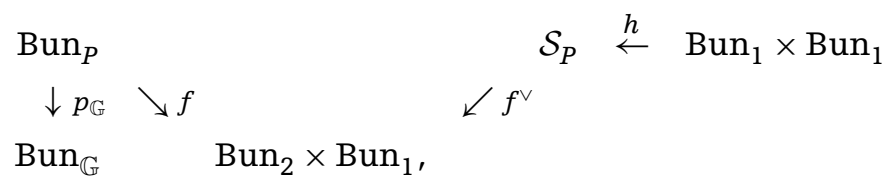

where $f, f^{\vee}$ are the projections sending the above points to $(L, \mathcal{A})$, and $p_{\mathbb{G}}$ is the extension of scalars map. The maps $f, f^{\vee}$ are dual generalized vector bundles, we denote by Four $_{\psi}$ : $\mathrm{D}^{\prec}\left(\operatorname{Bun}_{P}\right) \rightarrow \mathrm{D}^{\prec}\left(\mathcal{S}_{P}\right)$ the corresponding Fourier transform.

Recall that $\mathbb{H} \underset{\mathrm{G}}{\rightarrow} \mathrm{GL}_{4} / \mu_{2}$. Set $R=\left(\mathrm{GL}_{2} \times \mathrm{GL}_{2}\right) / \mu_{2}$, where $\mu_{2}$ is included diagonally. So, $R$ is a subgroup of $\mathbb{H}$. The homomorphism $\operatorname{det} \times \operatorname{det}: \mathrm{GL}_{2} \times \mathrm{GL}_{2} \rightarrow \mathbb{G}_{m} \times \mathbb{G}_{m}$ factors through $R \rightarrow \mathbb{G}_{m} \times \mathbb{G}_{m}$. Consider the diagram

$$
\operatorname{Bun}_{\mathbb{H}} \stackrel{\alpha}{\leftarrow} \operatorname{Bun}_{R} \stackrel{\beta}{\rightarrow} \operatorname{Bun}_{1} \times \operatorname{Bun}_{1} \stackrel{\varrho \times \varrho}{\rightarrow} \operatorname{Bun}_{1} \times \operatorname{Bun}_{1}
$$


where $\alpha, \beta$ are the corresponding extension of scalars maps, and $\varrho: \operatorname{Bun}_{1} \rightarrow \operatorname{Bun}_{1}$ sends $\mathcal{B}$ to $\mathcal{B}^{*} \otimes \Omega$.

The following is established in ([10], Proposition 11).

Proposition 6.0.2. For any $F \in \mathrm{D}^{-}\left(\mathrm{Bun}_{\mathbb{H}}\right)$ ! there is an isomorphism (up to a cohomological shift)

$$
h^{*} \operatorname{Four}_{\psi}\left(p_{\mathbb{G}}^{*} F_{\mathbb{G}}(F)\right) \stackrel{\Im}{\rightarrow}(\varrho \times \varrho)_{!} \beta_{!} \alpha^{*} F
$$

The following diagram commutes, where the square is Cartesian

$$
\begin{aligned}
& \mathrm{Bun}_{4} \stackrel{\bar{\alpha}}{\leftarrow} \mathrm{Bun}_{2} \times \mathrm{Bun}_{2}
\end{aligned}
$$

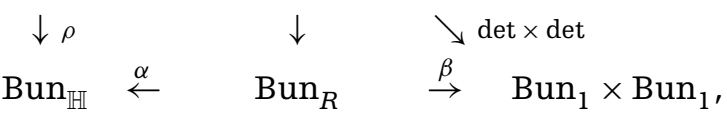

and the vertical arrows are the natural extension of scalars maps.

By Proposition 6.0.2, it suffices to show that $\beta_{!} \alpha^{*} K_{E^{*}, \chi^{*}, \mathbb{H}}$ is not zero. By

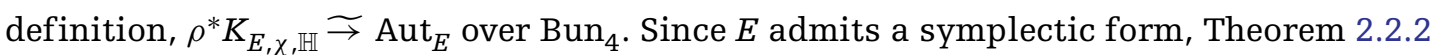
implies that $(\operatorname{det} \times \operatorname{det})_{!} \bar{\alpha}^{*} \mathrm{Aut}_{E}$ is nonzero on $\mathrm{Bun}_{1} \times \mathrm{Bun}_{1}$. This concludes the proof of Theorem 2.6.2.

Remark 6.0.3. Let $S$ be a scheme. Assume given a semidirect product $P=M \rtimes U$ of affine group schemes on $S$, where $U \subset P$ is a normal closed subgroup, $M$ acts on $U$ by conjugation. Then the groupoid of $P$-torsors on $S$ is canonically equivalent to the groupoid of pairs: an $M$-torsor $\mathcal{F}_{M}$ on $S$, and a $U_{\mathcal{F}_{M}}$-torsor on $S$. Here $U_{\mathcal{F}_{M}}$ is the group scheme on $S$, the twist of $U$ by the $M$-torsor $\mathcal{F}_{M}$.

\section{A Antistandard Levi subgroups in reductive groups}

\section{A.0.1}

In this section, we introduce a notion of an antistandard Levi subgroup $M$ in a reductive group G. Our purpose is to prove Propositions A.0.5 and A.2.2. The first one is a property of the restriction of spherical perverse sheaves under $\mathrm{Gr}_{M} \rightarrow \mathrm{Gr}_{G}$ for such Levi subgroup 
$M$. The second one is an application of the Casselman-Shalika formula from [2] for such Levi subgroups.

\section{A.0.2}

Work over an algebraically closed field $k$. Let $\mathcal{O}=k[[t]] \subset k((t))=F$. Let $G$ be a connected reductive group over $k, T \subset B \subset G$ a maximal torus and Borel, $\Lambda$ the coweights lattice $\Lambda^{+}$the dominant coweights. Let $M$ be a Levi with $T \subset M, \Lambda_{M}^{+}$be the dominant coweights for $M, \Lambda_{M}^{\text {pos }}$ the $\mathbb{Z}_{+}$-span of simple coroots of $M$. Let $U \subset B$ be the unipotent radical, $B_{M}=B \cap M$. Let $U_{M} \subset B_{M}$ be the unipotent radical. Write $W$ (resp., $W_{M}$ ) for the Weyl group of $G$ (resp., of $M$ ). Our choices of $B$ and $B_{M}$ yield choices of positive coroots for $G$ and $M$. Write $W_{0}$ (resp., $W_{0}^{M}$ ) for the longest element of $W$ (resp., of $W_{M}$ ). Let $\check{\rho}$ be the half sum of positive roots for $G$, write $\leq$ for the standard order on $\Lambda$. We denote by $\check{\rho}_{M}$ and $\leq_{M}$ for the corresponding objects for $M$. We ignore the Tate twists everywhere.

We do not assume that $M$ is standard, so a simple coroot of $M$ is not necessarily simple for $G$.

Definition A.0.3. Say that $M$ is antistandard if for any simple coroot $\alpha$ of $M$ one has $\left\langle\alpha, \check{\rho}-\check{\rho}_{M}\right\rangle>0$. So, such an $\alpha$ is a positive coroot for $G$ but not simple.

\section{A.0.4}

For $\lambda \in \Lambda$ write $S^{\lambda}$ for the $U(F)$-orbit on $\mathrm{Gr}_{G}$ through $t^{\lambda}, S_{M}^{\lambda}$ is the $U_{M}(F)$-orbit on $\mathrm{Gr}_{M}$ through $t^{\lambda}$. For $v \in \Lambda^{+}$we write $\mathrm{Gr}_{G}^{v}$ for the corresponding $G(\mathcal{O})$-orbit, $\mathcal{A}_{v}$ for the corresponding $G(\mathcal{O})$-equivariant perverse sheaf on $\mathrm{Gr}_{G}$. Let $i: \mathrm{Gr}_{M} \rightarrow \mathrm{Gr}_{G}$ be the natural map. Write $i_{\lambda}:$ Spec $k \rightarrow \mathrm{Gr}_{M}$ for the point $t^{\lambda}$.

Proposition A.0.5. Let $\lambda \in \Lambda, v \in \Lambda^{+}$. Then 1) the complex $\mathrm{R} \Gamma_{C}\left(S_{M}^{\lambda}, i^{*} \mathcal{A}_{v}\right)$ is placed in degrees $\leq\langle\lambda, 2 \check{\rho}\rangle$.

2) Assume in addition that $M$ is antistandard. Then the above inequality is strict unless $\lambda \in-\Lambda_{G}^{+}$and $v=w_{0}(\lambda)$. In the latter case, the natural map

$$
\mathrm{R} \Gamma_{C}\left(S_{M}^{\lambda}, i^{*} \mathcal{A}_{v}\right) \rightarrow i_{\lambda}^{*} i^{*} \mathcal{A}_{v} \underset{\rightarrow}{\widetilde{Q}} \overline{\mathbb{Q}}_{\ell}[-\langle\lambda, 2 \check{\rho}\rangle]
$$

is an isomorphism.

\section{A.0.6 Question}

For future research we raise the following question. In the situation of Proposition A.0.5, describe the top cohomology group of $\mathrm{R} \Gamma_{C}\left(S_{M}^{\lambda}, i^{*} \mathcal{A}_{v}\right)$ in the degree $\langle\lambda, 2 \check{\rho}\rangle$ in general. 


\section{A.1 Proof of Proposition A.0.5}

\section{A.1.1}

Now we will parameterize the spherical orbits in $\mathrm{Gr}_{M}$ which intersect both the support of $\mathcal{A}_{v}$ and the semi-infinite orbit $S_{M}^{\lambda}$ and thereby obtain the set $\mathcal{I}(\lambda, v)$ below.

For $\lambda, v$ as in Proposition A.0.5 write $\mathcal{I}(\lambda, v)$ for the set of $\mu \in \Lambda_{M}^{+}$such that for any $w \in W_{M}, w \lambda \leq_{M} \mu$, and for any $w \in W, w \mu \leq \nu$. Let $f: \mathcal{I}(\lambda, \nu) \rightarrow \mathbb{Z}$ be the function

$$
f(\mu)=\left\langle\mu, 2 \check{\rho}_{M}\right\rangle-\left\langle\mu^{\prime}, 2 \check{\rho}\right\rangle,
$$

where $\mu^{\prime} \in \Lambda^{+}$is the unique element lying in $W \mu$. We derive Proposition A.0.5 from the following.

Proposition A.1.2. Let $\lambda \in \Lambda, v \in \Lambda^{+}$. Then for any $\mu \in \mathcal{I}(\lambda, v)$ one has

$$
f(\mu) \leq\left\langle\lambda, 2 \check{\rho}-2 \check{\rho}_{M}\right\rangle
$$

Assume in addition $M$ antistandard. Then the above inequality is strict unless $\lambda \in-\Lambda_{G^{\prime}}^{+}$ $w_{0}^{M}(\lambda)=\mu$ and $\mu^{\prime}=w_{0}(\lambda)$.

\section{A.1.3 Proof of Proposition A.0.5}

Let $\bar{\lambda}$ be the image of $\lambda$ in $\pi_{1}(M), \mathrm{Gr}_{M}^{\bar{\lambda}}$ the corresponding connected component of $\mathrm{Gr}_{M}$. Consider the scheme $Y \subset \mathrm{Gr}_{M}^{\bar{\lambda}}$, which is a union of $M(\mathcal{O})$-orbits for $\mu \in \mathcal{I}(\lambda, v)$ (the scheme structure is not important here). Note that $Y \subset \mathrm{Gr}_{M}^{\bar{\lambda}} \times{ }_{\mathrm{Gr}_{G}} \overline{\mathrm{Gr}}_{G}^{v}$. Let $\bar{Y}$ be the closure of $Y$ in $\mathrm{Gr}_{M}^{\bar{\lambda}}$. Let $j: Y \hookrightarrow \bar{Y}$ be the natural immersion.

We claim that the $*$-restriction $\left.\mathcal{A}_{v}\right|_{Y}$ is placed in perverse degrees $\leq\left\langle\lambda, 2 \check{\rho}-2 \check{\rho}_{M}\right\rangle$. In addition, if $M$ is antistandard, this inequality is strict unless $\lambda \in-\Lambda_{G}^{+}$and $\nu=w_{0}(\lambda)$.

To see this, we must show that for $\mu \in \mathcal{I}(\lambda, \nu),\left.\mathcal{A}_{v}\right|_{\mathrm{Gr}_{M}^{\mu}}$ is placed in usual degrees $\leq\left\langle\lambda, 2 \check{\rho}-2 \check{\rho}_{M}\right\rangle-\left\langle\mu, 2 \check{\rho}_{M}\right\rangle$. Besides, if $M$ is antistandard, this inequality is strict unless $\lambda \in-\Lambda_{G}^{+}$and $v=w_{0}(\lambda)$. Let $\mu^{\prime} \in W \mu$ such that $\mu^{\prime} \in \Lambda^{+}$. Then $\left.\mathcal{A}_{v}\right|_{\mathrm{Gr}_{G}^{\mu^{\prime}}}$ is placed in usual degrees $\leq-\left\langle\mu^{\prime}, 2 \check{\rho}\right\rangle$, and the inequality is strict unless $\mu^{\prime}=v$. So, our claim follows from Proposition A.1.2.

Consider now $j_{!}\left(\left.\mathcal{A}_{v}\right|_{Y}\right)$, it is placed in perverse degrees $\leq\left\langle\lambda, 2 \check{\rho}-2 \check{\rho}_{M}\right\rangle$ over $\mathrm{Gr}_{M}$. Besides, if $M$ is antistandard, this inequality is strict unless $\lambda \in-\Lambda_{G}^{+}$and $v=w_{0}(\lambda)$. So, by ([15], Lemma 3.9), $\mathrm{R} \Gamma_{C}\left(S_{M}^{\lambda}, j_{!}\left(\left.\mathcal{A}_{v}\right|_{Y}\right)\right)$ is placed in degrees $\leq\langle\lambda, 2 \check{\rho}\rangle$. Now

$$
\mathrm{R} \Gamma_{C}\left(S_{M}^{\lambda}, j_{!}\left(\left.\mathcal{A}_{v}\right|_{Y}\right)\right) \stackrel{\mathrm{R}}{\rightarrow} \Gamma_{C}\left(S_{M}^{\lambda}, i^{*} \mathcal{A}_{v}\right)
$$

because $S_{M}^{\lambda} \cap(\bar{Y}-Y)=\emptyset$ by ([15], Theorem 3.2). Proposition A.0.5 is proved. 


\section{A.1.4 Proof of Proposition A.1.2}

Let $\mu \in \mathcal{I}(\lambda, v)$. By ([15], Theorem 3.2), one has

$$
\operatorname{dim}\left(S_{M}^{\lambda} \cap \overline{\mathrm{Gr}}_{M}^{\mu}\right)=\left\langle\lambda+\mu, \check{\rho}_{M}\right\rangle, \operatorname{dim}\left(S^{\lambda} \cap \overline{\mathrm{Gr}}_{G}^{\mu^{\prime}}\right)=\left\langle\lambda+\mu^{\prime}, \check{\rho}\right\rangle,
$$

and these schemes are nonempty. So, the inclusion $S_{M}^{\lambda} \cap \overline{\mathrm{Gr}}_{M}^{\mu} \subset S^{\lambda} \cap \overline{\mathrm{Gr}}_{G}^{\mu^{\prime}}$ yields the desired inequality

$$
\left\langle\lambda+\mu, 2 \check{\rho}_{M}\right\rangle \leq\left\langle\lambda+\mu^{\prime}, 2 \check{\rho}\right\rangle
$$

Assume in addition $M$ antistandard. The inclusion

$$
S_{M}^{W_{0}^{M}(\mu)} \cap \overline{\mathrm{Gr}}_{M}^{\mu} \subset S^{W_{0}^{M}(\mu)} \cap \overline{\mathrm{Gr}}_{G}^{\mu^{\prime}}
$$

gives $\left\langle w_{0}^{M}(\mu)+\mu, 2 \check{\rho}_{M}\right\rangle \leq\left\langle w_{0}^{M}(\mu)+\mu^{\prime}, 2 \check{\rho}\right\rangle$, so $f(\mu) \leq\left\langle w_{0}^{M}(\mu), 2 \check{\rho}-2 \check{\rho}_{M}\right\rangle$. Since $M$ is antistandard,

$$
\left\langle\lambda-w_{0}^{M}(\mu), 2 \check{\rho}-2 \check{\rho}_{M}\right\rangle \geq 0,
$$

and this inequality is strict unless $\lambda \in-\Lambda_{M}^{+}$and $\mu=w_{0}^{M}(\lambda)$. Assume now $\lambda \in-\Lambda_{M}^{+}$and $\mu=w_{0}^{M}(\lambda)$. Since $w_{0}(\check{\rho})=-\check{\rho}$, the inequality (A2) becomes $0 \leq\left\langle-w_{0}(\lambda)+\mu^{\prime}, 2 \check{\rho}\right\rangle$, and it is strict unless $\lambda \in-\Lambda_{G}^{+}$and $\mu^{\prime}=w_{0}(\lambda)$. We are done.

\section{A.2 Application of the Casselman-Shalika formula}

\section{A.2.1}

Recall the following from ([3], 7.1). Let $\eta$ be a coweight of $G_{a d}$. A choice of an isomorphism $\epsilon_{\alpha}: \mathcal{O}(\langle\eta, \check{\alpha}\rangle) \widetilde{\rightarrow} \Omega$ for each simple root $\check{\alpha}$ of $G$ gives rise to a homomorphism $\chi: U(F) \rightarrow \mathbb{A}^{1}$ given as the sum over simple roots $\check{\alpha}$ of the maps

$$
U(F) \rightarrow U /[U, U](F) \stackrel{\check{\alpha}}{\rightarrow} F \stackrel{\epsilon_{\alpha}}{\rightarrow} \Omega(F) \stackrel{\text { Res }}{\rightarrow} \mathbb{A}^{1}
$$

The character $\chi$ is called admissible of conductor $\eta$.

Pick an admissible character $\chi_{-\lambda}: U(F) \rightarrow \mathbb{A}^{1}$ of conductor $-\operatorname{pr}(\lambda)$, here pr : $\Lambda \rightarrow \Lambda_{a d}$ is the projection, and $\Lambda_{a d}$ is the coweight lattice of $G_{a d}$. By ([3], Lemma 7.1.5) there is a map $\chi_{-\lambda}^{\lambda}: S^{\lambda} \rightarrow \mathbb{A}^{1}$, which is $\left(U(F), \chi_{-\lambda}\right)$-equivariant. The map $\chi_{-\lambda}^{\lambda}$ is unique up to an additive constant.

Let $i_{M}: S_{M}^{\lambda} \hookrightarrow S^{\lambda}$ be the natural inclusion.

Proposition A.2.2. Assume $M$ is antistandard. Let $\lambda \in \Lambda, v \in \Lambda^{+}$. The natural map

$$
\mathrm{R} \Gamma_{C}\left(S^{\lambda}, \mathcal{A}_{v} \otimes\left(\chi_{-\lambda}^{\lambda}\right)^{*} \mathcal{L}_{\psi}\right) \rightarrow \mathrm{R} \Gamma_{C}\left(S_{M}^{\lambda}, i_{M}^{*}\left(\mathcal{A}_{v} \otimes\left(\chi_{-\lambda}^{\lambda}\right)^{*} \mathcal{L}_{\psi}\right)\right)
$$


induces an isomorphism in the cohomological degree $\langle\lambda, 2 \check{\rho}\rangle$, and both complexes are placed in the cohomological degrees $\leq\langle\lambda, 2 \check{\rho}\rangle$.

Proof. By ([2], Theorem 1),

$$
\mathrm{R} \Gamma_{c}\left(S^{\lambda}, \mathcal{A}_{v} \otimes\left(\chi_{-\lambda}^{\lambda}\right)^{*} \mathcal{L}_{\psi}\right)
$$

is given by the Casselman-Shalika formula, namely, it vanishes unless $-\lambda \in \Lambda_{G}^{+}$and $\lambda=w_{0}(\nu)$. In the latter case, it identifies with $\overline{\mathbb{Q}}_{\ell}[-\langle\lambda, 2 \check{\rho}\rangle]$. More precisely, if $\lambda=w_{0}(\nu)$ then the natural map

$$
\left.\mathrm{R} \Gamma_{c}\left(S^{\lambda}, \mathcal{A}_{v} \otimes\left(\chi_{-\lambda}^{\lambda}\right)^{*} \mathcal{L}_{\psi}\right) \rightarrow i_{\lambda}^{*}\left(\mathcal{A}_{v} \otimes\left(\chi_{-\lambda}^{\lambda}\right)^{*} \mathcal{L}_{\psi}\right)\right) \underset{\rightarrow}{\rightarrow} \overline{\mathbb{Q}}_{\ell}[-\langle\lambda, 2 \check{\rho}\rangle]
$$

is an isomorphism, where $i_{\lambda}:$ Spec $k \rightarrow S^{\lambda}$ is the point $t^{\lambda}$.

Since $M$ is antistandard, $i_{M}^{*}\left(\chi_{-\lambda}^{\lambda}\right)^{*} \mathcal{L}_{\psi} \underset{\rightarrow}{\rightarrow} \overline{\mathbb{Q}}_{\ell}$. Our claim follows now from Proposition A.0.5.

\section{Acknowledgments}

I am very grateful to M. Finkelberg for numerous fruitful discussions on the subject. I also thank the referees for useful comments.

\section{References}

[1] Frenkel, E., D. Gaitsgory, D. Kazhdan, and K. Vilonen. "Geometric realization of Whittaker functions and the Langlands conjecture." J of AMS. 11, no. 2 (1998): 451-84.

[2] Frenkel, E., D. Gaitsgory, and K. Vilonen. "On the geometric Langlands conjecture." J. of AMS. 15, no. 2 (2001): 367-417.

[3] Frenkel, E., D. Gaitsgory, and K. Vilonen. "Whittaker patterns in the geometry of moduli spaces of bundles on curves." 153, no. 3 (2001): 699-748.

[4] Friedberg, S. and H. Jacquet. "Linear periods." J. Reine Angew. Math. 443 (1993): 91-139.

[5] Gaitsgory, D. "On a vanishing conjecture appearing in the geometric Langlands correspondence." Annals of Math. Second Series 160, no. 2 (2004): 617-82.

[6] D. Gaitsgory. The local and global versions of the Whittaker category. arXiv (1811): 02468.

[7] Jacquet, H., E. Lapid, and J. Rogawski. "Periods of automorphic forms, J. of AMS." 12, no. 1 (1999): 173-240.

[8] Jacquet, H. and J. Shalika. "Exterior square L-functions." Automorphic forms, Shimura varieties and L-functions (Ann Arbor proceedings 1988), vol. II, number 11 in Pespect. Math., 143-226. Academic Press, 1990.

[9] Laumon, G. "Correspondance de Langlands géométrique pour les corps de fonctions." Duke Math. J. 54, no. 2 (1987): 309-59.

[10] Lysenko, S. “Geometric Waldspurger periods." Compositio Math. 144, no. 2 (2008): 377-438. 
70 S. Lysenko

[11] S. Lysenko. "Local geometric Rankin-Selberg method for GL_n." Duke Math. J. 111, no. 3, (2002): 451-93.

[12] S. Lysenko. "Global geometrised Rankin-Selberg method for GL(n)." math.AG/0108208.

[13] S. Lysenko. “Geometric theta-lifting for the dual pair SO_2m, Sp_2n." Annales ENS, 4e serie, t.44 (2011): 427-93.

[14] S. Lysenko. "On the automorphic sheaves for GSp_4." arXiv:1901.04447.

[15] Mirkovic, I. and K. Vilonen. "Geometric Langlands duality and representations of algebraic groups over commutative rings." Ann. of Math. 166 (2007): 95-143.

[16] Okada, S. "Pieri Rules for Classical Groups and Equinumeration between Generalized Oscillating Tableaux and Semistandard Tableaux." The electronic journal of combinatorics 23, no. 4 (2016).

[17] Richardson, R. W. and T. A. Springer. "Combinatorics and geometry of $K$-orbits on the flag manifold." Linear algebraic groups and their representations (R. Elman, M. Schacher, V. Varadarajan, eds.) Contemporary Math, Vol. 153. 109-42. Providence: Amer. Math. Soc., 1993.

[18] Sakellaridis, Y. and A. Venkatesh. "Periods and Harmonic Analysis on Spherical Varieties." Astérisque 396 (2018). 